\title{
Direct $\beta$-Alkenylation of Ketones via Pd-Catalyzed Redox Cascade
}

\author{
Chengpeng Wang, Alexander J. Rago and Guangbin Dong* \\ Department of Chemistry, University of Chicago, Chicago, Illinois 60637, United States \\ *Email: gbdong@uchicago.edu
}

\section{Table of contents}

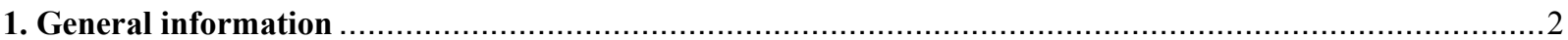

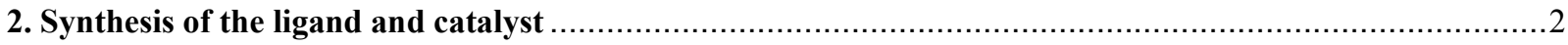

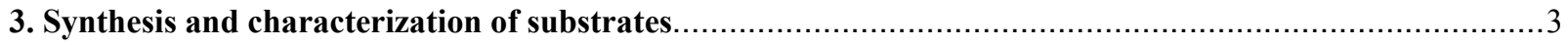

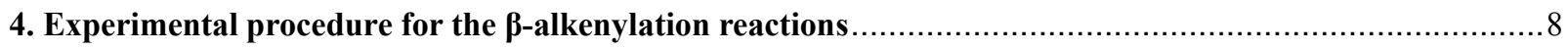

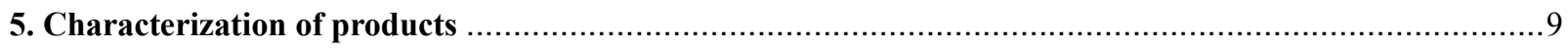

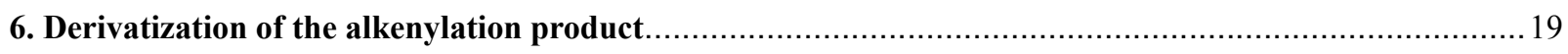

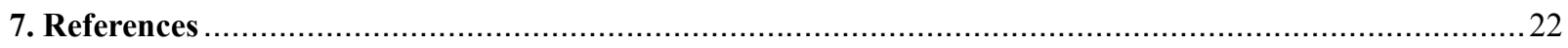

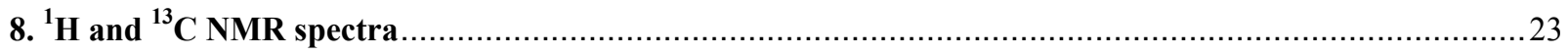




\section{General information}

Unless otherwise noted, all reactions were carried out in 8-mL culture tubes sealed with PTFE lined caps. Acetonitrile was distilled over calcium hydride and freeze-pump-thawed three times before use. 1,4-Dioxane was distilled over sodium and freeze-pump-thawed three times before use. $\mathrm{Pd}(\mathrm{MeCN})_{4}(\mathrm{OTf})_{2}$ and $\mathrm{P}(i-\mathrm{Pr})_{3}$ were synthesized based on modified literature procedures. Silver trifluoroacetate was purchased from Acros Organics. Potassium hydrogen phthalate was purchased from Sigma Aldrich. All commercially available substrates were used without further purification. Thin layer chromatography (TLC) analysis was run on silica gel plates purchased from EMD Chemical (silica gel 60, F254). Infrared spectra were recorded on a Nicolet iS5 FT-IR Spectrometer using neat thin film technique. High-resolution mass spectra (HRMS) were obtained on an Agilent 6224 Tof-MS spectrometer and are reported as $\mathrm{m} / \mathrm{z}$. Nuclear magnetic resonance spectra $\left({ }^{1} \mathrm{H} N \mathrm{NMR},{ }^{13} \mathrm{C}\right.$ NMR and ${ }^{19} \mathrm{~F}$ NMR $)$ were recorded with a Bruker Model DMX $400\left(400 \mathrm{MHz},{ }^{1} \mathrm{H}\right.$ at $400 \mathrm{MHz},{ }^{13} \mathrm{C}$ at $101 \mathrm{MHz},{ }^{19} \mathrm{~F}$ at $\left.376 \mathrm{MHz}\right)$. For $\mathrm{CDCl}_{3}$ solutions, the chemical shifts were reported as parts per million ( $\mathrm{ppm}$ ) referenced to residual protium or carbon of the solvents: $\mathrm{CHCl}_{3} \delta \mathrm{H}(7.26 \mathrm{ppm})$ and $\mathrm{CDCl}_{3} \delta \mathrm{C}(77.00 \mathrm{ppm})$. Coupling constants were reported in Hertz $(\mathrm{Hz})$. Data for ${ }^{1} \mathrm{H}$ NMR spectra were reported as following: chemical shift $(\delta, \mathrm{ppm})$, multiplicity $(\mathrm{br}=$ broad, $\mathrm{s}=$ singlet, $\mathrm{d}=$ doublet, $\mathrm{t}=$ triplet, $\mathrm{q}=$ quartet, $\mathrm{dd}=$ doublet of doublets, $\mathrm{td}=$ triplet of doublets, $\mathrm{ddd}=$ doublet of doublet of doublets, $\mathrm{m}=$ multiplet), coupling constant $(\mathrm{Hz})$, and integration.

\section{Synthesis of the ligand and catalyst}

$$
\mathrm{P}(i-\mathrm{Pr})_{3} \cdot \mathrm{HBF}_{4} \stackrel{\mathrm{NaH}, \mathrm{THF}}{\longrightarrow} \mathrm{P}(i-\mathrm{Pr})_{3}
$$

Triisopropylphosphine: The procedure was modified based on a literature report. ${ }^{1}$ A Schlenk flask was flame-dried and charged with triisopropylphosphonium tetrafluoroborate ${ }^{2}$ ( 1 equiv., $51.6 \mathrm{mmol}, 12.8 \mathrm{~g}$ ). The flask was transferred into the glovebox. Inside the glovebox, $\mathrm{NaH}$ (washed with hexane and dried before use, 1.2 equiv., $62 \mathrm{mmol}, 1.49 \mathrm{~g}$ ) and THF (distilled over sodium and freeze-pump-thawed three times before use, $155 \mathrm{~mL}$ ) were added, and the mixture was stirred at room temperature under $\mathrm{N}_{2}$ overnight. Inside the glovebox, the mixture was filtered through a short plug of basic alumina $(\sim 15 \mathrm{~g})$ and washed with small amount of THF. The filtrate was concentrated in vacuo inside the glovebox. After all the solvent was removed, the product was obtained as a colorless liquid in $94 \%$ yield $(7.8 \mathrm{~g})$. The product was stored in the glovebox.

$$
\mathrm{Pd}(\mathrm{OAC})_{2} \underset{\text { then } \mathrm{Et}_{2} \mathrm{O}}{\stackrel{\text { HOTf, } \mathrm{MeCN}}{\longrightarrow}} \mathrm{Pd}(\mathrm{MeCN})_{4}(\mathrm{OTf})_{2}
$$

Tetrakis(acetonitrile)palladium(II) bis(trifluoromethanesulfonate): A Schlenk flask was flame-dried and charged with $\operatorname{Pd}(\mathrm{OAc})_{2}$ (1 equiv., $6 \mathrm{mmol}, 1.35 \mathrm{~g}$ ). The flask was refilled with $\mathrm{N}_{2}$, and $\mathrm{MeCN}$ (distilled over calcium hydride before use, $40 \mathrm{~mL}$ ) was added via syringe. TfOH (3 equiv., $18 \mathrm{mmol}, 1.59 \mathrm{~mL}$ ) was dissolved in MeCN ( $5 \mathrm{~mL}$ ), and the solution was added slowly to the flask via syringe. The solution turned from dark red to light yellow during this process. After $10 \mathrm{~min}, \mathrm{Et}_{2} \mathrm{O}$ (distilled over sodium before use, $80 \mathrm{~mL}$ ) was added via syringe, and the mixture was further stirred for $2 \mathrm{~h}$. The mixture was filtered, washed with $\mathrm{Et}_{2} \mathrm{O}$ and dried under $\mathrm{N}_{2}$ flow. The solid was quickly collected and transferred into the glovebox. The product was obtained as a pale-yellow solid in $74 \%$ yield $(2.52 \mathrm{~g})$. The product was stored in the glovebox. 


\section{Synthesis and characterization of substrates}

Compounds $2 \mathbf{a}^{3}, \mathbf{2} \mathbf{b}^{3}, \mathbf{2} \mathbf{c}^{4}, \mathbf{2} \mathbf{d}^{5}, \mathbf{2} \mathbf{h}^{3}, \mathbf{2} \mathbf{i}^{3}, \mathbf{2} \mathbf{j}^{4}, \mathbf{2 \mathbf { l } ^ { 6 }}, \mathbf{2} \mathbf{m}^{6}, \mathbf{2} \mathbf{n}^{7}, \mathbf{2 \mathbf { o } ^ { 8 }}, \mathbf{2} \mathbf{u}^{9}$ and $\mathbf{1} \mathrm{e}^{10}$ were synthesized according to the literature procedures. Compounds $2 \mathrm{v}, 1 \mathrm{a}, 1 \mathrm{~b}, 1 \mathrm{c}, 1 \mathrm{~d}, 1 \mathrm{~h}$ and $1 \mathrm{i}$ were commercially available and used without further purification. Compounds $2 \mathbf{e}, \mathbf{2 f}, \mathbf{2 g}, \mathbf{2 k}, \mathbf{2 p}, \mathbf{2 q}, \mathbf{2 r}, \mathbf{2 s}, \mathbf{2 t}$, $\mathbf{1 f}$ and $\mathbf{1 g}$ were prepared according to the following procedures.

\section{Commercially available or known compounds:}<smiles>O=c1oc2ccccc2cc1Br</smiles>

$2 \mathbf{a}$<smiles>O=c1oc2ccc(Br)cc2cc1Br</smiles>

$2 h$<smiles>COc1ccc2cc(Br)c(=O)oc2c1</smiles>

$2 \mathbf{b}$<smiles>CC(=O)Oc1ccc2cc(Br)c(=O)oc2c1</smiles>

2c<smiles>O=c1oc2cc(O)ccc2cc1Br</smiles>

$2 \mathbf{j}$<smiles>O=c1oc2ccc([N+](=O)[O-])cc2cc1Br</smiles>

2d

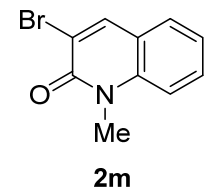

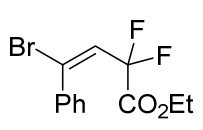

$2 n$

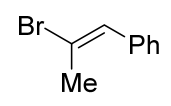

20

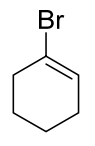

$2 u$<smiles>O=c1[nH]c2ccccc2cc1Br</smiles>

2I<smiles>O=C1CCCCC1</smiles>

$1 a$

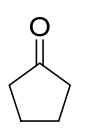

$1 b$<smiles>O=C1CCc2ccccc21</smiles>

$1 \mathrm{c}$

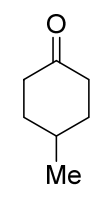

1d

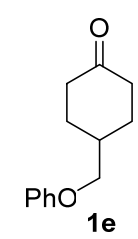

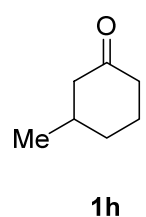

$1 \mathrm{~h}$

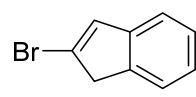

2v

\section{New compounds:}

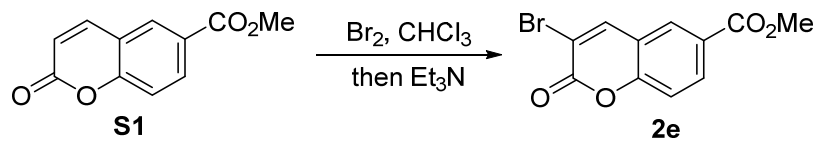

Methyl 3-bromo-2-oxo-2H-chromene-6-carboxylate (2e): A round bottom flask was charged with $\mathbf{S 1}{ }^{11}$ (1 equiv., $5 \mathrm{mmol}, 1.04 \mathrm{~g}$ ) and chloroform $(10 \mathrm{~mL})$, and the mixture was cooled in an ice bath. $\mathrm{Br}_{2}$ (1 equiv., $5 \mathrm{mmol}, 258 \mu \mathrm{L}$ ) was added dropwise, and the mixture was stirred at $50{ }^{\circ} \mathrm{C}$ for $6 \mathrm{~h} . \mathrm{Et}_{3} \mathrm{~N}$ ( 2 equiv., $10 \mathrm{mmol}, 1.39 \mathrm{~mL}$ ) was then added slowly, and the mixture was further stirred for $30 \mathrm{~min}$. The mixture was concentrated, and the residue was purified by column chromatography to give the product as a white solid in 67\% yield (949 mg). Melting point: 198 $-200{ }^{\circ} \mathrm{C} . \mathrm{R}_{f}=0.5$ (hexane/EtOAc $\left.=3: 1\right) .{ }^{1} \mathbf{H}$ NMR $\left(400 \mathrm{MHz}, \mathrm{CDCl}_{3}\right) \delta 8.23(\mathrm{dd}, J=8.7,2.0 \mathrm{~Hz}, 1 \mathrm{H}), 8.19(\mathrm{~d}, J=$ $2.0 \mathrm{~Hz}, 1 \mathrm{H}), 8.15(\mathrm{~s}, 1 \mathrm{H}), 7.40(\mathrm{~d}, J=8.7 \mathrm{~Hz}, 1 \mathrm{H}), 3.97(\mathrm{~s}, 3 \mathrm{H}) .{ }^{13} \mathbf{C}$ NMR $\left(101 \mathrm{MHz}, \mathrm{CDCl}_{3}\right) \delta 165.33,156.35$, 155.83, 143.92, 132.93, 129.11, 127.13, 119.09, 117.08, 112.89, 52.59. IR (KBr, $\left.\mathrm{cm}^{-1}\right) 3055,1742,1710,1616$, 1337, 767. HRMS calcd $\mathrm{C}_{11} \mathrm{H}_{8} \mathrm{BrO}_{4}[\mathrm{M}+\mathrm{H}]^{+}: 282.9600$. Found: 282.9595. 


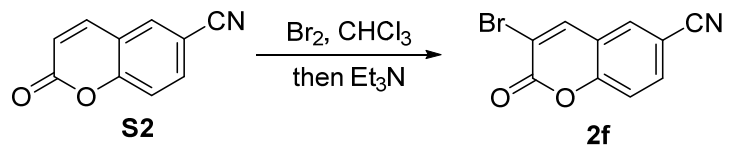

3-Bromo-2-0xo-2H-chromene-6-carbonitrile (2f): A round bottom flask was charged with $\mathbf{S 2}^{12}$ (1 equiv., 1.33 $\mathrm{mmol}, 228 \mathrm{mg}$ ) and chloroform $\left(2.67 \mathrm{~mL}\right.$ ), and the mixture was cooled in an ice bath. $\mathrm{Br}_{2}$ (1 equiv., $1.33 \mathrm{mmol}$, $68.6 \mu \mathrm{L}$ ) was added dropwise, and the mixture was stirred at $50{ }^{\circ} \mathrm{C}$ for $6 \mathrm{~h}^{-} \mathrm{Et}_{3} \mathrm{~N}$ (2 equiv., $2.67 \mathrm{mmol}, 370 \mu \mathrm{L}$ ) was then added slowly, and the mixture was further stirred for $30 \mathrm{~min}$. The mixture was concentrated, and the residue was purified by column chromatography to give the product as a white solid in $61 \%$ yield $(202 \mathrm{mg})$. Melting point: $233-236{ }^{\circ} \mathrm{C} . \mathrm{R}_{f}=0.5$ (hexane/EtOAc $\left.=3: 1\right) .{ }^{1} \mathbf{H}$ NMR $\left(400 \mathrm{MHz}, \mathrm{CDCl}_{3}\right) \delta 8.11(\mathrm{~s}, 1 \mathrm{H}), 7.87-7.77(\mathrm{~m}, 2 \mathrm{H})$, $7.46(\mathrm{dt}, J=8.3,0.7 \mathrm{~Hz}, 1 \mathrm{H}) .{ }^{13} \mathbf{C}$ NMR $\left(101 \mathrm{MHz}, \mathrm{CDCl}_{3}\right) \delta 155.56,155.26,142.51,134.79,131.38,119.88$, 118.29, 117.15, 114.48, 109.30. IR $\left(\mathrm{KBr}, \mathrm{cm}^{-1}\right)$ 3036, 2238, 1727, 1613, 1345, 848. HRMS calcd $\mathrm{C}_{10} \mathrm{H}_{5} \mathrm{BrNO}_{2}$ $[\mathrm{M}+\mathrm{H}]^{+}: 249.9498$. Found: 249.9494 .

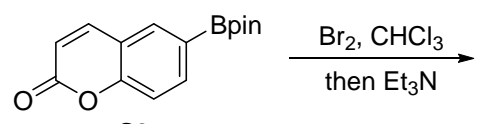

S3

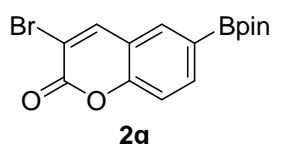

$2 \mathrm{~g}$

3-Bromo-6-(4,4,5,5-tetramethyl-1,3,2-dioxaborolan-2-yl)-2H-chromen-2-one (2g): A round bottom flask was charged with $\mathbf{S 3}^{13}$ (1 equiv., $1.0 \mathrm{mmol}, 272 \mathrm{mg}$ ) and chloroform $(2 \mathrm{~mL}$ ), and the mixture was cooled in an ice bath. $\mathrm{Br}_{2}(1$ equiv., $1.0 \mathrm{mmol}, 51.6 \mu \mathrm{L})$ was added dropwise, and the mixture was stirred at room temperature overnight. $\mathrm{Et}_{3} \mathrm{~N}$ ( 2 equiv., $2.0 \mathrm{mmol}, 278 \mu \mathrm{L}$ ) was then added slowly, and the mixture was further stirred for $30 \mathrm{~min}$. The mixture was concentrated, and the residue was purified by column chromatography to give the product as a white solid in $44 \%$ yield $(153 \mathrm{mg})$. Melting point: $179-181{ }^{\circ} \mathrm{C} . \mathrm{R}_{f}=0.6$ (hexane/EtOAc $\left.=3: 1\right) .{ }^{1} \mathbf{H}$ NMR $(400 \mathrm{MHz}$, $\left.\mathrm{CDCl}_{3}\right) \delta 8.09(\mathrm{~s}, 1 \mathrm{H}), 7.98(\mathrm{dd}, J=8.3,1.5 \mathrm{~Hz}, 1 \mathrm{H}), 7.91(\mathrm{~d}, J=1.5 \mathrm{~Hz}, 1 \mathrm{H}), 7.33(\mathrm{~d}, J=8.3 \mathrm{~Hz}, 1 \mathrm{H}), 1.36(\mathrm{~s}$, 12H). ${ }^{13} \mathbf{C}$ NMR $\left(101 \mathrm{MHz}, \mathrm{CDCl}_{3}\right) \delta 156.98,155.18,144.51,138.31,134.23,118.85,116.16,111.63,84.39,24.86$. IR $\left(\mathrm{KBr}, \mathrm{cm}^{-1}\right) 2975,1747,1613,1364,1143,965$. HRMS calcd $\mathrm{C}_{15} \mathrm{H}_{17} \mathrm{BBrO}_{4}[\mathrm{M}+\mathrm{H}]^{+}:$351.0398. Found: 351.0403 .

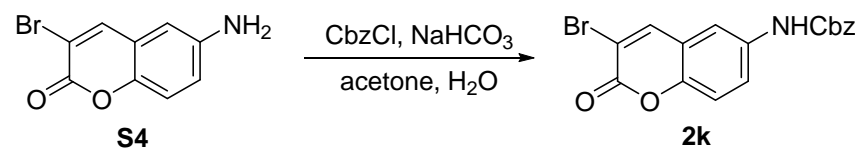

Benzyl (3-bromo-2-oxo-2H-chromen-6-yl)carbamate (2k): A round bottom flask was charged with $\mathbf{S 4} \mathbf{5}^{5}$ ( 1 equiv., $1.17 \mathrm{mmol}, 281 \mathrm{mg}), \mathrm{NaHCO}_{3}$ (2 equiv., $\left.2.34 \mathrm{mmol}, 197 \mathrm{mg}\right)$, water $(2.5 \mathrm{~mL})$ and acetone $(2.5 \mathrm{~mL}) . \mathrm{CbzCl}(1.1$ equiv., $1.29 \mathrm{mmol}, 184 \mu \mathrm{L}$ ) was then added dropwise, and the mixture was stirred at room temperature for $1 \mathrm{~h}$. The mixture was diluted with water and extracted with EtOAc. The combined organic layers were dried and concentrated, and the residue was purified by column chromatography to give the product as a pale-yellow solid in $86 \%$ yield $(377 \mathrm{mg})$. Melting point: $175-177^{\circ} \mathrm{C} . \mathrm{R}_{f}=0.3($ hexane/EtOAc $=3: 1) .{ }^{1} \mathbf{H}$ NMR $\left(400 \mathrm{MHz}\right.$, DMSO-d $\left.\mathrm{d}_{6}\right)$ $\delta 10.05(\mathrm{br}, 1 \mathrm{H}), 8.64(\mathrm{~s}, 1 \mathrm{H}), 7.87(\mathrm{~d}, J=2.6 \mathrm{~Hz}, 1 \mathrm{H}), 7.60$ (dd, $J=9.0,2.6 \mathrm{~Hz}, 1 \mathrm{H}), 7.47-7.30(\mathrm{~m}, 6 \mathrm{H}), 5.17$ (s, 2H). ${ }^{13}$ C NMR (101 MHz, DMSO-d $\left.\mathrm{d}_{6}\right) \delta 156.55,153.43,148.23,145.24,136.45,135.94,128.48,128.17,128.12$, $122.69,119.44,116.72,115.75,111.34,65.98$. IR $\left(\mathrm{KBr}, \mathrm{cm}^{-1}\right)$ 3336, 2923, 1720, 1700, 1567, 1217, 735. HRMS calcd $\mathrm{C}_{17} \mathrm{H}_{13} \mathrm{BrNO}_{4}[\mathrm{M}+\mathrm{H}]^{+}: 374.0022$. Found: 374.0025 .

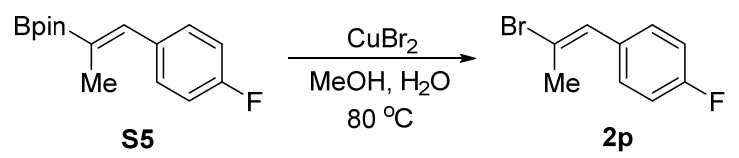


(E)-1-(2-Bromoprop-1-en-1-yl)-4-fluorobenzene (2p): A round bottom flask was equipped with a reflux condenser and charged with $\mathrm{CuBr}_{2}$ (3 equiv., $\left.21 \mathrm{mmol}, 4.69 \mathrm{~g}\right)$, water $(35 \mathrm{~mL})$, methanol $(35 \mathrm{~mL})$ and $\mathbf{S 5}^{14}$ (1 equiv., $7 \mathrm{mmol}, 1.84 \mathrm{~g}$ ). The mixture was heated at $80{ }^{\circ} \mathrm{C}$ for $4 \mathrm{~h}$. After cooling to room temperature, the mixture was extracted with $\mathrm{Et}_{2} \mathrm{O}$ four times. The combined organic layers were washed with water and brine, dried and concentrated. The residue was purified by column chromatography to give the product as a colorless oil in $72 \%$ yield (1.08 g). $E / Z>20: 1 . \mathrm{R}_{f}=0.6$ (hexane). ${ }^{1} \mathbf{H}$ NMR (400 MHz, $\left.\mathrm{CDCl}_{3}\right) \delta 7.21-7.13(\mathrm{~m}, 2 \mathrm{H}), 7.07-6.98(\mathrm{~m}$, 2H), $6.92(\mathrm{~s}, 1 \mathrm{H}), 2.43(\mathrm{~d}, J=1.4 \mathrm{~Hz}, 3 \mathrm{H}) .{ }^{13} \mathbf{C} \mathbf{N M R}\left(101 \mathrm{MHz}, \mathrm{CDCl}_{3}\right) \delta 161.74(\mathrm{~d}, J=247.3 \mathrm{~Hz}), 132.51(\mathrm{~d}, J=$ $3.5 \mathrm{~Hz}), 131.20,129.97$ (d, $J=8.1 \mathrm{~Hz}), 123.52$ (d, $J=1.7 \mathrm{~Hz}), 115.39$ (d, $J=21.5 \mathrm{~Hz}), 24.79 .{ }^{19} \mathbf{F ~ N M R}(376 \mathrm{MHz}$, $\left.\mathrm{CDCl}_{3}\right) \delta-114.33(\mathrm{tt}, J=8.6,5.4 \mathrm{~Hz}) . \mathbf{I R}\left(\mathrm{KBr}, \mathrm{cm}^{-1}\right) 2955,1601,1508,1226,1072,865$. HRMS calcd $\mathrm{C}_{9} \mathrm{H}_{8} \mathrm{BrF}$ $[\mathrm{M}]^{+}: 213.9788$. Found: 213.9789 .

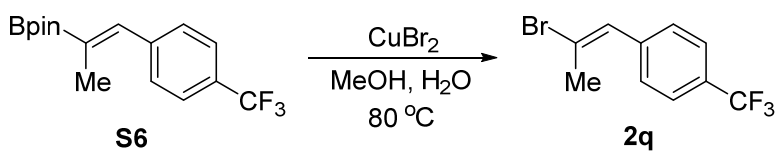

(E)-1-(2-Bromoprop-1-en-1-yl)-4-(trifluoromethyl)benzene (2q): A round bottom flask was equipped with a reflux condenser and charged with $\mathrm{CuBr}_{2}$ (3 equiv., $\left.26.1 \mathrm{mmol}, 5.83 \mathrm{~g}\right)$, water $(43.5 \mathrm{~mL})$, methanol (43.5 mL) and S6 ${ }^{15}$ (1 equiv., $8.7 \mathrm{mmol}, 2.71 \mathrm{~g}$ ). The mixture was heated at $80{ }^{\circ} \mathrm{C}$ for $4 \mathrm{~h}$. After cooling to room temperature, the mixture was extracted with $\mathrm{Et}_{2} \mathrm{O}$ four times. The combined organic layers were washed with water and brine, dried and concentrated. The residue was purified by column chromatography to give the product as a colorless oil in $63 \%$ yield. $E / Z>20: 1 . \mathrm{R}_{f}=0.5$ (hexane). ${ }^{1} \mathbf{H}$ NMR $\left(400 \mathrm{MHz}, \mathrm{CDCl}_{3}\right) \delta 7.60(\mathrm{~d}, J=8.1 \mathrm{~Hz}, 2 \mathrm{H}), 7.32(\mathrm{~d}, J=8.1 \mathrm{~Hz}$, $2 \mathrm{H}), 6.99(\mathrm{~s}, 1 \mathrm{H}), 2.46(\mathrm{~d}, J=1.5 \mathrm{~Hz}, 3 \mathrm{H}) .{ }^{13} \mathbf{C ~ N M R}\left(101 \mathrm{MHz}, \mathrm{CDCl}_{3}\right) \delta 139.92,131.07,129.18(\mathrm{q}, J=32.5 \mathrm{~Hz})$, $128.58,125.73,125.41(\mathrm{q}, J=3.8 \mathrm{~Hz}), 124.05(\mathrm{q}, J=272.0 \mathrm{~Hz}), 24.99 .{ }^{19} \mathbf{F} \mathbf{N M R}\left(376 \mathrm{MHz}, \mathrm{CDCl}_{3}\right) \delta-62.61 . \mathbf{I R}$ $\left(\mathrm{KBr}, \mathrm{cm}^{-1}\right)$ 2921, 1635, 1616, 1324, 1127, 1069, 869. HRMS calcd $\mathrm{C}_{10} \mathrm{H}_{8} \mathrm{BrF}_{3}[\mathrm{M}]^{+}:$: 263.9756. Found: 263.9750.

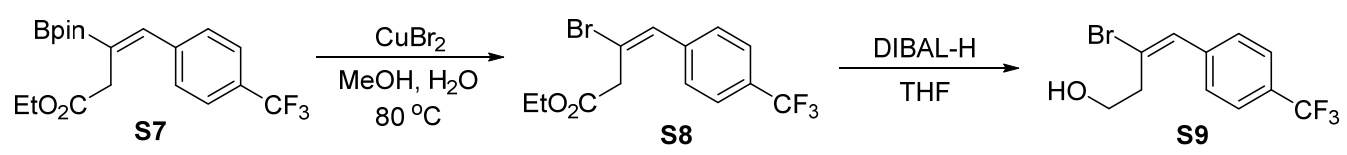

Ethyl (E)-3-bromo-4-(4-(trifluoromethyl)phenyl)but-3-enoate (S8): A round bottom flask was equipped with a reflux condenser and charged with $\mathrm{CuBr}_{2}$ (3 equiv., $\left.11.7 \mathrm{mmol}, 2.61 \mathrm{~g}\right)$, water $(20 \mathrm{~mL})$, methanol $(20 \mathrm{~mL})$ and $\mathbf{S} 7^{16}$ (1 equiv., $3.9 \mathrm{mmol}, 1.50 \mathrm{~g}$ ). The mixture was heated at $80{ }^{\circ} \mathrm{C}$ for $4 \mathrm{~h}$. After cooling to room temperature, the mixture was extracted with $\mathrm{Et}_{2} \mathrm{O}$ four times. The combined organic layers were washed with water and brine, dried and concentrated. The residue was purified by column chromatography to give the product as a colorless oil in $70 \%$ yield (920 mg). $E / Z>20: 1 . R_{f}=0.4$ (hexane/EtOAc $\left.=10: 1\right)$.

(E)-3-Bromo-4-(4-(trifluoromethyl)phenyl)but-3-en-1-ol (S9): A Schlenk flask was flame-dried and refilled with $\mathrm{N}_{2}$. S8 (1 equiv., $2.73 \mathrm{mmol}, 920 \mathrm{mg}$ ) was dissolved in THF (16 mL), and the solution was added to the flask via syringe. The mixture was cooled to $-78{ }^{\circ} \mathrm{C}$, and DIBAL-H (1 M in hexane, 2.4 equiv., $\left.6.55 \mathrm{mmol}, 6.55 \mathrm{~mL}\right)$ was added dropwise via syringe. The mixture was slowly warmed to $0{ }^{\circ} \mathrm{C}$ and stirred for $2 \mathrm{~h}$. Saturated sodium potassium tartrate solution was added, and the mixture was further stirred for $1 \mathrm{~h}$. The mixture was then extracted with EtOAc, and the combined organic layers were washed with water and brine, dried and concentrated. The residue was purified by column chromatography to give the product as a colorless oil in $96 \%$ yield $(773 \mathrm{mg})$. $\mathrm{R}_{f}=$ 0.8 (hexane/EtOAc = 1:1). ${ }^{1} \mathbf{H}$ NMR $\left(400 \mathrm{MHz} \mathrm{CDCl}_{3}\right) \delta 7.60(\mathrm{~d}, J=8.1 \mathrm{~Hz}, 2 \mathrm{H}), 7.46(\mathrm{~d}, J=7.7 \mathrm{~Hz}, 2 \mathrm{H}), 7.18(\mathrm{~s}$, $1 \mathrm{H}), 3.96(\mathrm{q}, J=5.9 \mathrm{~Hz}, 2 \mathrm{H}), 2.87(\mathrm{td}, J=6.0,0.8 \mathrm{~Hz}, 2 \mathrm{H}), 1.63(\mathrm{t}, J=5.7 \mathrm{~Hz}, 1 \mathrm{H}) .{ }^{13} \mathbf{C ~ N M R}\left(101 \mathrm{MHz}, \mathrm{CDCl}_{3}\right)$ $\delta 139.49,133.92,129.55(\mathrm{q}, J=32.6 \mathrm{~Hz}), 128.71,128.19,125.49$ (q, $J=3.8 \mathrm{~Hz}), 124.06(\mathrm{q}, J=272.0 \mathrm{~Hz}), 60.49$, 
39.11. ${ }^{19}$ F NMR $\left(376 \mathrm{MHz}, \mathrm{CDCl}_{3}\right) \delta$-62.64. IR $\left(\mathrm{KBr}, \mathrm{cm}^{-1}\right) 3343,2958,1617,1325,1126,1068,873$. HRMS calcd $\mathrm{C}_{11} \mathrm{H}_{11} \mathrm{BrF}_{3} \mathrm{O}[\mathrm{M}+\mathrm{H}]^{+}:$294.9940. Found: 294.9944.

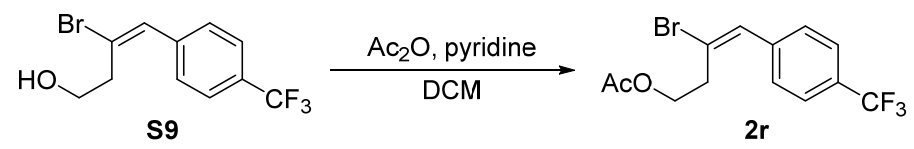

(E)-3-Bromo-4-(4-(trifluoromethyl)phenyl)but-3-en-1-yl acetate (2r): An 8-mL vial was charged with S9 (1 equiv., $0.5 \mathrm{mmol}, 147.5 \mathrm{mg}$ ), DCM $(2.5 \mathrm{~mL})$, pyridine (2.5 equiv., $1.25 \mathrm{mmol}, 100.6 \mu \mathrm{L})$ and $\mathrm{Ac}_{2} \mathrm{O}(1.5$ equiv., $0.75 \mathrm{mmol}, 70.7 \mu \mathrm{L}$ ), and the mixture was stirred at room temperature for $24 \mathrm{~h}$. The mixture was washed with $1 \mathrm{M}$ $\mathrm{HCl}$ twice, $\mathrm{NaHCO}_{3}$ and brine, dried and concentrated. The residue was purified by column chromatography to give the product as a colorless oil in $91 \%$ yield $(154 \mathrm{mg}) . \mathrm{R}_{f}=0.7$ (hexane/EtOAc $\left.=3: 1\right) .{ }^{1} \mathbf{H}$ NMR $(400 \mathrm{MHz}$, $\left.\mathrm{CDCl}_{3}\right) \delta 7.62(\mathrm{~d}, J=8.2 \mathrm{~Hz}, 2 \mathrm{H}), 7.43-7.35(\mathrm{~m}, 2 \mathrm{H}), 7.14(\mathrm{~s}, 1 \mathrm{H}), 4.35(\mathrm{t}, J=6.5 \mathrm{~Hz}, 2 \mathrm{H}), 2.94$ (td, $J=6.6,0.8$ $\mathrm{Hz}, 2 \mathrm{H}), 2.03(\mathrm{~s}, 3 \mathrm{H}) .{ }^{13} \mathbf{C}$ NMR $\left(101 \mathrm{MHz}, \mathrm{CDCl}_{3}\right) \delta 170.70,139.39(\mathrm{q}, J=1.5 \mathrm{~Hz}), 133.69,129.62(\mathrm{q}, J=32.7$ $\mathrm{Hz}), 128.44,126.69,125.56$ (q, $J=3.8 \mathrm{~Hz}), 123.97$ (q, $J=272.0 \mathrm{~Hz}), 61.89,35.64,20.80 .{ }^{19} \mathbf{F}$ NMR $(376 \mathrm{MHz}$, $\left.\mathrm{CDCl}_{3}\right) \delta$-62.66. IR $\left(\mathrm{KBr}, \mathrm{cm}^{-1}\right) 2960,1744,1616,1325,1127,874$. HRMS calcd $\mathrm{C}_{13} \mathrm{H}_{13} \mathrm{BrF}_{3} \mathrm{O}_{2}[\mathrm{M}+\mathrm{H}]^{+}$: 337.0046. Found: 337.0044 .

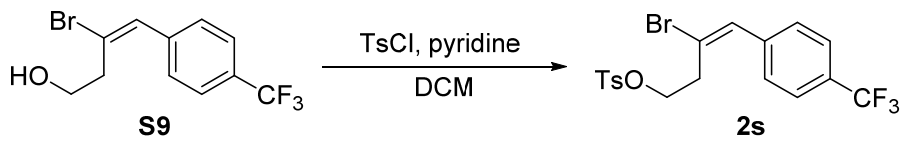

(E)-3-Bromo-4-(4-(trifluoromethyl)phenyl)but-3-en-1-yl 4-methylbenzenesulfonate (2s): A 4-mL vial was charged with $\mathbf{S 9}$ (1 equiv., $0.5 \mathrm{mmol}, 147.5 \mathrm{mg})$, DCM $(1 \mathrm{~mL})$, pyridine (2.2 equiv., $1.1 \mathrm{mmol}, 88.5 \mu \mathrm{L})$ and $\mathrm{TsCl}$ (1.1 equiv., $0.55 \mathrm{mmol}, 105 \mathrm{mg}$ ), and the mixture was stirred at room temperature overnight. The mixture was washed with $1 \mathrm{M} \mathrm{HCl}$ twice, $\mathrm{NaHCO}_{3}$ and brine, dried and concentrated. The residue was purified by column chromatography to give the product as a colorless oil in $75 \%$ yield $\left(168 \mathrm{mg}\right.$ ). $\mathrm{R}_{f}=0.5$ (hexane/EtOAc $\left.=5: 1\right) .{ }^{1} \mathbf{H}$ NMR $\left(400 \mathrm{MHz}, \mathrm{CDCl}_{3}\right) \delta 7.81-7.72(\mathrm{~m}, 2 \mathrm{H}), 7.59(\mathrm{~d}, J=8.2 \mathrm{~Hz}, 2 \mathrm{H}), 7.40-7.28(\mathrm{~m}, 4 \mathrm{H}), 7.11(\mathrm{~s}, 1 \mathrm{H}), 4.29$ (t, $J=6.3 \mathrm{~Hz}, 2 \mathrm{H}), 2.90(\mathrm{td}, J=6.2,0.9 \mathrm{~Hz}, 2 \mathrm{H}), 2.45(\mathrm{~s}, 3 \mathrm{H}) .{ }^{13} \mathbf{C} \mathbf{N M R}\left(101 \mathrm{MHz}, \mathrm{CDCl}_{3}\right) \delta 145.04,139.03(\mathrm{q}, J=$ $1.5 \mathrm{~Hz}), 134.54,132.66,129.87,129.71$ (q, $J=32.6 \mathrm{~Hz}), 128.49,127.93,125.56$ (q, $J=3.8 \mathrm{~Hz}), 124.95,123.95$ (q, $J=272.0 \mathrm{~Hz}), 67.11,35.83,21.61 .{ }^{19} \mathbf{F}$ NMR $\left(376 \mathrm{MHz}, \mathrm{CDCl}_{3}\right) \delta-62.64 . \mathbf{I R}\left(\mathrm{KBr}, \mathrm{cm}^{-1}\right) 2924,1364,1324,1177$, 1068, 901. HRMS calcd $\mathrm{C}_{18} \mathrm{H}_{17} \mathrm{BrF}_{3} \mathrm{O}_{3} \mathrm{~S}[\mathrm{M}+\mathrm{H}]^{+}:$449.0028. Found: 449.0023.

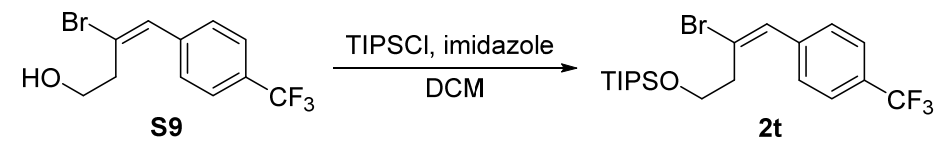

(E)-((3-Bromo-4-(4-(trifluoromethyl)phenyl)but-3-en-1-yl)oxy)triisopropylsilane (2t): A 4-mL vial was charged with S9 (1 equiv., $0.5 \mathrm{mmol}, 147.5 \mathrm{mg}$ ), DCM ( $1 \mathrm{~mL}$ ), imidazole (2 equiv., $1 \mathrm{mmol}, 68 \mathrm{mg}$ ) and TIPSCl (1 equiv., $0.5 \mathrm{mmol}, 107 \mu \mathrm{L}$ ), and the mixture was stirred at room temperature overnight. The mixture was washed with $1 \mathrm{M} \mathrm{HCl}$ twice, $\mathrm{NaHCO}_{3}$ and brine, dried and concentrated. The residue was purified by column chromatography to give the product as a colorless oil in $94 \%$ yield $(212 \mathrm{mg}) . \mathrm{R}_{f}=0.8($ hexane/EtOAc $=10: 1) .{ }^{1} \mathbf{H}$ NMR $\left(400 \mathrm{MHz}, \mathrm{CDCl}_{3}\right) \delta 7.59-7.53(\mathrm{~m}, 4 \mathrm{H}), 7.12(\mathrm{~s}, 1 \mathrm{H}), 4.03(\mathrm{t}, J=5.9 \mathrm{~Hz}, 2 \mathrm{H}), 2.85(\mathrm{td}, J=5.9,0.7 \mathrm{~Hz}, 2 \mathrm{H})$, $1.22-0.97(\mathrm{~m}, 21 \mathrm{H}) .{ }^{13} \mathbf{C}$ NMR $\left(101 \mathrm{MHz}, \mathrm{CDCl}_{3}\right) \delta 139.68,133.14,129.46,129.29(\mathrm{q}, J=32.5 \mathrm{~Hz}), 128.79$, 125.20 (q, $J=3.8 \mathrm{~Hz}), 124.14$ (q, $J=272.0 \mathrm{~Hz}), 61.08,39.75,17.96,11.95 .{ }^{19} \mathbf{F}$ NMR $\left(376 \mathrm{MHz}, \mathrm{CDCl}_{3}\right) \delta-62.56$. IR $\left(\mathrm{KBr}, \mathrm{cm}^{-1}\right)$ 2944, 2867, 1464, 1325, 1129, 882. HRMS calcd $\mathrm{C}_{20} \mathrm{H}_{31} \mathrm{BrF}_{3} \mathrm{OSi}[\mathrm{M}+\mathrm{H}]^{+}:$451.1274. Found: 451.1279 . 


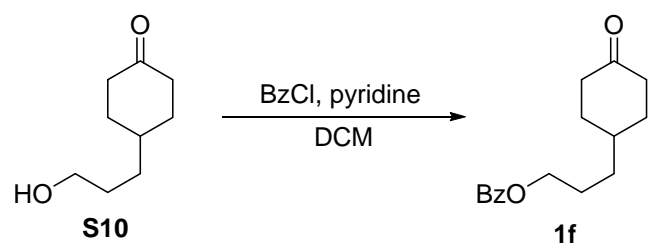

3-(4-Oxocyclohexyl)propyl benzoate (1f): A round bottom flask was charged with $\mathbf{S 1 0}{ }^{17}$ (1 equiv., $1.41 \mathrm{mmol}$, $220 \mathrm{mg}$ ), DCM $(7 \mathrm{~mL}$ ), pyridine (2.2 equiv., $3.10 \mathrm{mmol}, 250 \mu \mathrm{L})$ and $\mathrm{BzCl}$ (1.2 equiv., $1.69 \mathrm{mmol}, 196 \mu \mathrm{L}$ ), and the mixture was stirred at room temperature overnight. The mixture was washed with $1 \mathrm{M} \mathrm{HCl}$ twice, $\mathrm{NaHCO}_{3}$ and brine, dried and concentrated. The residue was purified by column chromatography to give the product as a white solid in $78 \%$ yield $\left(286 \mathrm{mg}\right.$ ). Melting point: $62-64{ }^{\circ} \mathrm{C} . \mathrm{R}_{f}=0.5$ (hexane/EtOAc $\left.=3: 1\right) .{ }^{1} \mathbf{H}$ NMR $(400 \mathrm{MHz}$, $\left.\mathrm{CDCl}_{3}\right) \delta 8.17-7.97(\mathrm{~m}, 2 \mathrm{H}), 7.63-7.54(\mathrm{~m}, 1 \mathrm{H}), 7.51-7.38(\mathrm{~m}, 2 \mathrm{H}), 4.35(\mathrm{t}, J=6.6 \mathrm{~Hz}, 2 \mathrm{H}), 2.49-2.26(\mathrm{~m}$, 4H), $2.16-1.99(\mathrm{~m}, 2 \mathrm{H}), 1.93-1.73(\mathrm{~m}, 3 \mathrm{H}), 1.54-1.36(\mathrm{~m}, 4 \mathrm{H}) .{ }^{13} \mathbf{C} \mathbf{N M R}\left(101 \mathrm{MHz}, \mathrm{CDCl}_{3}\right) \delta 211.99,166.58$, 132.90, 130.28, 129.47, 128.34, 64.94, 40.70, 35.70, 32.58, 31.89, 26.50. IR (KBr, cm $\left.{ }^{-1}\right) 2937,1711,1449,1283$, 1170, 716. HRMS calcd $\mathrm{C}_{16} \mathrm{H}_{21} \mathrm{O}_{3}[\mathrm{M}+\mathrm{H}]^{+}: 261.1485$. Found: 261.1483 .

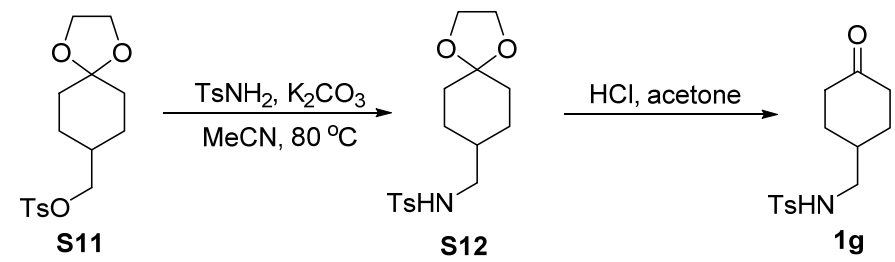

$N$-((1,4-Dioxaspiro[4.5]decan-8-yl)methyl)-4-methylbenzenesulfonamide (S12): A round bottom flask was equipped with a reflux condenser and charged with $\mathbf{S 1 1}{ }^{18}$ (1 equiv., $10 \mathrm{mmol}, 3.26 \mathrm{~g}$ ), $\mathrm{TsNH}_{2}$ (2 equiv., $20 \mathrm{mmol}$, $3.42 \mathrm{~g}$ ), $\mathrm{K}_{2} \mathrm{CO}_{3}$ (2 equiv., $20 \mathrm{mmol}, 2.76 \mathrm{~g}$ ) and $\mathrm{MeCN}\left(80 \mathrm{~mL}\right.$ ). The mixture was heated at $80{ }^{\circ} \mathrm{C}$ for $48 \mathrm{~h}$. The mixture was filtered, and the filtrate was concentrated. The residue was distributed between DCM and water, and the mixture was extracted with DCM. The combined organic layers were dried and concentrated, and the residue was purified by column chromatography to give the product as a colorless oil in $88 \%$ yield $(2.86 \mathrm{~g}) . \mathrm{R}_{f}=0.5$ $($ hexane/EtOAc $=1: 1)$.

4-Methyl-N-((4-oxocyclohexyl)methyl)benzenesulfonamide (1g): A round bottom flask was charged with S12 (1 equiv., $8.8 \mathrm{mmol}, 2.86 \mathrm{~g})$, acetone $(13.2 \mathrm{~mL})$ and $3 \mathrm{M} \mathrm{HCl}(13.2 \mathrm{~mL})$, and the mixture was stirred overnight. The acetone was removed in vacuo, and the residue was neutralized with $\mathrm{NaHCO}_{3}$ till $\mathrm{pH}=8$. The mixture was then extracted with DCM, dried and concentrated. The residue was purified by column chromatography to give the product as a white solid in $86 \%$ yield $\left(2.13 \mathrm{~g}\right.$ ). Melting point: $109-111{ }^{\circ} \mathrm{C} . \mathrm{R}_{f}=0.3$ (hexane/EtOAc $\left.=1: 1\right) .{ }^{1} \mathbf{H}$ NMR $\left(400 \mathrm{MHz}, \mathrm{CDCl}_{3}\right) \delta 7.85-7.67(\mathrm{~m}, 2 \mathrm{H}), 7.40-7.31(\mathrm{~m}, 2 \mathrm{H}), 5.01(\mathrm{t}, J=6.6 \mathrm{~Hz}, 1 \mathrm{H}), 2.88(\mathrm{t}, J=6.7 \mathrm{~Hz}$, $2 \mathrm{H}), 2.44(\mathrm{~s}, 3 \mathrm{H}), 2.42-2.31(\mathrm{~m}, 2 \mathrm{H}), 2.34-2.21(\mathrm{~m}, 2 \mathrm{H}), 2.12-1.99(\mathrm{~m}, 2 \mathrm{H}), 2.00-1.84(\mathrm{~m}, 1 \mathrm{H}), 1.45-1.26$ (m, 2H). ${ }^{13}$ C NMR (101 MHz, $\left.\mathrm{CDCl}_{3}\right) \delta 211.00,143.60,136.77,129.77,126.99,47.74,40.06,36.27,29.87,21.51$. IR $\left(\mathrm{KBr}, \mathrm{cm}^{-1}\right) 3278,2929,1712,1322,1159,1093,816$. HRMS calcd $\mathrm{C}_{14} \mathrm{H}_{20} \mathrm{NO}_{3} \mathrm{~S}[\mathrm{M}+\mathrm{H}]^{+}: 282.1158$. Found: 282.1155 . 


\section{Experimental procedure for the $\beta$-alkenylation reactions}

\section{General procedure:}

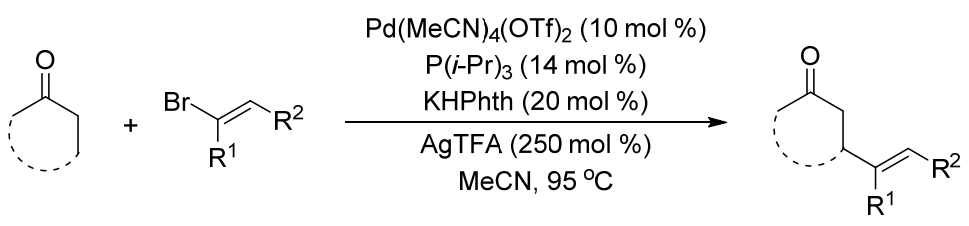

An 8-mL culture tube was flame-dried and charged with KHPhth (20 mol \%, 0.04 mmol, $8.2 \mathrm{mg})$ and AgTFA (2.5 equiv., $0.5 \mathrm{mmol}, 110.5 \mathrm{mg}$ ). The tube was then transferred into the glovebox. Inside the glovebox, $\mathrm{Pd}(\mathrm{MeCN})_{4}(\mathrm{OTf})_{2}(10 \mathrm{~mol} \%, 0.02 \mathrm{mmol}, 11.4 \mathrm{mg}), \mathrm{P}(\mathrm{i}-\mathrm{Pr})_{3}(14 \mathrm{~mol} \%, 0.028 \mathrm{mmol}, 5.3 \mu \mathrm{L}), \mathrm{MeCN}(\mathrm{distilled}$ over $\mathrm{CaH}_{2}$ and freeze-pump-thawed, $\left.0.8 \mathrm{~mL}\right)$, ketone $(2.5$ equiv., $0.5 \mathrm{mmol})$ and alkenyl bromide ( 1 equiv., 0.20 mmol) were added sequentially. The tube was sealed with a PTFE lined cap, transferred out of the glovebox and heated in an oil bath at $95{ }^{\circ} \mathrm{C}$ (oil temperature). After $18 \mathrm{~h}$, the mixture was allowed to cool to room temperature. The mixture was diluted with EtOAc, passed through a small plug of silica gel and eluted with EtOAc. The solvent was removed in vacuo, and the residue was purified by column chromatography to give the desired product.

Note: If the ketone or alkenyl bromide is a solid, it is weighed and added into the reaction tube before transferring into the glovebox.

\section{Representative procedure at 1 mmol scale:}

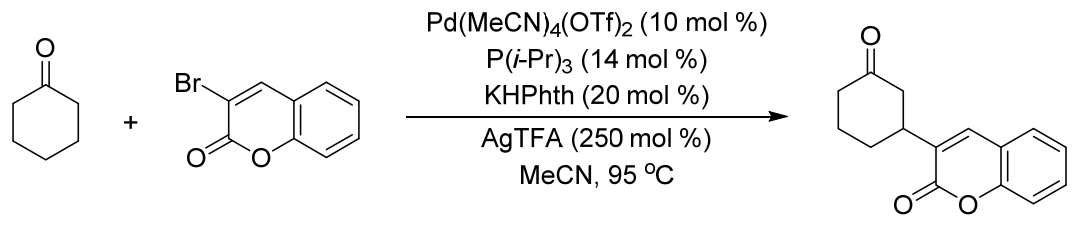

$1 \mathrm{a}$

$2 a$

3a

A $25-\mathrm{mL}$ Schlenk tube was

flame-dried and charged with KHPhth (20 mol \%, $0.2 \mathrm{mmol}, 40.8 \mathrm{mg})$, AgTFA (2.5 equiv., $2.5 \mathrm{mmol}, 553 \mathrm{mg})$ and 3-bromocoumarin ( 1 equiv., $1 \mathrm{mmol}, 225 \mathrm{mg}$ ). The tube was then transferred into the glovebox. Inside the glovebox, $\mathrm{Pd}(\mathrm{MeCN})_{4}(\mathrm{OTf})_{2}(10 \mathrm{~mol} \%, 0.1 \mathrm{mmol}, 56.9 \mathrm{mg}), \mathrm{P}(\mathrm{i}-\mathrm{Pr})_{3}(14 \mathrm{~mol} \%, 0.14 \mathrm{mmol}, 26.7 \mu \mathrm{L})$, MeCN (distilled over $\mathrm{CaH}_{2}$ and freeze-pump-thawed, $4 \mathrm{~mL}$ ) and cyclohexanone (2.5 equiv., $\left.2.5 \mathrm{mmol}, 259 \mu \mathrm{L}\right)$ were added sequentially. The tube was sealed with a septum, transferred out of the glovebox and heated in an oil bath at $95{ }^{\circ} \mathrm{C}$ (oil temperature). After $18 \mathrm{~h}$, the mixture was allowed to cool to room temperature. The mixture was diluted with EtOAc, passed through a small plug of silica gel and eluted with EtOAc. The solvent was removed in vacuo, and the residue was purified by column chromatography to give product 3a as a white solid in $73 \%$ yield (177.3 mg). 


\section{Characterization of products}

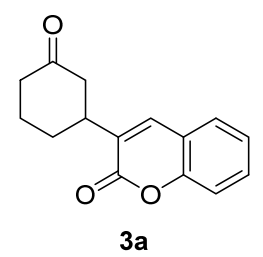

3-(3-Oxocyclohexyl)-2H-chromen-2-one (3a): Synthesized from 1a and 2a according to the general procedure. 77\% Yield $\left(37.2 \mathrm{mg}\right.$ ). White solid. Melting point: $120-122{ }^{\circ} \mathrm{C} . \mathrm{R}_{f}=0.6$ (hexane/EtOAc $\left.=1: 1\right) .{ }^{1} \mathbf{H} \mathbf{N M R}(400 \mathrm{MHz}$, $\left.\mathrm{CDCl}_{3}\right) \delta 7.54-7.43(\mathrm{~m}, 3 \mathrm{H}), 7.33(\mathrm{dd}, J=8.3,0.9 \mathrm{~Hz}, 1 \mathrm{H}), 7.30-7.25(\mathrm{~m}, 1 \mathrm{H}), 3.31-3.15(\mathrm{~m}, 1 \mathrm{H}), 2.75-2.59$ $(\mathrm{m}, 2 \mathrm{H}), 2.54-2.46(\mathrm{~m}, 1 \mathrm{H}), 2.45-2.32(\mathrm{~m}, 1 \mathrm{H}), 2.25-2.05(\mathrm{~m}, 2 \mathrm{H}), 2.00-1.74(\mathrm{~m}, 2 \mathrm{H}) .{ }^{13} \mathbf{C}$ NMR $(101 \mathrm{MHz}$, $\left.\mathrm{CDCl}_{3}\right) \delta 210.08,160.70,152.97,137.82,131.26,131.16,127.57,124.44,119.07,116.40,45.53,41.19,39.64$, 29.73, 24.79. IR $\left(\mathrm{KBr}, \mathrm{cm}^{-1}\right)$ 2951, 1711, 1610, 1456, 1176, 756. HRMS calcd $\mathrm{C}_{15} \mathrm{H}_{14} \mathrm{NaO}_{3}[\mathrm{M}+\mathrm{Na}]^{+}: 265.0835$. Found: 265.0838.

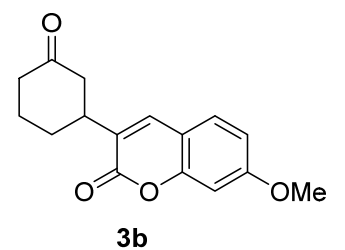

7-Methoxy-3-(3-oxocyclohexyl)-2H-chromen-2-one (3b): Synthesized from $\mathbf{1 a}$ and $\mathbf{2 b}$ according to the general procedure. $67 \%$ Yield $\left(36.3 \mathrm{mg}\right.$ ). Colorless oil. $\mathrm{R}_{f}=0.5$ (hexane/EtOAc $\left.=1: 1\right) .{ }^{1} \mathbf{H} \mathbf{~ N M R}\left(400 \mathrm{MHz}, \mathrm{CDCl}_{3}\right) \delta 7.44$ (s, 1H), 7.36 (d, $J=8.6 \mathrm{~Hz}, 1 \mathrm{H}), 6.85$ (dd, $J=8.6,2.5 \mathrm{~Hz}, 1 \mathrm{H}), 6.81$ (d, $J=2.4 \mathrm{~Hz}, 1 \mathrm{H}), 3.87$ (s, 3H), $3.45-3.06$ (m, 1H), $2.69-2.56(\mathrm{~m}, 2 \mathrm{H}), 2.53-2.44(\mathrm{~m}, 1 \mathrm{H}), 2.45-2.32(\mathrm{~m}, 1 \mathrm{H}), 2.18-2.03(\mathrm{~m}, 2 \mathrm{H}), 1.98-1.74(\mathrm{~m}, 2 \mathrm{H})$. ${ }^{13}$ C NMR $\left(101 \mathrm{MHz}, \mathrm{CDCl}_{3}\right) \delta 210.33,162.29,161.04,154.69,137.92,128.46,127.57,112.67,112.58,100.38$, 55.73, 45.65, 41.19, 39.57, 29.78, 24.79. IR $\left(\mathrm{KBr}, \mathrm{cm}^{-1}\right)$ 2939, 1712, 1616, 1508, 1241, 1154, 778. HRMS calcd $\mathrm{C}_{16} \mathrm{H}_{16} \mathrm{NaO}_{4}[\mathrm{M}+\mathrm{Na}]^{+}:$295.0941. Found: 295.0944.

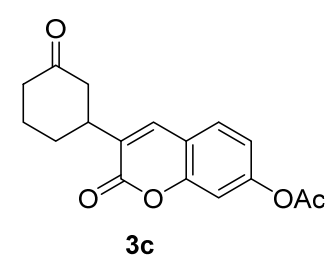

2-Oxo-3-(3-oxocyclohexyl)-2H-chromen-7-yl acetate (3c): Synthesized from 1a and 2c according to the general procedure. $73 \%$ Yield $\left(43.6 \mathrm{mg}\right.$ ). White solid. Melting point: $191-193{ }^{\circ} \mathrm{C} . \mathrm{R}_{f}=0.5$ (hexane/EtOAc $\left.=1: 1\right) .{ }^{1} \mathbf{H}$ NMR $\left(400 \mathrm{MHz}, \mathrm{CDCl}_{3}\right) \delta 7.56-7.44(\mathrm{~m}, 2 \mathrm{H}), 7.11(\mathrm{~d}, J=2.2 \mathrm{~Hz}, 1 \mathrm{H}), 7.05(\mathrm{dd}, J=8.5,2.2 \mathrm{~Hz}, 1 \mathrm{H}), 3.32-$ $3.16(\mathrm{~m}, 1 \mathrm{H}), 2.74-2.59(\mathrm{~m}, 2 \mathrm{H}), 2.54-2.45(\mathrm{~m}, 1 \mathrm{H}), 2.44-2.31(\mathrm{~m}, 4 \mathrm{H}), 2.19-2.04(\mathrm{~m}, 2 \mathrm{H}), 1.99-1.73(\mathrm{~m}$, 2H). ${ }^{13}$ C NMR $\left(101 \mathrm{MHz}, \mathrm{CDCl}_{3}\right) \delta 210.01,168.74,160.34,153.50,152.58,137.28,130.71,128.27,118.44$, 116.91, 109.99, 45.49, 41.19, 39.65, 29.69, 24.75, 21.10. IR (KBr, cm $\left.{ }^{-1}\right)$ 2929, 1759, 1707, 1429, 1208, 776. HRMS calcd $\mathrm{C}_{17} \mathrm{H}_{16} \mathrm{NaO}_{5}[\mathrm{M}+\mathrm{Na}]^{+}: 323.0890$. Found: 323.0897. 


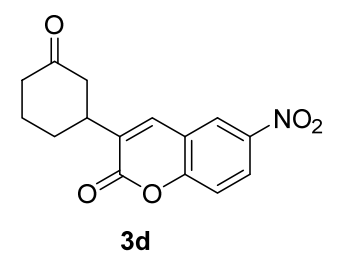

6-Nitro-3-(3-oxocyclohexyl)-2H-chromen-2-one (3d): Synthesized from 1a and $2 \mathrm{~d}$ according to the general procedure. $67 \%$ Yield $\left(38.5 \mathrm{mg}\right.$ ). Colorless oil. $\mathrm{R}_{f}=0.6$ (hexane/EtOAc $\left.=1: 1\right) .{ }^{1} \mathbf{H} \mathbf{N M R}\left(400 \mathrm{MHz}, \mathrm{CDCl}_{3}\right) \delta 8.44$ $(\mathrm{d}, J=2.6 \mathrm{~Hz}, 1 \mathrm{H}), 8.37(\mathrm{dd}, J=9.1,2.7 \mathrm{~Hz}, 1 \mathrm{H}), 7.60(\mathrm{~s}, 1 \mathrm{H}), 7.46(\mathrm{~d}, J=9.0 \mathrm{~Hz}, 1 \mathrm{H}), 3.49-3.17(\mathrm{~m}, 1 \mathrm{H}), 2.75$ $-2.58(\mathrm{~m}, 2 \mathrm{H}), 2.56-2.48(\mathrm{~m}, 1 \mathrm{H}), 2.48-2.35(\mathrm{~m}, 1 \mathrm{H}), 2.26-2.07(\mathrm{~m}, 2 \mathrm{H}), 2.01-1.76(\mathrm{~m}, 2 \mathrm{H}) .{ }^{13} \mathbf{C} \mathbf{~ N M R}(101$ $\left.\mathrm{MHz}, \mathrm{CDCl}_{3}\right) \delta 209.29,159.12,156.38,144.06,136.54,133.80,125.92,123.45,119.13,117.58,45.26,41.08$, 39.69, 29.56, 24.72. IR $\left(\mathrm{KBr}, \mathrm{cm}^{-1}\right)$ 2955, 1732, 1711, 1618, 1529, 1346, 764. HRMS calcd $\mathrm{C}_{15} \mathrm{H}_{13} \mathrm{NNaO}_{5}$ $[\mathrm{M}+\mathrm{Na}]^{+}: 310.0686$. Found: 310.0687 .

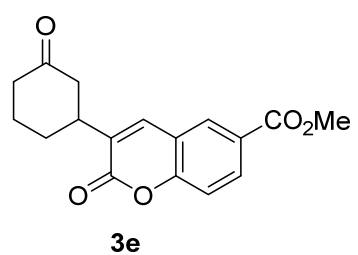

Methyl 2-oxo-3-(3-oxocyclohexyl)-2H-chromene-6-carboxylate (3e): Synthesized from 1a and 2e according to the general procedure. $75 \%$ Yield $\left(44.9 \mathrm{mg}\right.$ ). White solid. Melting point: $195-197{ }^{\circ} \mathrm{C} . \mathrm{R}_{f}=0.5$ (hexane/EtOAc $=$ 1:1). ${ }^{1}$ H NMR $\left(400 \mathrm{MHz}, \mathrm{CDCl}_{3}\right) \delta 8.21(\mathrm{~d}, J=2.0 \mathrm{~Hz}, 1 \mathrm{H}), 8.17(\mathrm{dd}, J=8.6,2.0 \mathrm{~Hz}, 1 \mathrm{H}), 7.54(\mathrm{~s}, 1 \mathrm{H}), 7.37(\mathrm{~d}, J$ $=8.6 \mathrm{~Hz}, 1 \mathrm{H}), 3.96(\mathrm{~s}, 3 \mathrm{H}), 3.49-3.08(\mathrm{~m}, 1 \mathrm{H}), 2.69-2.62(\mathrm{~m}, 2 \mathrm{H}), 2.58-2.45(\mathrm{~m}, 1 \mathrm{H}), 2.47-2.33(\mathrm{~m}, 1 \mathrm{H})$, $2.23-2.05(\mathrm{~m}, 2 \mathrm{H}), 2.01-1.74(\mathrm{~m}, 2 \mathrm{H}) .{ }^{13} \mathbf{C}$ NMR $\left(101 \mathrm{MHz}, \mathrm{CDCl}_{3}\right) \delta$ 209.75, 165.66, 160.01, 155.75, 137.44, $132.23,132.15,129.68,126.58,118.82,116.65,52.47,45.43,41.16,39.70,29.67,24.77$. IR $\left(\mathrm{KBr}, \mathrm{cm}^{-1}\right) 2951$, 1710, 1614, 1490, 1248, 766. HRMS calcd $\mathrm{C}_{17} \mathrm{H}_{16} \mathrm{NaO}_{5}[\mathrm{M}+\mathrm{Na}]^{+}: 323.0890$. Found: 323.0892.

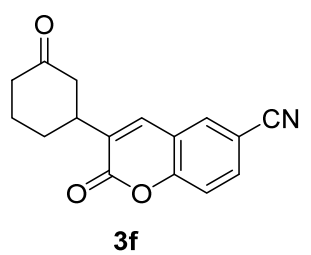

2-Oxo-3-(3-oxocyclohexyl)-2H-chromene-6-carbonitrile (3f): Synthesized from $1 \mathrm{a}$ and $2 \mathrm{f}$ according to the general procedure. $78 \%$ Yield $(41.6 \mathrm{mg})$. White solid. Melting point: $231-233{ }^{\circ} \mathrm{C} . \mathrm{R}_{f}=0.5$ (hexane/EtOAc $\left.=1: 1\right)$. ${ }^{1}$ H NMR $\left(400 \mathrm{MHz}, \mathrm{CDCl}_{3}\right) \delta 7.83(\mathrm{~d}, J=1.9 \mathrm{~Hz}, 1 \mathrm{H}), 7.76(\mathrm{dd}, J=8.6,2.0 \mathrm{~Hz}, 1 \mathrm{H}), 7.49(\mathrm{~s}, 1 \mathrm{H}), 7.43(\mathrm{~d}, J=8.6$ $\mathrm{Hz}, 1 \mathrm{H}), 3.34-3.13(\mathrm{~m}, 1 \mathrm{H}), 2.70-2.57(\mathrm{~m}, 2 \mathrm{H}), 2.55-2.47(\mathrm{~m}, 1 \mathrm{H}), 2.47-2.33(\mathrm{~m}, 1 \mathrm{H}), 2.22-2.06(\mathrm{~m}, 2 \mathrm{H})$, $1.99-1.76(\mathrm{~m}, 2 \mathrm{H}) .{ }^{13} \mathbf{C}$ NMR $\left(101 \mathrm{MHz}, \mathrm{CDCl}_{3}\right) \delta 209.35,159.19,155.17,136.10,134.06,133.68,132.01$, 119.72, 117.82, 117.54, 108.60, 45.27, 41.09, 39.71, 29.57, 24.72. IR (KBr, cm $\left.{ }^{-1}\right) 2933,2228,1729,1706,1489$, 1052, 748. HRMS calcd $\mathrm{C}_{16} \mathrm{H}_{13} \mathrm{NNaO}_{3}[\mathrm{M}+\mathrm{Na}]^{+}: 290.0788$. Found: 290.0786 .

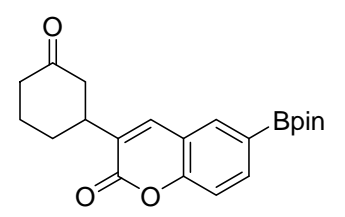

$3 g$ 
3-(3-Oxocyclohexyl)-6-(4,4,5,5-tetramethyl-1,3,2-dioxaborolan-2-yl)-2H-chromen-2-one (3g): Synthesized from $1 \mathrm{a}$ and $\mathbf{2 g}$ according to the general procedure. $62 \%$ Yield $(45.7 \mathrm{mg})$. Colorless oil. $\mathrm{R}_{f}=0.6$ (hexane/EtOAc $=$ 1:1). ${ }^{1} \mathbf{H}$ NMR $\left(400 \mathrm{MHz}, \mathrm{CDCl}_{3}\right) \delta 7.94(\mathrm{~d}, J=1.5 \mathrm{~Hz}, 1 \mathrm{H}), 7.91(\mathrm{dd}, J=8.2,1.5 \mathrm{~Hz}, 1 \mathrm{H}), 7.50(\mathrm{~s}, 1 \mathrm{H}), 7.30(\mathrm{~d}, J$ $=8.3 \mathrm{~Hz}, 1 \mathrm{H}), 3.36-3.11(\mathrm{~m}, 1 \mathrm{H}), 2.72-2.58(\mathrm{~m}, 2 \mathrm{H}), 2.55-2.31(\mathrm{~m}, 2 \mathrm{H}), 2.23-2.05(\mathrm{~m}, 2 \mathrm{H}), 1.97-1.73(\mathrm{~m}$, 2H), 1.36 (s, 12H). ${ }^{13} \mathbf{C}$ NMR (101 MHz, $\left.\mathrm{CDCl}_{3}\right) \delta 210.12,160.62,155.03,137.97,137.41,134.83,131.10,118.55$, 115.76, 84.23, 45.52, 41.19, 39.61, 29.68, 24.85, 24.74. IR (KBr, cm $\left.{ }^{-1}\right)$ 2978, 1717, 1606, 1365, 1145, 733. HRMS calcd $\mathrm{C}_{21} \mathrm{H}_{26} \mathrm{BO}_{5}[\mathrm{M}+\mathrm{H}]^{+}:$369.1868. Found: 369.1872 .

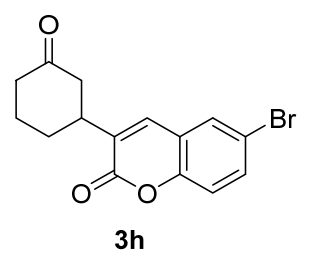

6-Bromo-3-(3-oxocyclohexyl)-2H-chromen-2-one (3h): Synthesized from $1 \mathbf{a}$ and $\mathbf{2 h}$ according to the general procedure. $63 \%$ Yield $\left(40.7 \mathrm{mg}\right.$ ). White solid. Melting point: $174-176{ }^{\circ} \mathrm{C} . \mathrm{R}_{f}=0.7$ (hexane/EtOAc $\left.=1: 1\right) .{ }^{1} \mathbf{H}$ NMR $\left(400 \mathrm{MHz}, \mathrm{CDCl}_{3}\right) \delta 7.62(\mathrm{~d}, J=2.3 \mathrm{~Hz}, 1 \mathrm{H}), 7.59(\mathrm{dd}, J=8.7,2.3 \mathrm{~Hz}, 1 \mathrm{H}), 7.41(\mathrm{~s}, 1 \mathrm{H}), 7.21(\mathrm{~d}, J=8.7 \mathrm{~Hz}$, $1 \mathrm{H}), 3.33-3.10(\mathrm{~m}, 1 \mathrm{H}), 2.67-2.56(\mathrm{~m}, 2 \mathrm{H}), 2.54-2.45(\mathrm{~m}, 1 \mathrm{H}), 2.46-2.29(\mathrm{~m}, 1 \mathrm{H}), 2.21-2.04(\mathrm{~m}, 2 \mathrm{H}), 1.98$ - 1.75 (m, 2H). ${ }^{13} \mathbf{C}$ NMR (101 MHz, $\left.\mathrm{CDCl}_{3}\right) \delta 209.74,160.02,151.81,136.49,133.92,132.58,129.89,120.61$, $118.15,116.99,45.41,41.15,39.69,29.66,24.75$. IR $\left(\mathrm{KBr}, \mathrm{cm}^{-1}\right) 2925,1713,1478,1246,1177,823$. HRMS calcd $\mathrm{C}_{15} \mathrm{H}_{14} \mathrm{BrO}_{3}[\mathrm{M}+\mathrm{H}]^{+}:$321.0121. Found: 321.0126 .

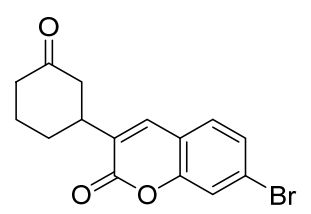

$3 \mathbf{i}$

7-Bromo-3-(3-oxocyclohexyl)-2H-chromen-2-one (3i): Synthesized from $\mathbf{1 a}$ and $\mathbf{2 i}$ according to the general procedure. $55 \%$ Yield $(41.8 \mathrm{mg})$. The arylation side product 3i' was isolated in $11 \%$ yield as an inseparable mixture with 3i $\left(\mathbf{3 i}: 3 \mathbf{i} \mathbf{i}^{\prime}=1: 0.19\right)$. Colorless oil. $\mathrm{R}_{f}=0.6$ (hexane/EtOAc $\left.=1: 1\right) .{ }^{\mathbf{1}} \mathbf{H} \mathbf{N M R}\left(400 \mathrm{MHz}, \mathrm{CDCl}_{3}\right) \delta 8.08(\mathrm{~s}$, $0.19 \mathrm{H}), 7.50(\mathrm{~d}, J=1.8 \mathrm{~Hz}, 1 \mathrm{H}), 7.45(\mathrm{~s}, 1 \mathrm{H}), 7.44-7.40(\mathrm{~m}, 1.19 \mathrm{H}), 7.33(\mathrm{~d}, J=8.2 \mathrm{~Hz}, 1 \mathrm{H}), 7.23-7.20$ (m, $0.19 \mathrm{H}), 7.20-7.16(\mathrm{~m}, 0.19 \mathrm{H}), 3.29-3.18(\mathrm{~m}, 1 \mathrm{H}), 3.17-3.05(\mathrm{~m}, 0.19 \mathrm{H}), 2.72-2.58(\mathrm{~m}, 2.19 \mathrm{H}), 2.54-2.44$ (m, 1.38H), $2.46-2.32(\mathrm{~m}, 1.19 \mathrm{H}), 2.26-2.06(\mathrm{~m}, 2.38 \mathrm{H}), 1.99-1.74(\mathrm{~m}, 2.38 \mathrm{H}) .{ }^{13} \mathbf{C}$ NMR $\left(101 \mathrm{MHz}, \mathrm{CDCl}_{3}\right)$ carbon signals are found at $\delta 209.85,209.60,159.93,157.06,153.53,153.17,149.34,144.03,137.14,131.59$, 128.51, 127.88, 127.35, 124.93, 123.68, 119.69, 117.99, 114.67, 111.34, 48.28, 45.40, 44.55, 41.17, 40.99, 39.70, 32.28, 29.61, 25.27, 24.73. IR $\left(\mathrm{KBr}, \mathrm{cm}^{-1}\right)$ 2942, 1737, 1714, 1599, 1055, 731. HRMS calcd $\mathrm{C}_{15} \mathrm{H}_{14} \mathrm{BrO}_{3}[\mathrm{M}+\mathrm{H}]^{+}$: 321.0121. Found: 321.0130 .

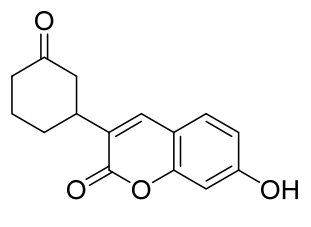

3j

7-Hydroxy-3-(3-oxocyclohexyl)-2H-chromen-2-one (3j): Synthesized from $\mathbf{1 a}$ and $\mathbf{2} \mathbf{j}$ according to the general 
procedure. $72 \%$ Yield $(37.3 \mathrm{mg})$. White solid. Melting point: $180-181{ }^{\circ} \mathrm{C} . \mathrm{R}_{f}=0.4$ (hexane/EtOAc $\left.=1: 1\right) .{ }^{1} \mathbf{H}$ NMR (400 MHz, Acetone-d $\left.\mathrm{d}_{6}\right) \delta 9.37(\mathrm{br}, 1 \mathrm{H}), 7.73(\mathrm{~s}, 1 \mathrm{H}), 7.49(\mathrm{~d}, J=8.5 \mathrm{~Hz}, 1 \mathrm{H}), 6.84(\mathrm{dd}, J=8.5,2.3 \mathrm{~Hz}, 1 \mathrm{H})$, 6.75 (d, $J=2.3 \mathrm{~Hz}, 1 \mathrm{H}$ ), 3.08 (ddddd, $J=12.3,11.2,4.3,3.3,0.9 \mathrm{~Hz}, 1 \mathrm{H}), 2.62$ (ddd, $J=13.5,12.4,1.0 \mathrm{~Hz}, 1 \mathrm{H}$ ), 2.50 (ddt, $J=13.7,4.1,2.0 \mathrm{~Hz}, 1 \mathrm{H}), 2.42$ (dddd, $J=13.9,12.7,6.1,1.0 \mathrm{~Hz}, 1 \mathrm{H}$ ), 2.31 (dddt, $J=14.3,4.7,3.3,1.7$ $\mathrm{Hz}, 1 \mathrm{H}), 2.18-2.07(\mathrm{~m}, 2 \mathrm{H}), 1.99-1.84(\mathrm{~m}, 1 \mathrm{H}), 1.83-1.67(\mathrm{~m}, 1 \mathrm{H}) .{ }^{13} \mathbf{C}$ NMR $\left(101 \mathrm{MHz}\right.$, Acetone-d $\left.\mathrm{d}_{6}\right) \delta 209.42$, $161.41,161.29,155.66,138.79,130.11,128.05,113.77,113.19,102.83,46.47,41.51,40.10,30.56,25.83$. IR $(\mathrm{KBr}$, $\left.\mathrm{cm}^{-1}\right) 3228,2950,1701,1612,1579,1258,1152,849$. HRMS calcd $\mathrm{C}_{15} \mathrm{H}_{15} \mathrm{O}_{4}[\mathrm{M}+\mathrm{H}]^{+}: 259.0965$. Found: 259.0970 .

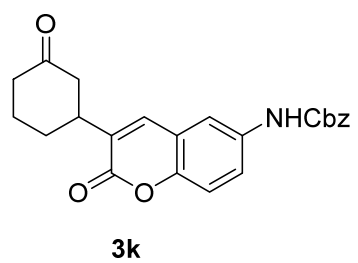

Benzyl (2-oxo-3-(3-0xocyclohexyl)-2H-chromen-6-yl)carbamate (3k): Synthesized from $1 \mathrm{a}$ and $\mathbf{2 k}$ according to the general procedure. 76\% Yield (59.6 mg). Pale-yellow oil. $\mathrm{R}_{f}=0.5$ (hexane/EtOAc $\left.=1: 1\right) .{ }^{1} \mathbf{H} ~ \mathbf{N M R}(400 \mathrm{MHz}$, $\left.\mathrm{CDCl}_{3}\right) \delta 7.77$ (br, 1H), 7.44 (s, 1H), $7.42-7.32(\mathrm{~m}, 6 \mathrm{H}), 7.23$ (d, J=8.9 Hz, 1H), 7.08 (br, 1H), $5.21(\mathrm{~s}, 2 \mathrm{H}), 3.31$ $-3.09(\mathrm{~m}, 1 \mathrm{H}), 2.70-2.53(\mathrm{~m}, 2 \mathrm{H}), 2.52-2.43(\mathrm{~m}, 1 \mathrm{H}), 2.44-2.31(\mathrm{~m}, 1 \mathrm{H}), 2.18-2.02(\mathrm{~m}, 2 \mathrm{H}), 1.96-1.75(\mathrm{~m}$, 2H). ${ }^{13}$ C NMR (101 MHz, $\left.\mathrm{CDCl}_{3}\right) \delta 210.20,160.68,153.38,148.92,137.64,135.72,134.43,131.78,128.61$, 128.45, 128.31, 121.93, 119.33, 116.81, 116.59, 67.24, 45.50, 41.11, 39.57, 29.71, 24.80. IR (KBr, $\left.\mathrm{cm}^{-1}\right) 3313$, 2952, 1708, 1551, 1221, 1057, 733. HRMS calcd $\mathrm{C}_{23} \mathrm{H}_{21} \mathrm{NNaO}_{5}[\mathrm{M}+\mathrm{Na}]^{+}:$414.1312. Found: 414.1310 .

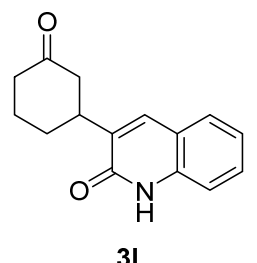

3-(3-Oxocyclohexyl)quinolin-2(1H)-one (3I): Synthesized from 1a and 21 according to the general procedure. 53\% Yield $(25.7 \mathrm{mg})$. White solid. Melting point: $254-257^{\circ} \mathrm{C} . \mathrm{R}_{f}=0.2($ hexane/EtOAc $=1: 1) .{ }^{1} \mathbf{H}$ NMR $(400 \mathrm{MHz}$, $\left.\mathrm{CDCl}_{3}\right) \delta 11.57(\mathrm{~s}, 1 \mathrm{H}), 7.61(\mathrm{~s}, 1 \mathrm{H}), 7.55(\mathrm{dd}, J=7.8,1.3 \mathrm{~Hz}, 1 \mathrm{H}), 7.49(\mathrm{ddd}, J=8.5,7.2,1.4 \mathrm{~Hz}, 1 \mathrm{H}), 7.34(\mathrm{dd}, J$ $=8.2,1.0 \mathrm{~Hz}, 1 \mathrm{H}), 7.22(\mathrm{ddd}, J=8.1,7.3,1.1 \mathrm{~Hz}, 1 \mathrm{H}), 3.58-3.39(\mathrm{~m}, 1 \mathrm{H}), 2.77-2.63(\mathrm{~m}, 2 \mathrm{H}), 2.58-2.38(\mathrm{~m}$, $2 \mathrm{H}), 2.24-2.08(\mathrm{~m}, 2 \mathrm{H}), 2.01-1.82(\mathrm{~m}, 2 \mathrm{H}) .{ }^{13} \mathbf{C} \mathbf{~ N M R}\left(101 \mathrm{MHz}, \mathrm{CDCl}_{3}\right) \delta 210.99,163.15,137.28,135.55$, 135.52, 130.04, 127.51, 122.66, 119.90, 115.38, 46.17, 41.37, 38.70, 30.20, 25.05. IR (KBr, cm $\left.{ }^{-1}\right) 3157,2948$, 1709, 1652, 1568, 1436, 668. HRMS calcd $\mathrm{C}_{15} \mathrm{H}_{16} \mathrm{NO}_{2}[\mathrm{M}+\mathrm{H}]^{+}: 242.1176$. Found: 242.1177.

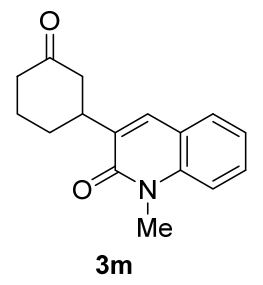

1-Methyl-3-(3-oxocyclohexyl)quinolin-2(1H)-one (3m): Synthesized from $1 \mathrm{a}$ and $\mathbf{2 m}$ according to the general 
procedure. $58 \%$ Yield $(29.7 \mathrm{mg})$. White solid. Melting point: $138-140{ }^{\circ} \mathrm{C} . \mathrm{R}_{f}=0.4$ (hexane/EtOAc $\left.=1: 1\right) .{ }^{1} \mathbf{H}$ NMR $\left(400 \mathrm{MHz}, \mathrm{CDCl}_{3}\right) \delta 7.59-7.50(\mathrm{~m}, 2 \mathrm{H}), 7.50(\mathrm{~s}, 1 \mathrm{H}), 7.39-7.32(\mathrm{~m}, 1 \mathrm{H}), 7.29-7.20(\mathrm{~m}, 1 \mathrm{H}), 3.75(\mathrm{~s}$, $3 \mathrm{H}), 3.49-3.33(\mathrm{~m}, 1 \mathrm{H}), 2.77-2.58(\mathrm{~m}, 2 \mathrm{H}), 2.54-2.33(\mathrm{~m}, 2 \mathrm{H}), 2.22-2.03(\mathrm{~m}, 2 \mathrm{H}), 1.97-1.77(\mathrm{~m}, 2 \mathrm{H}) .{ }^{13} \mathbf{C}$ NMR $\left(101 \mathrm{MHz}, \mathrm{CDCl}_{3}\right) \delta 211.01,161.56,138.93,135.32,133.92,129.96,128.43,122.15,120.27,113.86,46.00$, 41.34, 39.43, 30.21, 29.72, 24.99. IR $\left(\mathrm{KBr}, \mathrm{cm}^{-1}\right)$ 2927, 1705, 1641, 1593, 1459, 1224, 783. HRMS calcd $\mathrm{C}_{16} \mathrm{H}_{18} \mathrm{NO}_{2}[\mathrm{M}+\mathrm{H}]^{+}: 256.1332$. Found: 256.1341 .

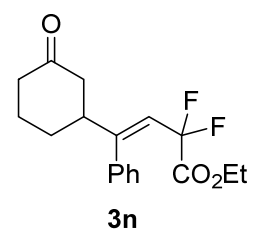

Ethyl (Z)-2,2-difluoro-4-(3-oxocyclohexyl)-4-phenylbut-3-enoate (3n): Synthesized from 1a and $2 \mathrm{n}$ according to the general procedure except for using 1,4-dioxane $(0.8 \mathrm{~mL})$ as the solvent at $90{ }^{\circ} \mathrm{C} .46 \%$ Yield $(29.6 \mathrm{mg})$. Light-yellow oil. $\mathrm{R}_{f}=0.3$ (hexane/EtOAc = 3:1). ${ }^{1} \mathbf{H}$ NMR $\left(400 \mathrm{MHz}, \mathrm{CDCl}_{3}\right) \delta 7.36-7.30(\mathrm{~m}, 3 \mathrm{H}), 7.09-7.01$ $(\mathrm{m}, 2 \mathrm{H}), 5.78(\mathrm{td}, J=11.3,1.2 \mathrm{~Hz}, 1 \mathrm{H}), 3.89(\mathrm{q}, J=7.2 \mathrm{~Hz}, 2 \mathrm{H}), 2.86-2.65(\mathrm{~m}, 1 \mathrm{H}), 2.47$ (ddt, $J=13.8,4.0,2.0$ $\mathrm{Hz}, 1 \mathrm{H}), 2.43-2.32(\mathrm{~m}, 1 \mathrm{H}), 2.30-2.19(\mathrm{~m}, 2 \mathrm{H}), 2.16-2.03(\mathrm{~m}, 1 \mathrm{H}), 2.02-1.91(\mathrm{~m}, 1 \mathrm{H}), 1.79-1.47(\mathrm{~m}, 2 \mathrm{H})$, $1.16(\mathrm{t}, J=7.2 \mathrm{~Hz}, 3 \mathrm{H}) .{ }^{13} \mathbf{C}$ NMR $\left(101 \mathrm{MHz}, \mathrm{CDCl}_{3}\right) \delta 209.86,163.37(\mathrm{t}, J=33.8 \mathrm{~Hz}), 154.13(\mathrm{t}, J=9.0 \mathrm{~Hz})$, 136.21, 128.38 (t, $J=1.9 \mathrm{~Hz}), 128.26,127.96,119.52(\mathrm{t}, J=28.3 \mathrm{~Hz}), 112.28(\mathrm{t}, J=246.1 \mathrm{~Hz}), 62.69,47.04,46.03$, 40.98, 29.62, 24.78, 13.63. ${ }^{19}$ F NMR $\left(376 \mathrm{MHz}, \mathrm{CDCl}_{3}\right) \delta-92.28$ (dd, $\left.J=277.3,10.8 \mathrm{~Hz}\right),-92.50$ (dd, $J=277.3$, $11.2 \mathrm{~Hz})$. IR $\left(\mathrm{KBr}, \mathrm{cm}^{-1}\right) 2942,1771,1713,1306,1195,1097,704$. HRMS calcd $\mathrm{C}_{18} \mathrm{H}_{21} \mathrm{~F}_{2} \mathrm{O}_{3}[\mathrm{M}+\mathrm{H}]^{+}: 323.1453$. Found: 323.1461 .

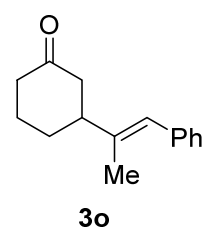

(E)-3-(1-Phenylprop-1-en-2-yl)cyclohexan-1-one (3o) ${ }^{19}$ : Synthesized from 1a and 20 according to the general procedure except for using 1,4-dioxane $(0.8 \mathrm{~mL})$ as the solvent at $90{ }^{\circ} \mathrm{C} .53 \%$ Yield $(22.6 \mathrm{mg})$. Colorless oil. $\mathrm{R}_{f}=$ 0.4 (hexane/EtOAc = 5:1). ${ }^{1} \mathbf{H}$ NMR $\left(400 \mathrm{MHz}, \mathrm{CDCl}_{3}\right) \delta 7.37-7.28(\mathrm{~m}, 2 \mathrm{H}), 7.25-7.16(\mathrm{~m}, 3 \mathrm{H}), 6.31(\mathrm{~s}, 1 \mathrm{H})$, $2.64-2.38(\mathrm{~m}, 4 \mathrm{H}), 2.37-2.24(\mathrm{~m}, 1 \mathrm{H}), 2.18-2.07(\mathrm{~m}, 1 \mathrm{H}), 2.05-1.95(\mathrm{~m}, 1 \mathrm{H}), 1.86(\mathrm{~d}, J=1.3 \mathrm{~Hz}, 3 \mathrm{H}), 1.81-$ $1.66(\mathrm{~m}, 2 \mathrm{H}) .{ }^{13} \mathrm{C}$ NMR $\left(101 \mathrm{MHz}, \mathrm{CDCl}_{3}\right) \delta 211.41,140.30,137.91,128.88,128.03,126.19,124.79,48.28,46.74$, $41.25,30.08,25.25,15.71$.

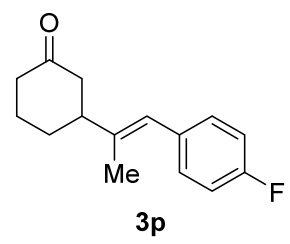

(E)-3-(1-(4-Fluorophenyl)prop-1-en-2-yl)cyclohexan-1-one (3p): Synthesized from 1a and $2 p$ according to the general procedure except for using 1,4-dioxane $(0.8 \mathrm{~mL})$ as the solvent at $90{ }^{\circ} \mathrm{C} .57 \%$ Yield $(26.4 \mathrm{mg})$. Colorless oil. $\mathrm{R}_{f}=0.4$ (hexane/EtOAc = 5:1). ${ }^{1} \mathbf{H}$ NMR $\left(400 \mathrm{MHz}, \mathrm{CDCl}_{3}\right) \delta 7.22-7.12(\mathrm{~m}, 2 \mathrm{H}), 7.07-6.96(\mathrm{~m}, 2 \mathrm{H}), 6.26(\mathrm{~s}$, $1 \mathrm{H}), 2.61-2.38(\mathrm{~m}, 4 \mathrm{H}), 2.36-2.25(\mathrm{~m}, 1 \mathrm{H}), 2.22-2.06(\mathrm{~m}, 1 \mathrm{H}), 2.03-1.91(\mathrm{~m}, 1 \mathrm{H}), 1.83(\mathrm{~d}, J=1.3 \mathrm{~Hz}, 3 \mathrm{H})$, 
$1.78-1.64(\mathrm{~m}, 2 \mathrm{H}) .{ }^{13} \mathbf{C}$ NMR $\left(101 \mathrm{MHz}, \mathrm{CDCl}_{3}\right) \delta 211.21,161.17(\mathrm{~d}, J=245.5 \mathrm{~Hz}), 140.27(\mathrm{~d}, J=1.3 \mathrm{~Hz})$, 133.85 (d, $J=3.3 \mathrm{~Hz}), 130.36$ (d, $J=7.8 \mathrm{~Hz}), 123.69,114.83$ (d, $J=21.3 \mathrm{~Hz}), 48.13,46.64,41.18,29.99,25.16$, 15.56. ${ }^{19}$ F NMR $\left(376 \mathrm{MHz}, \mathrm{CDCl}_{3}\right) \delta-116.10(\mathrm{tt}, J=8.5,5.5 \mathrm{~Hz})$. IR $\left(\mathrm{KBr}, \mathrm{cm}^{-1}\right) 2940,1714,1600,1508,1225$, 866. HRMS calcd $\mathrm{C}_{15} \mathrm{H}_{18} \mathrm{FO}[\mathrm{M}+\mathrm{H}]^{+}:$233.1336. Found: 233.1344.

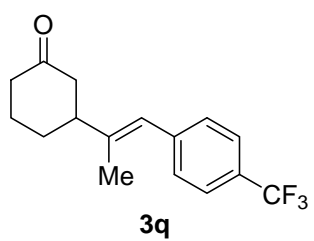

(E)-3-(1-(4-(trifluoromethyl)phenyl)prop-1-en-2-yl)cyclohexan-1-one (3q): Synthesized from 1a and 2q according to the general procedure except for using 1,4-dioxane $(0.8 \mathrm{~mL})$ as the solvent at $90{ }^{\circ} \mathrm{C} .59 \%$ Yield $(33.4$ $\mathrm{mg})$. Colorless oil. $\mathrm{R}_{f}=0.3$ (hexane/EtOAc $\left.=5: 1\right) .{ }^{1} \mathbf{H} \mathbf{N M R}\left(400 \mathrm{MHz}, \mathrm{CDCl}_{3}\right) \delta 7.57(\mathrm{~d}, J=8.1 \mathrm{~Hz}, 2 \mathrm{H}), 7.32(\mathrm{~d}$, $J=8.5 \mathrm{~Hz}, 2 \mathrm{H}), 6.33(\mathrm{~s}, 1 \mathrm{H}), 2.62-2.48(\mathrm{~m}, 2 \mathrm{H}), 2.47-2.40(\mathrm{~m}, 2 \mathrm{H}), 2.38-2.25(\mathrm{~m}, 1 \mathrm{H}), 2.21-2.09(\mathrm{~m}, 1 \mathrm{H})$, $2.04-1.95(\mathrm{~m}, 1 \mathrm{H}), 1.86(\mathrm{~d}, J=1.3 \mathrm{~Hz}, 3 \mathrm{H}), 1.80-1.67(\mathrm{~m}, 2 \mathrm{H}) .{ }^{13} \mathbf{C} \mathbf{N M R}\left(101 \mathrm{MHz}, \mathrm{CDCl}_{3}\right) \delta 211.08,142.70$, 141.62, 129.14, 128.25 (q, $J=32.4 \mathrm{~Hz}), 125.01$ (q, $J=3.8 \mathrm{~Hz}), 124.28$ (q, $J=271.9 \mathrm{~Hz}), 123.79,48.25,46.63$, 41.26, 30.04, 25.22, 15.86. ${ }^{19} \mathbf{F}$ NMR $\left(376 \mathrm{MHz}, \mathrm{CDCl}_{3}\right) \delta-62.40 . \mathbf{I R}\left(\mathrm{KBr}, \mathrm{cm}^{-1}\right) 2941,1713,1614,1325,1122$, 868. HRMS calcd $\mathrm{C}_{16} \mathrm{H}_{18} \mathrm{~F}_{3} \mathrm{O}[\mathrm{M}+\mathrm{H}]^{+}:$: 283.1304. Found: 283.1310 .

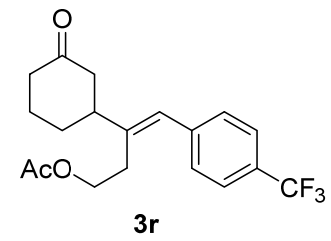

(E)-3-(3-Oxocyclohexyl)-4-(4-(trifluoromethyl)phenyl)but-3-en-1-yl acetate (3r): Synthesized from 1a and 2r according to the general procedure except for using 1,4-dioxane $(0.8 \mathrm{~mL})$ as the solvent at $90{ }^{\circ} \mathrm{C} .61 \%$ Yield $(42.9$ $\mathrm{mg})$. Colorless oil. $\mathrm{R}_{f}=0.6$ (hexane/EtOAc $\left.=1: 1\right) .{ }^{1} \mathbf{H} \mathbf{N M R}\left(400 \mathrm{MHz}, \mathrm{CDCl}_{3}\right) \delta 7.60(\mathrm{~d}, J=8.1 \mathrm{~Hz}, 2 \mathrm{H}), 7.35(\mathrm{~d}$, $J=8.3 \mathrm{~Hz}, 2 \mathrm{H}), 6.47(\mathrm{~s}, 1 \mathrm{H}), 4.17-4.04(\mathrm{~m}, 2 \mathrm{H}), 2.64-2.51(\mathrm{~m}, 4 \mathrm{H}), 2.49-2.28(\mathrm{~m}, 3 \mathrm{H}), 2.20-2.11(\mathrm{~m}, 1 \mathrm{H})$, $2.11-2.03(\mathrm{~m}, 1 \mathrm{H}), 2.01(\mathrm{~s}, 3 \mathrm{H}), 1.80-1.65(\mathrm{~m}, 2 \mathrm{H}) .{ }^{13} \mathbf{C} \mathbf{N M R}\left(101 \mathrm{MHz}, \mathrm{CDCl}_{3}\right) \delta 210.59,170.79,142.65$, 140.99 (q, $J=1.6 \mathrm{~Hz}), 128.78,128.76$ (q, $J=32.5 \mathrm{~Hz}), 126.46,125.27$ (q, $J=3.7 \mathrm{~Hz}), 124.13$ (q, $J=271.9 \mathrm{~Hz})$, 62.29, 47.18, 44.90, 41.17, 30.74, 29.46, 25.15, 20.83. ${ }^{19} \mathbf{F}$ NMR $\left(376 \mathrm{MHz}, \mathrm{CDCl}_{3}\right) \delta$-62.48. IR $\left(\mathrm{KBr}, \mathrm{cm}^{-1}\right) 2956$, 1740, 1712, 1326, 1232, 1122, 867. HRMS calcd $\mathrm{C}_{19} \mathrm{H}_{22} \mathrm{~F}_{3} \mathrm{O}_{3}[\mathrm{M}+\mathrm{H}]^{+}: 355.1516$. Found: 355.1519 .

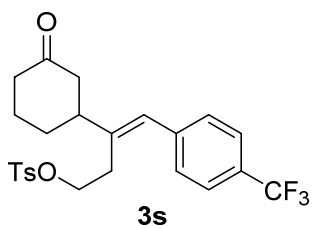

(E)-3-(3-Oxocyclohexyl)-4-(4-(trifluoromethyl)phenyl)but-3-en-1-yl 4-methylbenzenesulfonate (3s): Synthesized from 1a and $\mathbf{2 s}$ according to the general procedure except for using 1,4-dioxane $(0.8 \mathrm{~mL})$ as the solvent at $90{ }^{\circ} \mathrm{C} .62 \%$ Yield $(57.7 \mathrm{mg})$. Colorless oil. $\mathrm{R}_{f}=0.5$ (hexane/EtOAc $\left.=1: 1\right) .{ }^{1} \mathbf{H} \mathbf{N M R}\left(400 \mathrm{MHz}, \mathrm{CDCl}_{3}\right)$ $\delta 7.70(\mathrm{~d}, J=8.3 \mathrm{~Hz}, 2 \mathrm{H}), 7.53(\mathrm{~d}, J=8.1 \mathrm{~Hz}, 2 \mathrm{H}), 7.32(\mathrm{~d}, J=8.1 \mathrm{~Hz}, 2 \mathrm{H}), 7.22(\mathrm{~d}, J=8.0 \mathrm{~Hz}, 2 \mathrm{H}), 6.44(\mathrm{~s}, 1 \mathrm{H})$, $4.19-3.87(\mathrm{~m}, 2 \mathrm{H}), 2.59(\mathrm{t}, J=7.2 \mathrm{~Hz}, 2 \mathrm{H}), 2.49-2.25(\mathrm{~m}, 8 \mathrm{H}), 2.17-2.06(\mathrm{~m}, 1 \mathrm{H}), 2.01-1.91(\mathrm{~m}, 1 \mathrm{H}), 1.76-$ $1.57(\mathrm{~m}, 2 \mathrm{H}) .{ }^{13} \mathbf{C}$ NMR $\left(101 \mathrm{MHz}, \mathrm{CDCl}_{3}\right) \delta 210.22,145.03,141.12,140.66,132.68,129.87,128.84(\mathrm{q}, J=32.5$ 
Hz), 128.70, 127.77, 127.18, 125.26 (q, $J=3.8 \mathrm{~Hz}), 124.07$ (q, $J=272.0 \mathrm{~Hz}), 67.56,46.84,44.59,41.09,30.46$, 29.54, 24.96, 21.55. ${ }^{19}$ F NMR (376 MHz, $\left.\mathrm{CDCl}_{3}\right) \delta$-62.48. IR $\left(\mathrm{KBr}, \mathrm{cm}^{-1}\right)$ 2955, 1711, 1325, 1176, 1122, 962. HRMS calcd $\mathrm{C}_{24} \mathrm{H}_{26} \mathrm{~F}_{3} \mathrm{O}_{4} \mathrm{~S}[\mathrm{M}+\mathrm{H}]^{+}: 467.1498$. Found: 467.1505 .

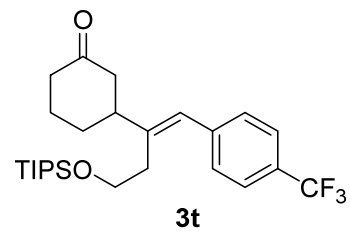

(E)-3-(1-(4-(Trifluoromethyl)phenyl)-4-((triisopropylsilyl)oxy)but-1-en-2-yl)cyclohexan-1-one

(3t):

Synthesized from 1a and $\mathbf{2 t}$ according to the general procedure except for using 1,4-dioxane $(0.8 \mathrm{~mL})$ as the solvent at $90{ }^{\circ} \mathrm{C} .52 \%$ Yield $\left(48.3 \mathrm{mg}\right.$ ). Colorless oil. $\mathrm{R}_{f}=0.5$ (hexane/EtOAc $\left.=3: 1\right) .{ }^{1} \mathbf{H} \mathbf{N M R}\left(400 \mathrm{MHz}, \mathrm{CDCl}_{3}\right) \delta 7.55$ $(\mathrm{d}, J=8.1 \mathrm{~Hz}, 2 \mathrm{H}), 7.40(\mathrm{~d}, J=8.5 \mathrm{~Hz}, 2 \mathrm{H}), 6.42(\mathrm{~s}, 1 \mathrm{H}), 3.80-3.66(\mathrm{~m}, 2 \mathrm{H}), 2.69-2.28(\mathrm{~m}, 7 \mathrm{H}), 2.20-2.01(\mathrm{~m}$, $2 \mathrm{H}), 1.79-1.64(\mathrm{~m}, 2 \mathrm{H}), 1.09-0.92(\mathrm{~m}, 21 \mathrm{H}) .{ }^{13} \mathbf{C}$ NMR $\left(101 \mathrm{MHz}, \mathrm{CDCl}_{3}\right) \delta 211.03,144.55,141.55(\mathrm{q}, J=1.6$ Hz), 128.96, 128.44 (q, $J=32.3 \mathrm{~Hz}), 125.33,125.02$ (q, $J=3.8 \mathrm{~Hz}), 124.24$ (q, $J=271.8 \mathrm{~Hz}), 61.84,47.28,44.90$, 41.27, 33.56, 30.84, 25.24, 17.92, 11.83. ${ }^{19}$ F NMR $\left(376 \mathrm{MHz}, \mathrm{CDCl}_{3}\right) \delta-62.42 . \mathbf{I R}\left(\mathrm{KBr}, \mathrm{cm}^{-1}\right)$ 2944, 2867, 1714, 1325, 1125, 882. HRMS calcd $\mathrm{C}_{26} \mathrm{H}_{40} \mathrm{~F}_{3} \mathrm{O}_{2} \mathrm{Si}[\mathrm{M}+\mathrm{H}]^{+}: 469.2744$. Found: 469.2751.

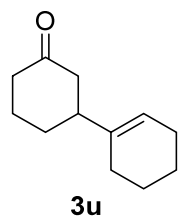

$\left[1,1^{\prime}-\right.$ Bi(cyclohexan)]-1'-en-3-one $(3 \mathbf{u})^{20}$ : Synthesized from $1 \mathbf{a}$ and $\mathbf{2 u}$ according to the general procedure except for using 1,4-dioxane $(0.8 \mathrm{~mL})$ as the solvent at $90{ }^{\circ} \mathrm{C} .43 \%$ Yield $\left(15.4 \mathrm{mg}\right.$ ). Colorless oil. $\mathrm{R}_{f}=0.5$ (hexane/EtOAc $=5: 1) .{ }^{1} \mathbf{H}$ NMR $\left(400 \mathrm{MHz}, \mathrm{CDCl}_{3}\right) \delta 5.44(\mathrm{tdd}, J=3.7,2.4,1.5 \mathrm{~Hz}, 1 \mathrm{H}), 2.43-2.20(\mathrm{~m}, 5 \mathrm{H}), 2.11-1.82(\mathrm{~m}, 6 \mathrm{H})$, $1.72-1.48(\mathrm{~m}, 6 \mathrm{H}) .{ }^{13} \mathrm{C}$ NMR $\left(101 \mathrm{MHz}, \mathrm{CDCl}_{3}\right) \delta 212.16,139.61,120.92,46.75,46.07,41.36,29.98,26.41$, $25.20,25.14,22.93,22.51$.

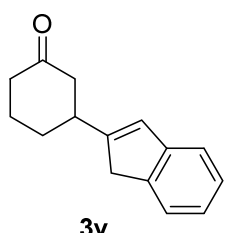

3-(1H-Inden-2-yl)cyclohexan-1-one (3v): Synthesized from 1a and $2 \mathrm{v}$ according to the general procedure except for using 1,4-dioxane $(0.8 \mathrm{~mL})$ as the solvent at $90{ }^{\circ} \mathrm{C} .42 \%$ Yield $(18.0 \mathrm{mg})$. White solid. Melting point: $76-77{ }^{\circ} \mathrm{C}$. $\mathrm{R}_{f}=0.4$ (hexane/EtOAc $\left.=5: 1\right) .{ }^{1} \mathbf{H}$ NMR $\left(400 \mathrm{MHz}, \mathrm{CDCl}_{3}\right) \delta 7.40(\mathrm{dd}, J=7.4,1.0 \mathrm{~Hz}, 1 \mathrm{H}), 7.30(\mathrm{dt}, J=7.4,1.0$ $\mathrm{Hz}, 1 \mathrm{H}), 7.23(\mathrm{dd}, J=7.5,1.1 \mathrm{~Hz}, 1 \mathrm{H}), 7.14(\mathrm{td}, J=7.4,1.3 \mathrm{~Hz}, 1 \mathrm{H}), 6.57$ (dq, $J=2.1,1.3 \mathrm{~Hz}, 1 \mathrm{H}), 3.46-3.27(\mathrm{~m}$, $2 \mathrm{H}), 3.21-2.89(\mathrm{~m}, 1 \mathrm{H}), 2.75-2.60(\mathrm{~m}, 1 \mathrm{H}), 2.57-2.29(\mathrm{~m}, 3 \mathrm{H}), 2.21-1.98(\mathrm{~m}, 2 \mathrm{H}), 1.89-1.70(\mathrm{~m}, 2 \mathrm{H}) .{ }^{13} \mathbf{C}$ NMR $\left(101 \mathrm{MHz}, \mathrm{CDCl}_{3}\right) \delta 210.90,151.90,144.81,142.60,126.44,126.14,124.19,123.54,120.45,47.35,41.32$, 40.29, 39.25, 31.28, 24.91. IR $\left(\mathrm{KBr}, \mathrm{cm}^{-1}\right) 3065,2947,1709,1460,1226,756$. HRMS calcd $\mathrm{C}_{15} \mathrm{H}_{17} \mathrm{O}[\mathrm{M}+\mathrm{H}]^{+}$: 213.1274. Found: 213.1273. 


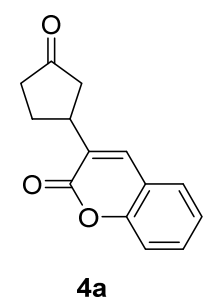

3-(3-Oxocyclopentyl)-2H-chromen-2-one (4a): Synthesized from $\mathbf{1 b}$ and 2a according to the general procedure except for using $15 \mathrm{~mol} \% \mathrm{Pd}(\mathrm{MeCN})_{4}(\mathrm{OTf})_{2}$ and $21 \mathrm{~mol} \% \mathrm{P}(i-\mathrm{Pr})_{3} .51 \%$ Yield $(23.3 \mathrm{mg})$. Colorless oil. $\mathrm{R}_{f}=0.5$ (hexane/EtOAc = 1:1). ${ }^{1} \mathbf{H}$ NMR $\left(400 \mathrm{MHz}, \mathrm{CDCl}_{3}\right) \delta 7.55-7.43(\mathrm{~m}, 3 \mathrm{H}), 7.38-7.23(\mathrm{~m}, 2 \mathrm{H}), 3.52$ (tddd, $J=9.8$, 7.2, 5.8, $1.0 \mathrm{~Hz}, 1 \mathrm{H}), 2.69$ (ddt, $J=19.0,7.4,1.3 \mathrm{~Hz}, 1 \mathrm{H}), 2.55-2.27(\mathrm{~m}, 4 \mathrm{H}), 2.14-1.96(\mathrm{~m}, 1 \mathrm{H}) .{ }^{13} \mathbf{C} \mathbf{~ N M R}$ $\left(101 \mathrm{MHz}, \mathrm{CDCl}_{3}\right) \delta 217.17,160.98,152.99,137.31,131.19,130.31,127.52,124.50,118.98,116.45,43.11,38.20$, 38.00, 27.98. IR $\left(\mathrm{KBr}, \mathrm{cm}^{-1}\right) 2960,1741,1717,1608,1457,1057,757$. HRMS calcd $\mathrm{C}_{14} \mathrm{H}_{13} \mathrm{O}_{3}[\mathrm{M}+\mathrm{H}]^{+}: 229.0859$. Found: 229.0865 .

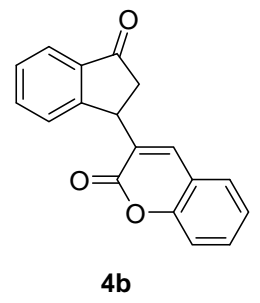

3-(3-Oxo-2,3-dihydro-1H-inden-1-yl)-2H-chromen-2-one (4b): Synthesized from 1c and 2a according to the general procedure except for using $15 \mathrm{~mol} \% \mathrm{Pd}(\mathrm{MeCN})_{4}(\mathrm{OTf})_{2}$ and $21 \mathrm{~mol} \% \mathrm{P}(i-\mathrm{Pr})_{3} .44 \%$ Yield $(24.3 \mathrm{mg})$. Light-yellow oil. $\mathrm{R}_{f}=0.6$ (hexane/EtOAc $\left.=1: 1\right) .{ }^{1} \mathbf{H}$ NMR $\left(400 \mathrm{MHz}, \mathrm{CDCl}_{3}\right) \delta 7.85(\mathrm{~d}, J=7.7 \mathrm{~Hz}, 1 \mathrm{H}), 7.66(\mathrm{td}$, $J=7.5,1.3 \mathrm{~Hz}, 1 \mathrm{H}), 7.54-7.42(\mathrm{~m}, 3 \mathrm{H}), 7.40-7.30(\mathrm{~m}, 3 \mathrm{H}), 7.29-7.21(\mathrm{~m}, 1 \mathrm{H}), 4.81(\mathrm{dd}, J=8.3,3.5 \mathrm{~Hz}, 1 \mathrm{H})$, $3.25(\mathrm{dd}, J=19.1,8.3 \mathrm{~Hz}, 1 \mathrm{H}), 2.68(\mathrm{dd}, J=19.2,3.5 \mathrm{~Hz}, 1 \mathrm{H}) .{ }^{13} \mathbf{C} \mathbf{N M R}\left(101 \mathrm{MHz}, \mathrm{CDCl}_{3}\right) \delta 204.69,161.10$, $154.45,153.13,138.50,137.37,135.09,131.42$, 131.21, 128.49, 127.66, 126.44, 124.53, 124.02, 118.91, 116.53, 43.93, 39.35. IR $\left(\mathrm{KBr}, \mathrm{cm}^{-1}\right) 2925,1712,1607,1457,1176,757$. HRMS calcd $\mathrm{C}_{18} \mathrm{H}_{12} \mathrm{NaO}_{3}[\mathrm{M}+\mathrm{Na}]^{+}: 299.0679$. Found: 299.0678.

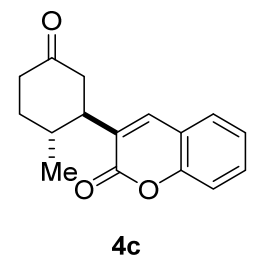

trans-3-(2-Methyl-5-oxocyclohexyl)-2H-chromen-2-one (4c): Synthesized from 1d and 2a according to the general procedure. $62 \%$ Yield $(31.9 \mathrm{mg})$. d.r. $>20: 1$ based on crude NMR of the reaction mixture. The stereochemistry is assigned to be trans as determined by the coupling constant analysis, and the proton signal assignment is based on COSY analysis (vide infra). Colorless oil. $\mathrm{R}_{f}=0.6$ (hexane/EtOAc $=1: 1$ ). ${ }^{1} \mathbf{H}$ NMR (400 $\left.\mathrm{MHz}, \mathrm{CDCl}_{3}\right) \delta 7.57-7.45(\mathrm{~m}, 3 \mathrm{H}), 7.36-7.23(\mathrm{~m}, 2 \mathrm{H}), 2.94$ (t, $\left.J=13.4 \mathrm{~Hz}, 1 \mathrm{H}\right), 2.76$ (ddd, $J=12.7,10.5,4.0$ $\mathrm{Hz}, 1 \mathrm{H}), 2.61-2.42(\mathrm{~m}, 4 \mathrm{H}), 2.13(\mathrm{ddt}, J=12.7,6.3,3.3 \mathrm{~Hz}, 1 \mathrm{H}), 1.53(\mathrm{tdd}, J=13.3,11.4,4.8 \mathrm{~Hz}, 1 \mathrm{H}), 0.94(\mathrm{~d}, J$ $=6.6 \mathrm{~Hz}, 3 \mathrm{H}) .{ }^{13} \mathbf{C}$ NMR $\left(101 \mathrm{MHz}, \mathrm{CDCl}_{3}\right) \delta 210.20,160.46,153.16,139.77,131.23,130.03,127.50,124.43$, 119.05, 116.39, 48.51, 45.10, 41.02, 33.95, 33.48, 19.30. IR (KBr, $\left.\mathrm{cm}^{-1}\right)$ 2957, 1715, 1609, 1281, 1058, 757. HRMS calcd $\mathrm{C}_{16} \mathrm{H}_{17} \mathrm{O}_{3}[\mathrm{M}+\mathrm{H}]^{+}: 257.1172$. Found: 257.1180 . 


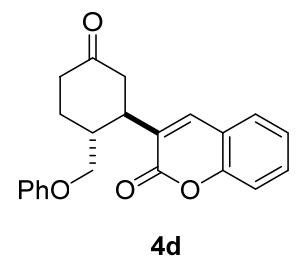

trans-3-(5-Oxo-2-(phenoxymethyl)cyclohexyl)-2H-chromen-2-one (4d): Synthesized from 1e and 2a according to the general procedure. 50\% Yield (34.9 mg). d.r. $>20: 1$ based on crude NMR of the reaction mixture. Colorless oil. $\mathrm{R}_{f}=0.6$ (hexane/EtOAc $\left.=1: 1\right) .{ }^{1} \mathbf{H}$ NMR $\left(400 \mathrm{MHz}, \mathrm{CDCl}_{3}\right) \delta 7.54-7.46(\mathrm{~m}, 2 \mathrm{H}), 7.36(\mathrm{dd}, J=7.8,1.6 \mathrm{~Hz}$, $1 \mathrm{H}), 7.34-7.29(\mathrm{~m}, 1 \mathrm{H}), 7.27-7.15(\mathrm{~m}, 3 \mathrm{H}), 6.88(\mathrm{tt}, J=7.3,1.1 \mathrm{~Hz}, 1 \mathrm{H}), 6.81-6.68(\mathrm{~m}, 2 \mathrm{H}), 3.91(\mathrm{dd}, J=9.5$, $4.2 \mathrm{~Hz}, 1 \mathrm{H}), 3.86(\mathrm{dd}, J=9.5,5.1 \mathrm{~Hz}, 1 \mathrm{H}), 3.23-3.07(\mathrm{~m}, 2 \mathrm{H}), 2.94(\mathrm{dp}, J=15.2,4.6 \mathrm{~Hz}, 1 \mathrm{H}), 2.66-2.45(\mathrm{~m}$, $3 \mathrm{H}), 2.29$ (ddt, $J=13.2,6.0,3.5 \mathrm{~Hz}, 1 \mathrm{H}), 1.96-1.79(\mathrm{~m}, 1 \mathrm{H}) .{ }^{13} \mathbf{C} \mathbf{N M R}\left(101 \mathrm{MHz}, \mathrm{CDCl}_{3}\right) \delta 209.67,160.46$, $158.54,153.24,140.21,131.34,129.39,128.94,127.61,124.46,120.88,118.98,116.35,114.27,69.51,44.70$, 44.49, 40.49, 38.29, 28.65. IR $\left(\mathrm{KBr}, \mathrm{cm}^{-1}\right) 2929,1716,1608,1497,1244,755$. HRMS calcd C ${ }_{22} \mathrm{H}_{21} \mathrm{O}_{4}[\mathrm{M}+\mathrm{H}]^{+}$: 349.1434. Found: 349.1436.

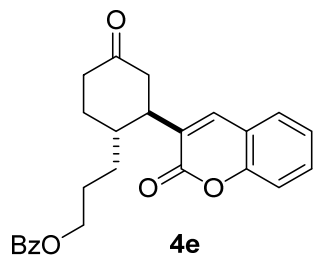

trans-3-(4-Oxo-2-(2-oxo-2H-chromen-3-yl)cyclohexyl)propyl benzoate (4e): Synthesized from 1f and 2a according to the general procedure. 54\% Yield $(43.5 \mathrm{mg})$. d.r. $>20: 1$ based on crude NMR of the reaction mixture. Colorless oil. $\mathrm{R}_{f}=0.5$ (hexane/EtOAc $\left.=1: 1\right) .{ }^{1} \mathbf{H}$ NMR $\left(400 \mathrm{MHz}, \mathrm{CDCl}_{3}\right) \delta 8.00-7.76(\mathrm{~m}, 2 \mathrm{H}), 7.56-7.47(\mathrm{~m}$, $3 \mathrm{H}), 7.43(\mathrm{dd}, J=7.8,1.6 \mathrm{~Hz}, 1 \mathrm{H}), 7.35-7.19(\mathrm{~m}, 4 \mathrm{H}), 4.37-4.19(\mathrm{~m}, 2 \mathrm{H}), 3.04-2.86(\mathrm{~m}, 2 \mathrm{H}), 2.60-2.39(\mathrm{~m}$, 4H), $2.34-2.19(\mathrm{~m}, 1 \mathrm{H}), 1.99-1.83(\mathrm{~m}, 1 \mathrm{H}), 1.78-1.67(\mathrm{~m}, 1 \mathrm{H}), 1.64-1.45(\mathrm{~m}, 2 \mathrm{H}), 1.37-1.21(\mathrm{~m}, 1 \mathrm{H}) .{ }^{13} \mathbf{C}$ NMR $\left(101 \mathrm{MHz}_{\mathrm{CDCl}}\right) \delta$ 209.93, 166.42, 160.38, 153.13, 140.07, 132.79, 131.30, 130.08, 129.71, 129.32, $128.23,127.57,124.48,118.91,116.39,64.53,47.03,45.11,40.57,37.40,30.27,29.36,25.78 . \mathbf{I R}\left(\mathrm{KBr}, \mathrm{cm}^{-1}\right) 2953$, 1716, 1609, 1446, 1276, 713. HRMS calcd $\mathrm{C}_{25} \mathrm{H}_{24} \mathrm{NaO}_{5}[\mathrm{M}+\mathrm{Na}]^{+}:$427.1516. Found: 427.1518.

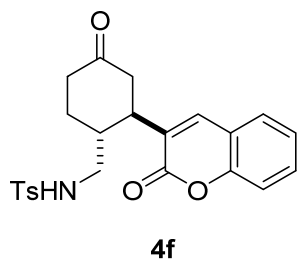

trans-4-Methyl- $N$-((4-oxo-2-(2-oxo-2H-chromen-3-yl)cyclohexyl)methyl)benzenesulfonamide

(4f):

Synthesized from 1g and 2a according to the general procedure. 48\% Yield (44.9 mg). d.r. $>20: 1$ based on crude NMR of the reaction mixture. The overoxidation side product $\mathbf{4 f}$ ' was isolated in $5 \%$ yield as an inseparable mixture with $\mathbf{4 f}\left(\mathbf{4 f}: \mathbf{4 f} \mathbf{f}^{\prime}=1: 0.10\right)$. Colorless oil. $\mathrm{R}_{f}=0.2($ hexane/EtOAc $=1: 1) .{ }^{\mathbf{1}} \mathbf{H} \mathbf{~ N M R}\left(400 \mathrm{MHz}, \mathrm{CDCl}_{3}\right) \delta 7.88$ $(\mathrm{s}, 0.1 \mathrm{H}), 7.65-7.60(\mathrm{~m}, 3.2 \mathrm{H}), 7.58-7.48(\mathrm{~m}, 2.2 \mathrm{H}), 7.37-7.29(\mathrm{~m}, 2.2 \mathrm{H}), 7.22-7.15(\mathrm{~m}, 2.2 \mathrm{H}), 6.41(\mathrm{~d}, J=$ $1.1 \mathrm{~Hz}, 0.1 \mathrm{H}), 5.09-4.95(\mathrm{~m}, 1.1 \mathrm{H}), 3.48-3.40(\mathrm{~m}, 0.1 \mathrm{H}), 3.15-2.93(\mathrm{~m}, 2.2 \mathrm{H}), 2.90-2.66(\mathrm{~m}, 2 \mathrm{H}), 2.54-2.40$ 
(m, 4.2H), 2.35 (s, 3.3H), $2.22-2.11(\mathrm{~m}, 1.2 \mathrm{H}), 1.82-1.66(\mathrm{~m}, 1 \mathrm{H}) .{ }^{13} \mathbf{C}$ NMR $\left(101 \mathrm{MHz}, \mathrm{CDCl}_{3}\right)$ carbon signals of $\mathbf{4 f}$ are found at $\delta 209.00,161.14,153.03,143.50,140.67,136.47,131.63,129.68,128.75,127.84,126.91$, 124.73, 118.94, 116.39, 45.51, 44.78, 42.42, 40.06, 39.13, 28.59, 21.46. IR (KBr, $\left.\mathrm{cm}^{-1}\right) 3272,2925,1714,1608$, 1327, 1160, 732. HRMS calcd $\mathrm{C}_{23} \mathrm{H}_{24} \mathrm{NO}_{5} \mathrm{~S}[\mathrm{M}+\mathrm{H}]^{+}:$426.1370. Found: 426.1377.

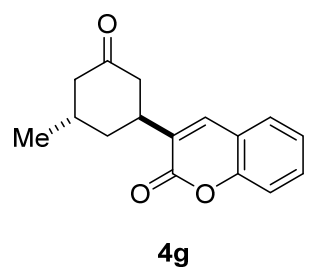

trans-3-(3-Methyl-5-oxocyclohexyl)-2H-chromen-2-one (4g): Synthesized from $1 \mathrm{~h}$ and $2 \mathrm{a}$ according to the general procedure. $40 \%$ Yield $(20.6 \mathrm{mg})$. d.r. $=13: 1$ based on crude NMR of the reaction mixture. The stereochemistry is assigned to be trans as determined by NOESY analysis (vide infra). Colorless oil. $\mathrm{R}_{f}=0.6$ (hexane/EtOAc = 1:1). ${ }^{1} \mathbf{H}$ NMR $\left(400 \mathrm{MHz}, \mathrm{CDCl}_{3}\right) \delta 7.57-7.41(\mathrm{~m}, 3 \mathrm{H}), 7.35-7.19(\mathrm{~m}, 2 \mathrm{H}), 3.61$ (qdd, $J=6.1$, 4.6, $1.1 \mathrm{~Hz}, 0.93 \mathrm{H}), 3.23-3.11(\mathrm{~m}, 0.07 \mathrm{H}), 2.72-2.55(\mathrm{~m}, 2 \mathrm{H}), 2.56-2.43(\mathrm{~m}, 1 \mathrm{H}), 2.29-1.99(\mathrm{~m}, 3 \mathrm{H}), 1.83$ (ddd, $J=13.6,8.5,4.8 \mathrm{~Hz}, 0.93 \mathrm{H}), 1.59(\mathrm{td}, J=12.7,11.3 \mathrm{~Hz}, 0.07 \mathrm{H}), 1.09$ (d, $J=6.1 \mathrm{~Hz}, 0.21 \mathrm{H}), 1.03(\mathrm{~d}, J=6.5$ $\mathrm{Hz}, 2.79 \mathrm{H}) .{ }^{13} \mathbf{C}$ NMR $\left(101 \mathrm{MHz}, \mathrm{CDCl}_{3}\right.$ ) carbon signals of the major diastereomer are found at $\delta 211.22,160.76$, $152.92,138.53,131.45,131.10,127.68,124.43,118.99,116.33,49.09,44.55,35.62,35.48,29.22,20.71$. IR (KBr, $\left.\mathrm{cm}^{-1}\right) 2956,1714,1609,1457,1176,757$. HRMS calcd $\mathrm{C}_{16} \mathrm{H}_{16} \mathrm{NaO}_{3}[\mathrm{M}+\mathrm{Na}]^{+}: 279.0992$. Found: 279.0992.

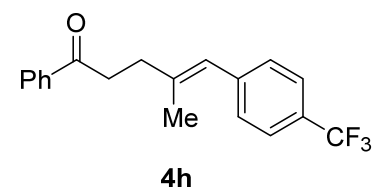

(E)-4-Methyl-1-phenyl-5-(4-(trifluoromethyl)phenyl)pent-4-en-1-one (4h): Synthesized from $1 \mathrm{i}$ and $2 \mathrm{q}$ according to the general procedure except for using 5 equiv. of $\mathbf{1 i}, 20 \mathrm{~mol} \% \mathrm{P}(i-\mathrm{Pr})_{3}$ and 1,4-dioxane $(0.8 \mathrm{~mL})$ as the solvent at $90{ }^{\circ} \mathrm{C}$. The product $\mathbf{4 h}$, the overoxidation product $\mathbf{4} \mathbf{h}$ ' and the di-alkenylation product were isolated in $7.6 \%(4.8 \mathrm{mg}), 42 \%(26.5 \mathrm{mg})$ and $3.1 \%$ yields $(3.1 \mathrm{mg})$, respectively. The overoxidation product $\mathbf{4 h}$ ' can be quantitatively transferred to $\mathbf{4 h}$ (vide infra). $53 \%$ Total yield (mono:di $=16: 1$ ). Colorless oil. $\mathrm{R}_{f}=0.4$ (hexane/EtOAc = 10:1). ${ }^{1} \mathbf{H}$ NMR $\left(400 \mathrm{MHz}, \mathrm{CDCl}_{3}\right) \delta 8.06-7.90(\mathrm{~m}, 2 \mathrm{H}), 7.63-7.53(\mathrm{~m}, 3 \mathrm{H}), 7.48(\mathrm{dd}, J=8.4$, $6.9 \mathrm{~Hz}, 2 \mathrm{H}), 7.31(\mathrm{~d}, J=8.1 \mathrm{~Hz}, 2 \mathrm{H}), 6.34(\mathrm{~s}, 1 \mathrm{H}), 3.38-3.05(\mathrm{~m}, 2 \mathrm{H}), 2.64(\mathrm{td}, J=7.6,1.2 \mathrm{~Hz}, 2 \mathrm{H}), 1.92(\mathrm{~d}, J=$ $1.3 \mathrm{~Hz}, 3 \mathrm{H}) .{ }^{13} \mathbf{C}$ NMR $\left(101 \mathrm{MHz}, \mathrm{CDCl}_{3}\right) \delta 199.41,141.80,140.06,136.86,133.11,128.97,128.64,128.03$, $127.96(\mathrm{q}, J=32.5 \mathrm{~Hz}), 124.94(\mathrm{q}, J=3.8 \mathrm{~Hz}), 124.38,124.29(\mathrm{q}, J=271.8 \mathrm{~Hz}), 37.04,34.78,18.03 .{ }^{19} \mathbf{F}$ NMR $\left(376 \mathrm{MHz}, \mathrm{CDCl}_{3}\right) \delta-62.33$. IR $\left(\mathrm{KBr}, \mathrm{cm}^{-1}\right) 3061,2914,1686,1326,1121,1068$, 867. HRMS calcd $\mathrm{C}_{19} \mathrm{H}_{18} \mathrm{~F}_{3} \mathrm{O}$ $[\mathrm{M}+\mathrm{H}]^{+}:$319.1304. Found: 319.1303 .

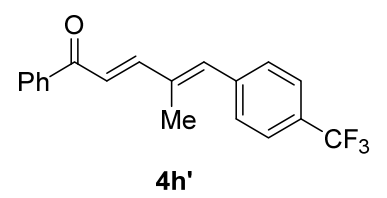

(2E,4E)-4-Methyl-1-phenyl-5-(4-(trifluoromethyl)phenyl)penta-2,4-dien-1-one (4h'): Isolated as the side product from the synthesis of $4 \mathbf{h}$ in $42 \%$ yield $(26.5 \mathrm{mg})$. Light-yellow solid. Melting point: $153-155{ }^{\circ} \mathrm{C} . \mathrm{R}_{f}=0.3$ (hexane/EtOAc = 10:1). ${ }^{1} \mathbf{H}$ NMR $\left(400 \mathrm{MHz}, \mathrm{CDCl}_{3}\right) \delta 8.10-7.91(\mathrm{~m}, 2 \mathrm{H}), 7.70-7.55(\mathrm{~m}, 4 \mathrm{H}), 7.54-7.44$ (m, 
4H), $7.11(\mathrm{~d}, J=15.4 \mathrm{~Hz}, 1 \mathrm{H}), 6.97(\mathrm{~s}, 1 \mathrm{H}), 2.16(\mathrm{~d}, J=1.2 \mathrm{~Hz}, 3 \mathrm{H}) .{ }^{13} \mathbf{C}$ NMR $\left(101 \mathrm{MHz}, \mathrm{CDCl}_{3}\right) \delta 190.57$, $149.14,140.20,138.28,138.19,136.52,132.74,129.60,129.47$ (q, $J=32.5 \mathrm{~Hz}), 128.59,128.42,125.24$ (q, $J=3.8$ $\mathrm{Hz}), 124.02(\mathrm{q}, J=272.0 \mathrm{~Hz}), 122.52,13.93 .{ }^{19} \mathbf{F}$ NMR $\left(376 \mathrm{MHz}, \mathrm{CDCl}_{3}\right) \delta-62.58 . \mathbf{I R}\left(\mathrm{KBr}, \mathrm{cm}^{-1}\right) 3062,1655$, 1587, 1330, 1111, 861. HRMS calcd $\mathrm{C}_{19} \mathrm{H}_{16} \mathrm{~F}_{3} \mathrm{O}[\mathrm{M}+\mathrm{H}]^{+}: 317.1148$. Found: 317.1153 .

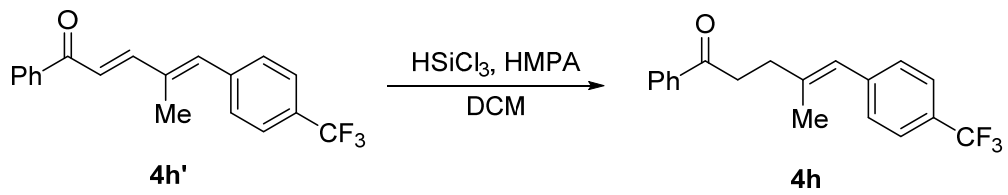

The selective 1,4-reduction was performed based on a literature procedure. ${ }^{21} \mathrm{~A} 4-\mathrm{mL}$ vial was flame-dried and charged with 4h' (1 equiv., $0.084 \mathrm{mmol}, 26.5 \mathrm{mg})$, DCM $(0.4 \mathrm{~mL})$ and HMPA (20 mol \%, $0.017 \mathrm{mmol}, 3.0 \mu \mathrm{L})$. $\mathrm{HSiCl}_{3}$ (2 equiv., $0.168 \mathrm{mmol}, 17.0 \mu \mathrm{L}$ ) was dissolved in $\mathrm{DCM}(0.1 \mathrm{~mL})$, and the solution was added to the reaction vial dropwise at $0{ }^{\circ} \mathrm{C}$. The mixture was stirred at $0{ }^{\circ} \mathrm{C}$ until the yellow color faded $(90 \mathrm{~min})$. The mixture was quenched with $\mathrm{NaHCO}_{3}$ and extracted with $\mathrm{Et}_{2} \mathrm{O}$. The combined organic layers were filtered through Celite, and the filtrate was concentrated. The residue was purified by column chromatography to give product $\mathbf{4 h}$ as a colorless oil in a quantitative yield $(26.7 \mathrm{mg})$.

\section{Derivatization of the alkenylation product}

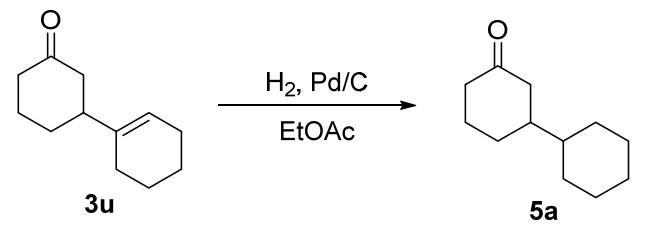

[1,1'-Bi(cyclohexan)]-3-one (5a) ${ }^{2}$ : An 8-mL culture tube was charged with $\mathrm{Pd} / \mathrm{C}(10 \mathrm{wt} . \%, 5 \mathrm{~mol} \%, 0.005 \mathrm{mmol}$, $5.3 \mathrm{mg}$ ) and replaced with $\mathrm{N}_{2} .3 \mathrm{u}$ (1 equiv., $0.1 \mathrm{mmol}, 17.8 \mathrm{mg}$ ) was dissolved in EtOAc $(1.5 \mathrm{~mL})$, and the solution was added via syringe. The atmosphere was replaced with $\mathrm{H}_{2}$ using a balloon, and the mixture was stirred under $\mathrm{H}_{2}$ overnight. The mixture was filtered through Celite, eluting with EtOAc. The filtrate was concentrated, and the residue was purified by column chromatography to give the product as a colorless oil in $97 \%$ yield $(17.5 \mathrm{mg}) . \mathrm{R}_{f}=$ 0.5 (hexane/EtOAc = 5:1). ${ }^{1} \mathbf{H}$ NMR $\left(400 \mathrm{MHz}, \mathrm{CDCl}_{3}\right) \delta 2.51-2.32(\mathrm{~m}, 2 \mathrm{H}), 2.24$ (dddd, $J=14.1,12.9,6.2,1.3$ $\mathrm{Hz}, 1 \mathrm{H}), 2.15-2.02(\mathrm{~m}, 2 \mathrm{H}), 1.93-1.82(\mathrm{~m}, 1 \mathrm{H}), 1.79-1.50(\mathrm{~m}, 7 \mathrm{H}), 1.38$ (tdd, $J=12.8,11.5,3.6 \mathrm{~Hz}, 1 \mathrm{H}), 1.29$ - $1.06(\mathrm{~m}, 4 \mathrm{H}), 1.05-0.87(\mathrm{~m}, 2 \mathrm{H}) .{ }^{13} \mathbf{C}$ NMR $\left(101 \mathrm{MHz}, \mathrm{CDCl}_{3}\right) \delta$ 212.87, 45.54, 44.64, 42.63, 41.59, 29.92, $29.82,28.39,26.55,26.53,26.49,25.60$.

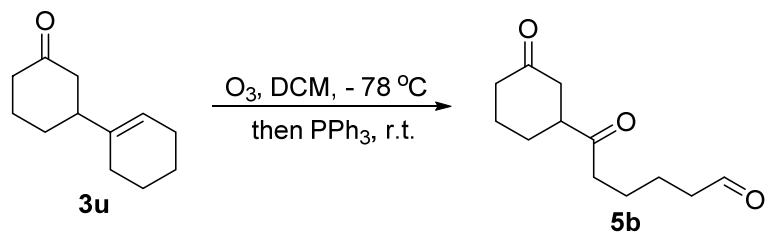


6-Oxo-6-(3-oxocyclohexyl)hexanal (5b): An 8-mL culture tube was charged with $\mathbf{3 u}$ (1 equiv., $0.1 \mathrm{mmol}, 17.8 \mathrm{mg}$ ) and DCM $(2 \mathrm{~mL})$, and the solution was cooled to $-78{ }^{\circ} \mathrm{C} . \mathrm{PPh}_{3}(10$ equiv., $1 \mathrm{mmol}, 262 \mathrm{mg}$ ) was dissolved in minimum amount of DCM. The 3u solution was treated with an $\mathrm{O}_{3}$ flow until the solution stayed blue. The $\mathrm{O}_{3}$ flow was then stopped, and the $\mathrm{PPh}_{3}$ solution was added to the tube immediately. The mixture was warmed to room temperature and stirred overnight. The mixture was concentrated, and the residue was purified by column chromatography to give the product as a colorless oil in $59 \%$ yield $(12.4 \mathrm{mg}) . \mathrm{R}_{f}=0.4$ (hexane/EtOAc $\left.=1: 1\right) .{ }^{1} \mathbf{H}$ NMR (400 MHz, $\left.\mathrm{CDCl}_{3}\right) \delta 9.77(\mathrm{t}, J=1.5 \mathrm{~Hz}, 1 \mathrm{H}), 2.96-2.79(\mathrm{~m}, 1 \mathrm{H}), 2.67-2.21(\mathrm{~m}, 8 \mathrm{H}), 2.15-1.98(\mathrm{~m}, 2 \mathrm{H})$, 1.82 - 1.55 (m, 6H). ${ }^{13} \mathbf{C}$ NMR $\left(101 \mathrm{MHz}, \mathrm{CDCl}_{3}\right) \delta 210.10,209.96,202.02,50.21,43.66,42.53,40.91,40.71$, 27.38, 24.92, 22.93, 21.47. IR $\left(\mathrm{KBr}, \mathrm{cm}^{-1}\right)$ 2941, 1712, 1450, 1226, 1118, 976. HRMS calcd $\mathrm{C}_{12} \mathrm{H}_{19} \mathrm{O}_{3}[\mathrm{M}+\mathrm{H}]^{+}$: 211.1329. Found: 211.1332.

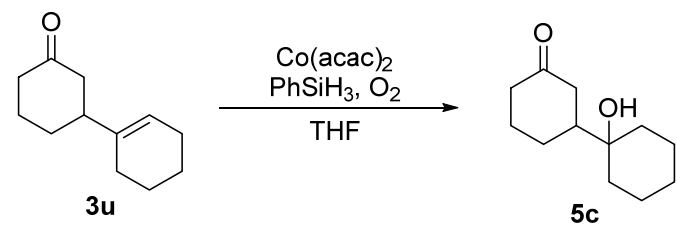

1'-Hydroxy-[1,1'-bi(cyclohexan)]-3-one (5c): The reaction was performed based on a literature procedure. ${ }^{22} \mathrm{An}$ 8 - $\mathrm{mL}$ culture tube was charged with $\mathrm{Co}(\mathrm{acac})_{2}(20 \mathrm{~mol} \%, 0.02 \mathrm{mmol}, 5.1 \mathrm{mg})$, THF $(0.5 \mathrm{~mL}), 3 \mathbf{u}$ (1 equiv., 0.1 mmol, $17.8 \mathrm{mg}$ ) and $\mathrm{PhSiH}_{3}$ (2 equiv., $0.2 \mathrm{mmol}, 24.6 \mu \mathrm{L}$ ). The atmosphere was replaced with $\mathrm{O}_{2}$ using a balloon, and the mixture was stirred under $\mathrm{O}_{2}$ overnight. The mixture was concentrated, and the residue was purified by column chromatography to give the product as a colorless oil in $80 \%$ yield $\left(15.7 \mathrm{mg}\right.$ ). $\mathrm{R}_{f}=0.5$ (hexane/EtOAc $=$ 1:1). ${ }^{1} \mathrm{H}$ NMR $\left(400 \mathrm{MHz}, \mathrm{CDCl}_{3}\right) \delta 2.49(\mathrm{ddt}, J=13.8,4.2,2.1 \mathrm{~Hz}, 1 \mathrm{H}), 2.37$ (dddd, $\left.J=14.2,6.4,2.6,1.6 \mathrm{~Hz}, 1 \mathrm{H}\right)$, $2.32-2.20(\mathrm{~m}, 2 \mathrm{H}), 2.13$ (ddq, $J=11.9,5.6,3.0 \mathrm{~Hz}, 1 \mathrm{H}), 1.99$ (dddd, $J=12.7,5.3,3.6,1.8 \mathrm{~Hz}, 1 \mathrm{H}), 1.75$ (ddt, $J=$ $12.8,11.5,3.7 \mathrm{~Hz}, 1 \mathrm{H}), 1.68-1.51(\mathrm{~m}, 8 \mathrm{H}), 1.50-1.31(\mathrm{~m}, 3 \mathrm{H}), 1.29-1.14(\mathrm{~m}, 2 \mathrm{H}) .{ }^{13} \mathbf{C}$ NMR $(101 \mathrm{MHz}$, $\left.\mathrm{CDCl}_{3}\right) \delta 212.90,72.33,48.32,42.17,41.33,34.95,34.61,25.65,25.24,24.72,21.87,21.78 . \mathrm{IR}\left(\mathrm{KBr}, \mathrm{cm}^{-1}\right) 3463$, 2932, 1702, 1449, 1150, 960. HRMS calcd $\mathrm{C}_{12} \mathrm{H}_{21} \mathrm{O}_{2}[\mathrm{M}+\mathrm{H}]^{+}:$197.1536. Found: 197.1533.

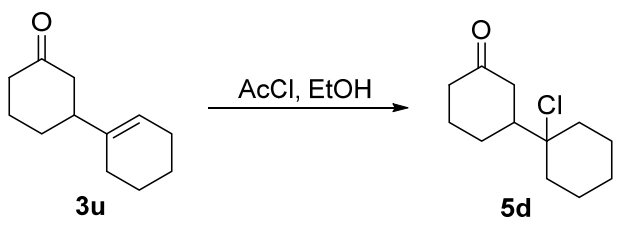

1'-Chloro-[1,1'-bi(cyclohexan)]-3-one (5d): The reaction was performed based on a literature procedure. ${ }^{23} \mathrm{~A}$ 2-mL vial was flame-dried and charged with $3 \mathbf{u}$ (1 equiv., $0.1 \mathrm{mmol}, 17.8 \mathrm{mg}$ ) and $\mathrm{EtOH}$ (8 equiv., $0.8 \mathrm{mmol}, 46.7$ $\mu \mathrm{L}$ ). The mixture was cooled to $0{ }^{\circ} \mathrm{C}$, and $\mathrm{AcCl}(8$ equiv., $0.8 \mathrm{mmol}, 56.9 \mu \mathrm{L}$ ) was added dropwise. The mixture was heated at $30{ }^{\circ} \mathrm{C}$ for $1 \mathrm{~h}$. The mixture was concentrated, and the residue was purified by column chromatography to give the product as an colorless oil in $94 \%$ yield $\left(20.2 \mathrm{mg}\right.$ ). $\mathrm{R}_{f}=0.4$ (hexane/EtOAc $\left.=5: 1\right) .{ }^{1} \mathbf{H}$ NMR (400 MHz, $\left.\mathrm{CDCl}_{3}\right) \delta 2.59$ (ddt, $\left.J=14.1,4.2,2.1 \mathrm{~Hz}, 1 \mathrm{H}\right), 2.50-2.35$ (m, 2H), 2.29 (tdd, $J=14.3,6.2,1.1$ $\mathrm{Hz}, 1 \mathrm{H}), 2.20-2.05(\mathrm{~m}, 3 \mathrm{H}), 2.01-1.87$ (m, 2H), $1.82-1.39$ (m, 9H), $1.24-1.09$ (m, 1H). ${ }^{13} \mathbf{C}$ NMR (101 MHz, $\left.\mathrm{CDCl}_{3}\right) \delta 211.76,77.86,49.99,42.69,41.19,37.88,37.45,25.23,24.53,22.19,21.99$. IR $\left(\mathrm{KBr}, \mathrm{cm}^{-1}\right) 2932,2863$, $1713,1448,1261,890$. HRMS calcd $\mathrm{C}_{12} \mathrm{H}_{20} \mathrm{ClO}[\mathrm{M}+\mathrm{H}]^{+}: 215.1197$. Found: 215.1204 . 


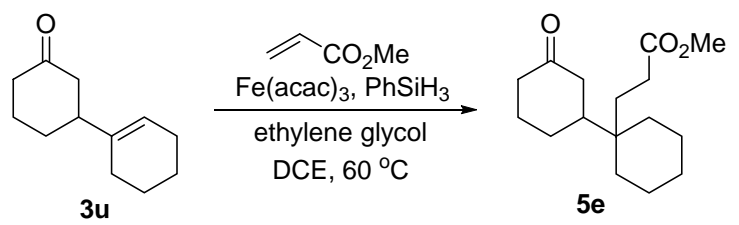

Methyl 3-(3'-oxo-[1,1'-bi(cyclohexan)]-1-yl)propanoate (5e): The reaction was performed based on a literature procedure. ${ }^{24}$ An $8-\mathrm{mL}$ culture tube was charged with $\mathrm{Fe}(\text { acac) })_{3}\left(1\right.$ equiv., $0.1 \mathrm{mmol}, 35.3 \mathrm{mg}$ ) and refilled with $\mathrm{N}_{2}$. DCE $(0.8 \mathrm{~mL})$, ethylene glycol $(0.16 \mathrm{~mL}), 3 \mathbf{u}(1$ equiv., $0.1 \mathrm{mmol}, 17.8 \mathrm{mg})$, methyl acrylate (3 equiv., $0.3 \mathrm{mmol}$, $27.2 \mu \mathrm{L}$ ) and $\mathrm{PhSiH}_{3}\left(1.5\right.$ equiv., $0.15 \mathrm{mmol}, 18.5 \mu \mathrm{L}$ ) were added via syringe. The mixture was heated at $60{ }^{\circ} \mathrm{C}$ for $1 \mathrm{~h}$. After cooling to room temperature, the mixture was diluted with water and brine, and extracted with $\mathrm{Et}_{2} \mathrm{O}$. The combined organic layers were dried and concentrated, and the residue was purified by column chromatography to give the product as a colorless oil in $73 \%$ yield $(19.4 \mathrm{mg}) . \mathrm{R}_{f}=0.5$ (hexane/EtOAc $\left.=3: 1\right) .{ }^{1} \mathbf{H} \mathbf{~ N M R}(400 \mathrm{MHz}$, $\left.\mathrm{CDCl}_{3}\right) \delta 3.68(\mathrm{~s}, 3 \mathrm{H}), 2.43-2.31(\mathrm{~m}, 2 \mathrm{H}), 2.30-2.17(\mathrm{~m}, 3 \mathrm{H}), 2.16-2.06(\mathrm{~m}, 2 \mathrm{H}), 1.93-1.82(\mathrm{~m}, 1 \mathrm{H}), 1.82-$ $1.63(\mathrm{~m}, 3 \mathrm{H}), 1.61-1.18(\mathrm{~m}, 12 \mathrm{H}) .{ }^{13} \mathbf{C}$ NMR $\left(101 \mathrm{MHz}, \mathrm{CDCl}_{3}\right) \delta 212.77,174.60,51.64,43.57,42.42,41.44$, $36.68,31.31,31.29,28.26,27.73,26.02,25.75,25.06,21.13,21.07 . \mathbf{I R}\left(\mathrm{KBr}, \mathrm{cm}^{-1}\right) 2932,1738,1712,1435,1173$, 760. HRMS calcd $\mathrm{C}_{16} \mathrm{H}_{27} \mathrm{O}_{3}[\mathrm{M}+\mathrm{H}]^{+}: 267.1955$. Found: 267.1962 .

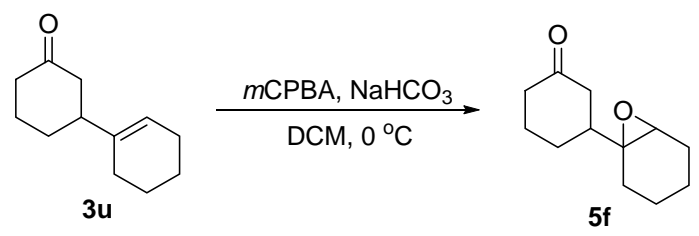

3-(7-Oxabicyclo[4.1.0]heptan-1-yl)cyclohexan-1-one (5f): A 4-mL vial was charged with 3u (1 equiv., $0.1 \mathrm{mmol}$, $17.8 \mathrm{mg}), \mathrm{DCM}(1 \mathrm{~mL})$ and $\mathrm{NaHCO}_{3}(1.5$ equiv., $0.15 \mathrm{mmol}, 12.6 \mathrm{mg})$. The mixture was cooled to $0{ }^{\circ} \mathrm{C}$ before mCPBA (1.1 equiv., $0.11 \mathrm{mmol}, 24.6 \mathrm{mg}$ ) was added. After being stirred at $0{ }^{\circ} \mathrm{C}$ for $2 \mathrm{~h}$, the mixture was then diluted with DCM and quenched with $\mathrm{Na}_{2} \mathrm{SO}_{3}$. The mixture was further stirred for 10 min and then extracted with DCM. The combined organic layers were washed with brine, dried and concentrated. The residue was purified by column chromatography to give the product as a colorless oil in $96 \%$ yield $(18.6 \mathrm{mg})$. d.r. was not identifiable in ${ }^{1} \mathrm{H}$ NMR; it was estimated to be $1: 1$ based on ${ }^{13} \mathrm{C}$ NMR of the isolated product. $\mathrm{R}_{f}=0.4$ (hexane/EtOAc $=3: 1$ ). ${ }^{1} \mathbf{H}$ NMR $\left(400 \mathrm{MHz}, \mathrm{CDCl}_{3}\right) \delta 3.00(\mathrm{t}, J=3.1 \mathrm{~Hz}, 1 \mathrm{H}), 2.49-2.33(\mathrm{~m}, 2 \mathrm{H}), 2.30-2.05(\mathrm{~m}, 3 \mathrm{H}), 2.01-1.89(\mathrm{~m}, 2 \mathrm{H})$, $1.88-1.36(\mathrm{~m}, 8 \mathrm{H}), 1.30-1.15(\mathrm{~m}, 2 \mathrm{H}) .{ }^{13} \mathbf{C}$ NMR $\left(101 \mathrm{MHz}, \mathrm{CDCl}_{3}\right)$ carbon signals are found at $\delta 210.93$, $210.91,61.55,61.39,57.82,57.12,46.10,45.38,43.71,42.72$, 41.34, 41.22, 26.68, 25.98, 25.60, 25.09, 24.98, 24.97, 24.85, 24.84, 20.32, 20.26, 19.48. IR $\left(\mathrm{KBr}, \mathrm{cm}^{-1}\right)$ 2937, 1712, 1448, 1317, 1218, 968. HRMS calcd $\mathrm{C}_{12} \mathrm{H}_{19} \mathrm{O}_{2}[\mathrm{M}+\mathrm{H}]^{+}:$195.1380. Found: 195.1376. 


\section{References}

(1) Thomas, A. A.; Wang, H.; Zahrt, A. F.; Denmark, S. E. J. Am. Chem. Soc. 2017, 139, 14389.

(2) Wang, C.; Dong, G. J. Am. Chem. Soc. 2018, 140, 6057.

(3) Audisio, D.; Messaoudi, S.; Brion, J.-D.; Alami, M. Eur. J. Org. Chem. 2010, 1046.

(4) Chaudhuri, S. K.; Roy, S.; Bhar, S. Beilstein J. Org. Chem. 2012, 8, 323.

(5) Chen, L.; Hu, T.-S.; Yao, Z.-J. Eur. J. Org. Chem. 2008, 6175.

(6) Timári, G.; Soós, T.; Hajós, G. Synlett 1997, 1067.

(7) Wu, G.; Wangelin, A. J. Chem. Sci. 2018, 9, 1795.

(8) Telvekar, V. N.; Takale, B. S. Tetrahedron Lett. 2011, 52, 2394.

(9) Zhan, F.; Liang, G. Angew. Chem., Int. Ed. 2013, 52, 1266.

(10) Marshall, J. A.; Partridge, J. J. Tetrahedron 1969, 25, 2159.

(11) Harayama, T.; Nakatsuka, K.; Hishioka, H.; Murakami, K.; Hayashida, N.; Ishii, H. Chem. Pharm. Bull. 1994, 42, 2170.

(12) Sharma, U.; Naveen, T.; Maji, A.; Manna, S.; Maiti, D. Angew. Chem., Int. Ed. 2013, 52, 12669.

(13) Bian, T.; Vijendra, K. C.; Wang, Y.; Meacham, A.; Hati, S.; Cogle, C. R.; Sun, H.; Xing, C. J. Med. Chem. 2018, 61, 6892 .

(14) Yuan, W.; Ma, S. Org. Biomol. Chem. 2012, 10, 7266.

(15) Kim, H. R.; Jung, I. G.; Yoo, K.; Jang, K.; Lee, E. S.; Yun, J.; Son, S. U. Chem. Commun. 2010, 758.

(16) Thorpe, S. B.; Guo, X.; Santos, W. L. Chem. Commun. 2011, 424.

(17) Marvell, E. N.; Stummer, D.; Rowell, C. Tetrahedron 1966, 22, 861.

(18) Sumitomo Chemical Co.; Mitsudera, H. Patent WO2009/5110 A2, 2009, 143.

(19) Herath, A.; Thompson, B. B.; Montgomery, J. J. Am. Chem. Soc. 2007, 129, 8712.

(20) Kende, A. S.; Jungheim, L. N. Tetrahedron Lett. 1980, 21, 3849.

(21) Sugiura, M.; Sato, N.; Kotani, S.; Nakajima, M. Chem. Commun. 2008, 4309.

(22) Isayama, S.; Mukaiyama, T. Chem. Lett. 1989, 18, 1071.

(23) Yadav, V. K.; Babu, K. G. Eur. J. Org. Chem. 2005, 452.

(24) Lo, J. C.; Yabe, Y.; Baran, P. S. J. Am. Chem. Soc. 2014, 136, 1304. 


\section{8. ${ }^{1} \mathrm{H}$ and ${ }^{13} \mathrm{C}$ NMR spectra}

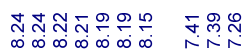

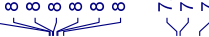
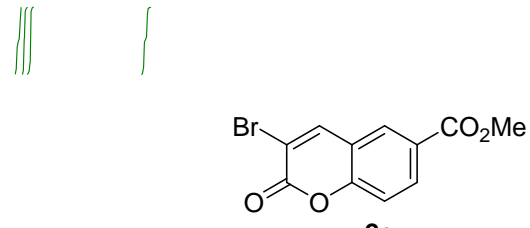

$2 e$

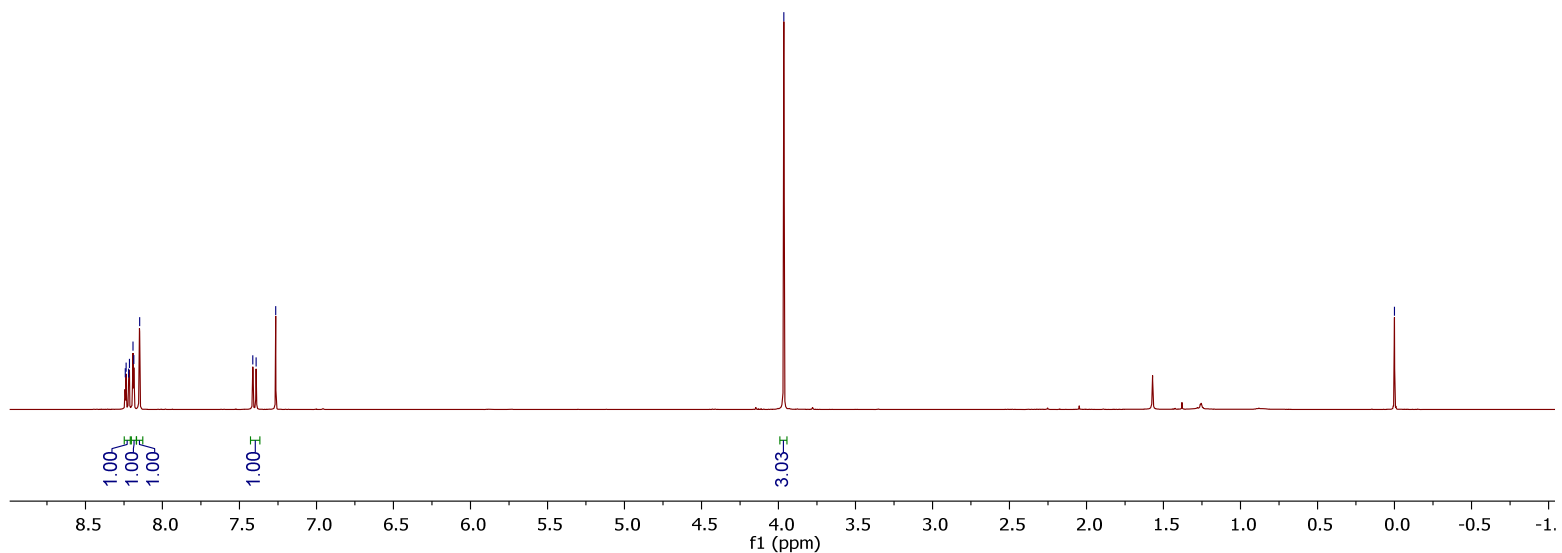

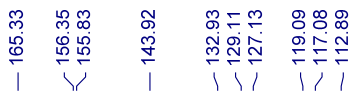

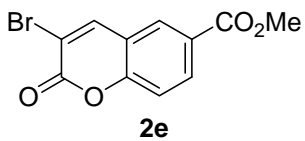

$2 \mathrm{e}$

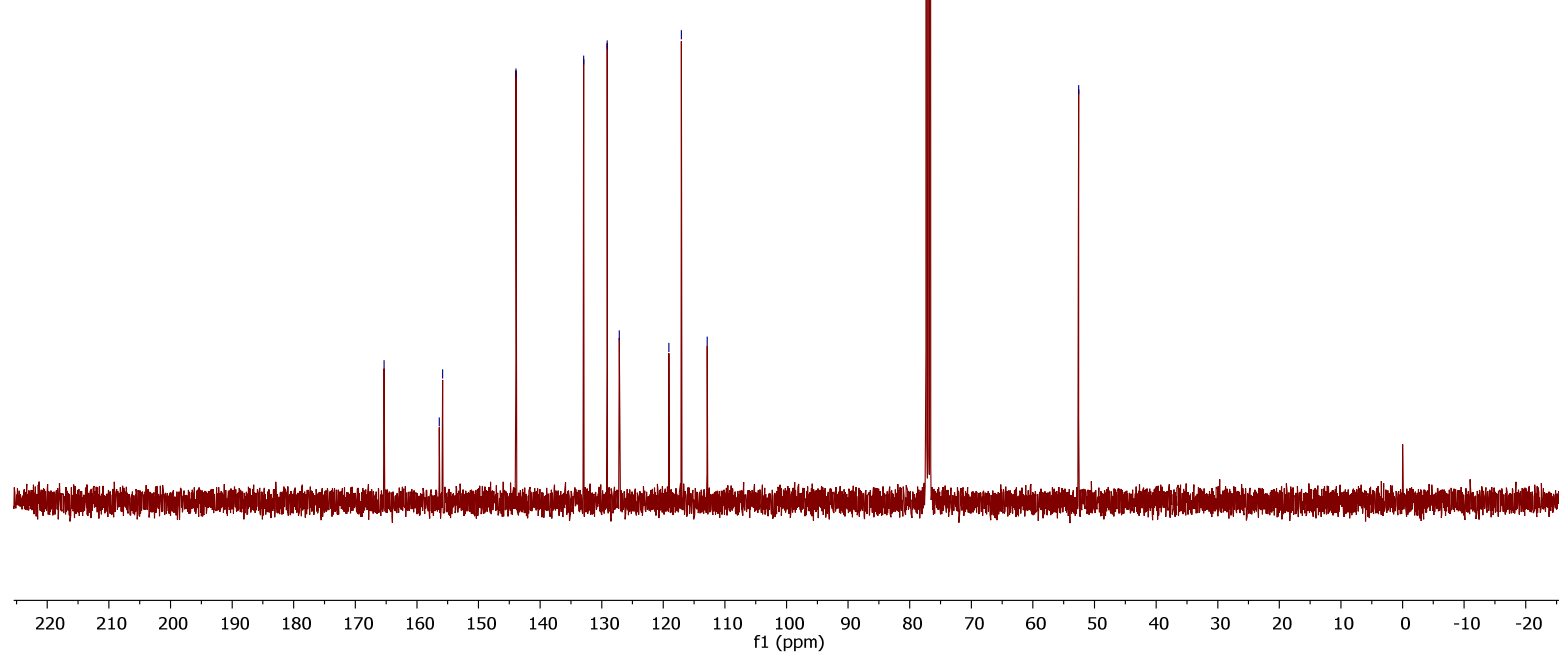




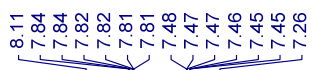
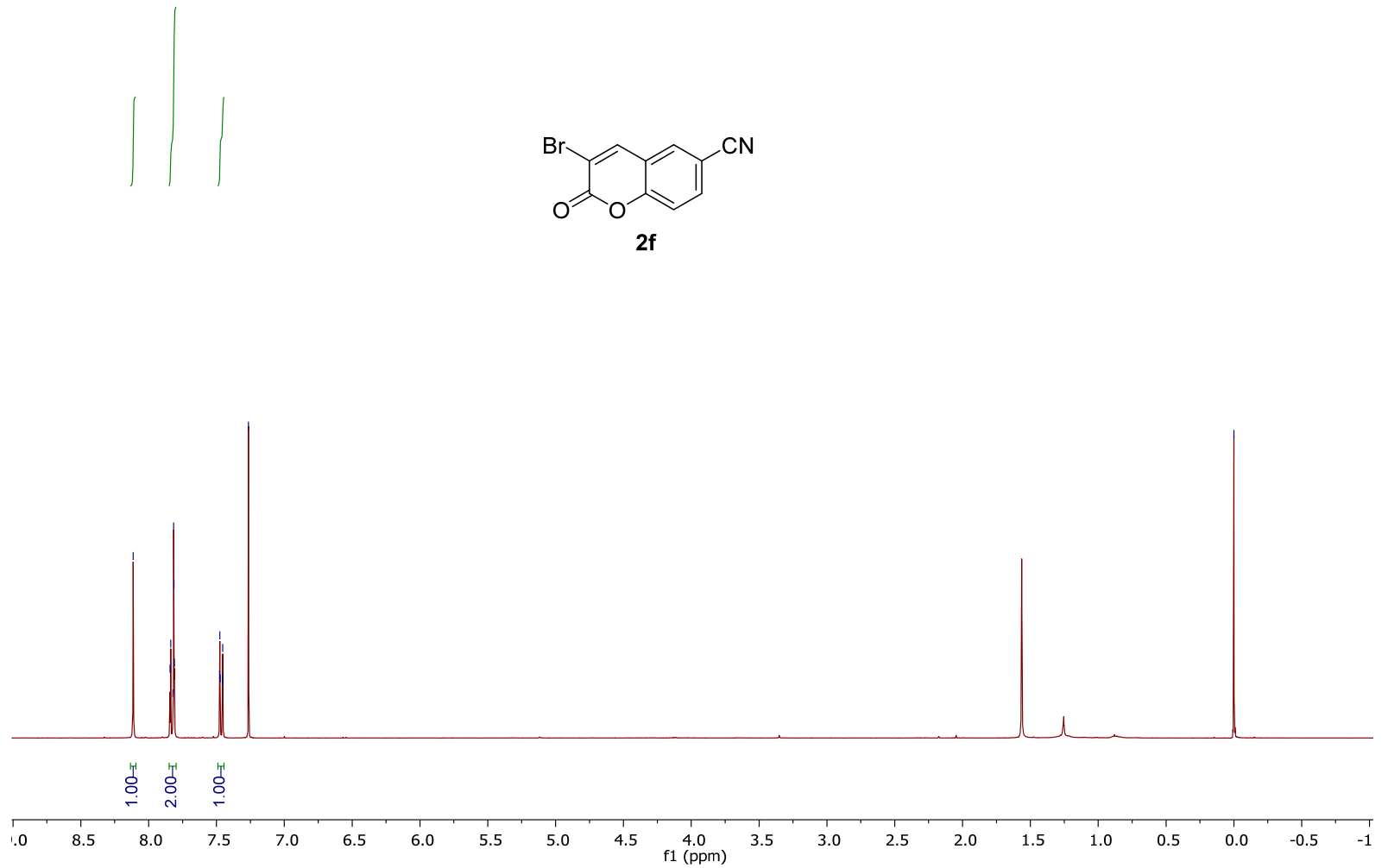

V
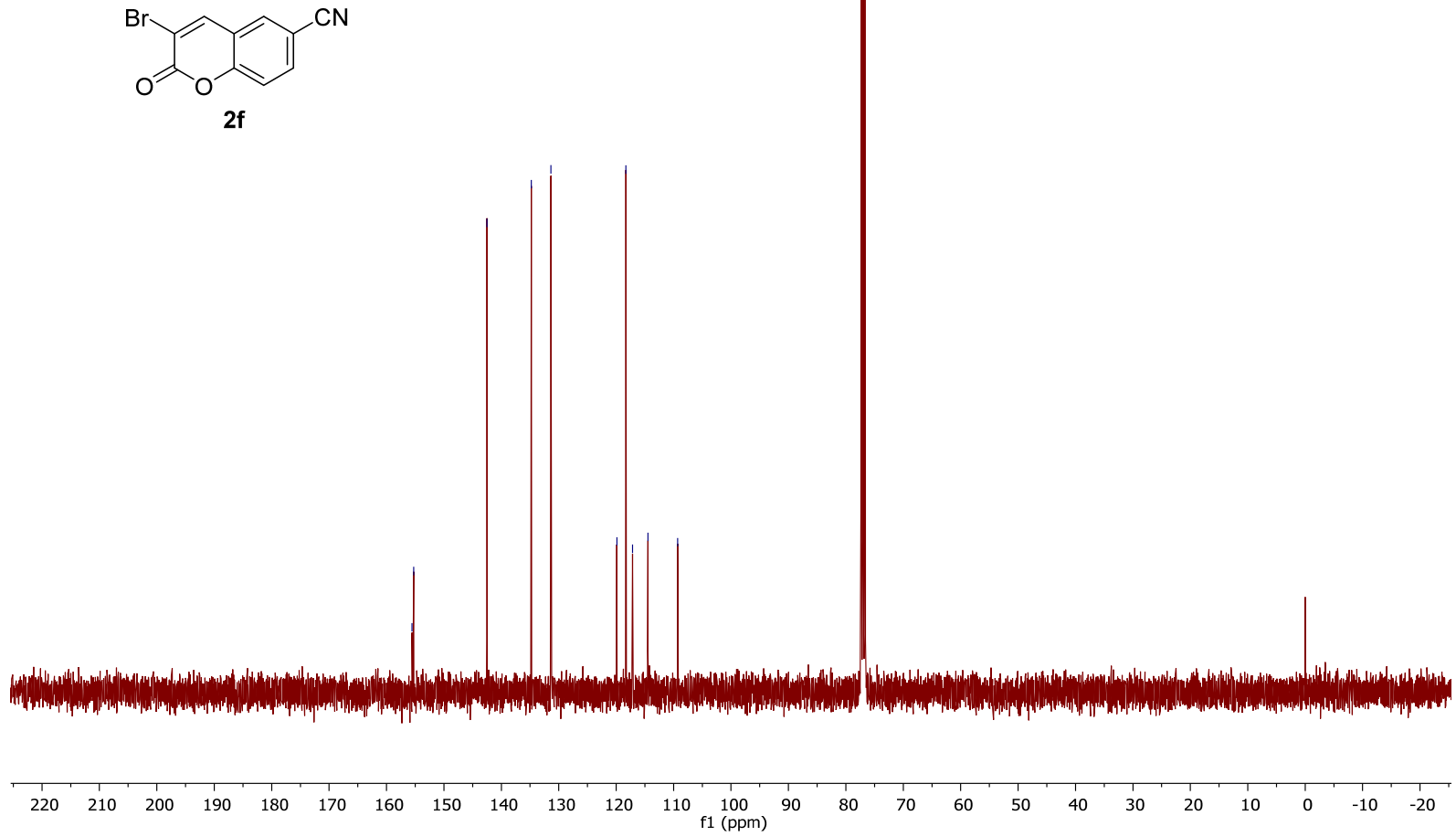

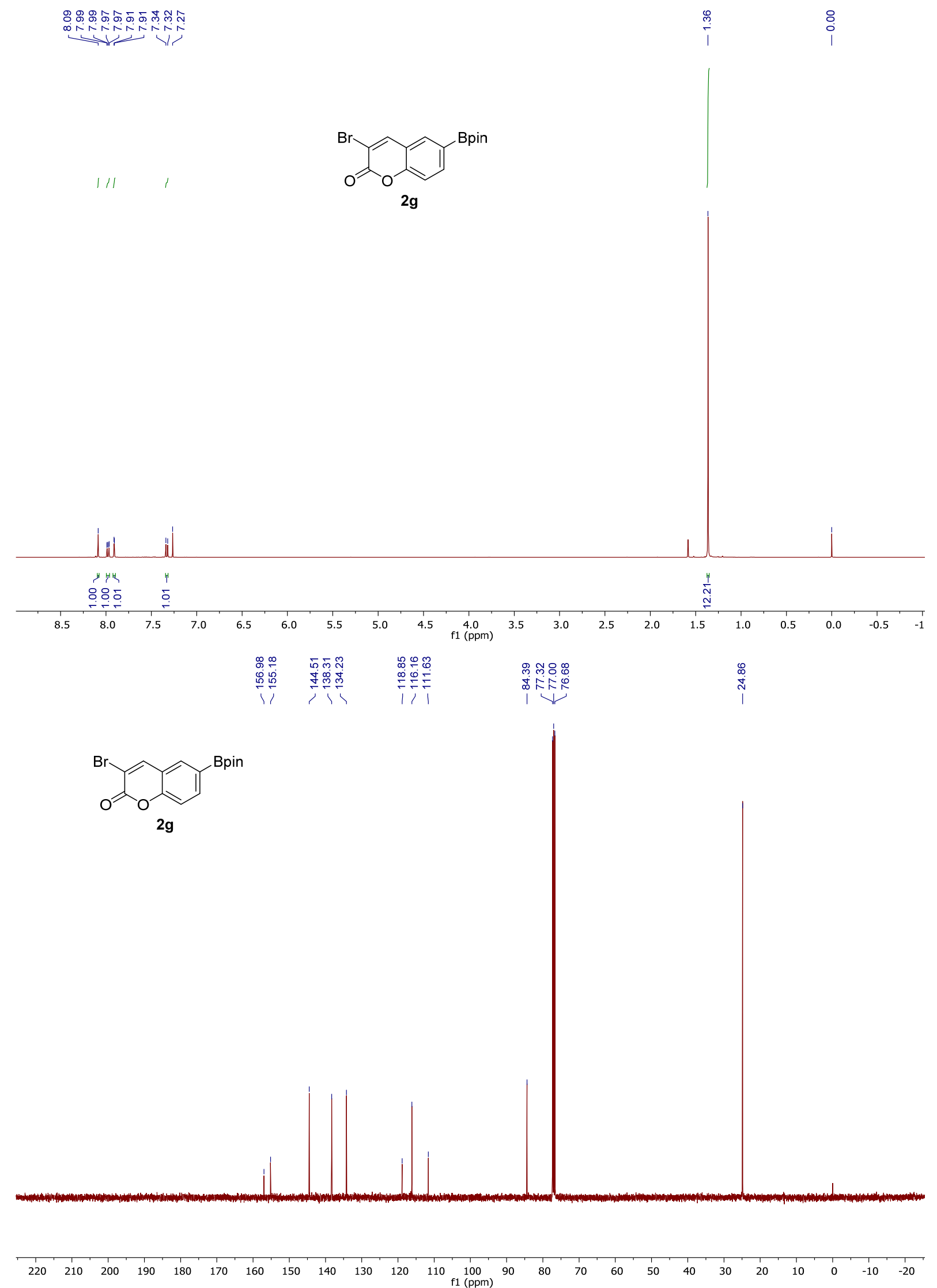


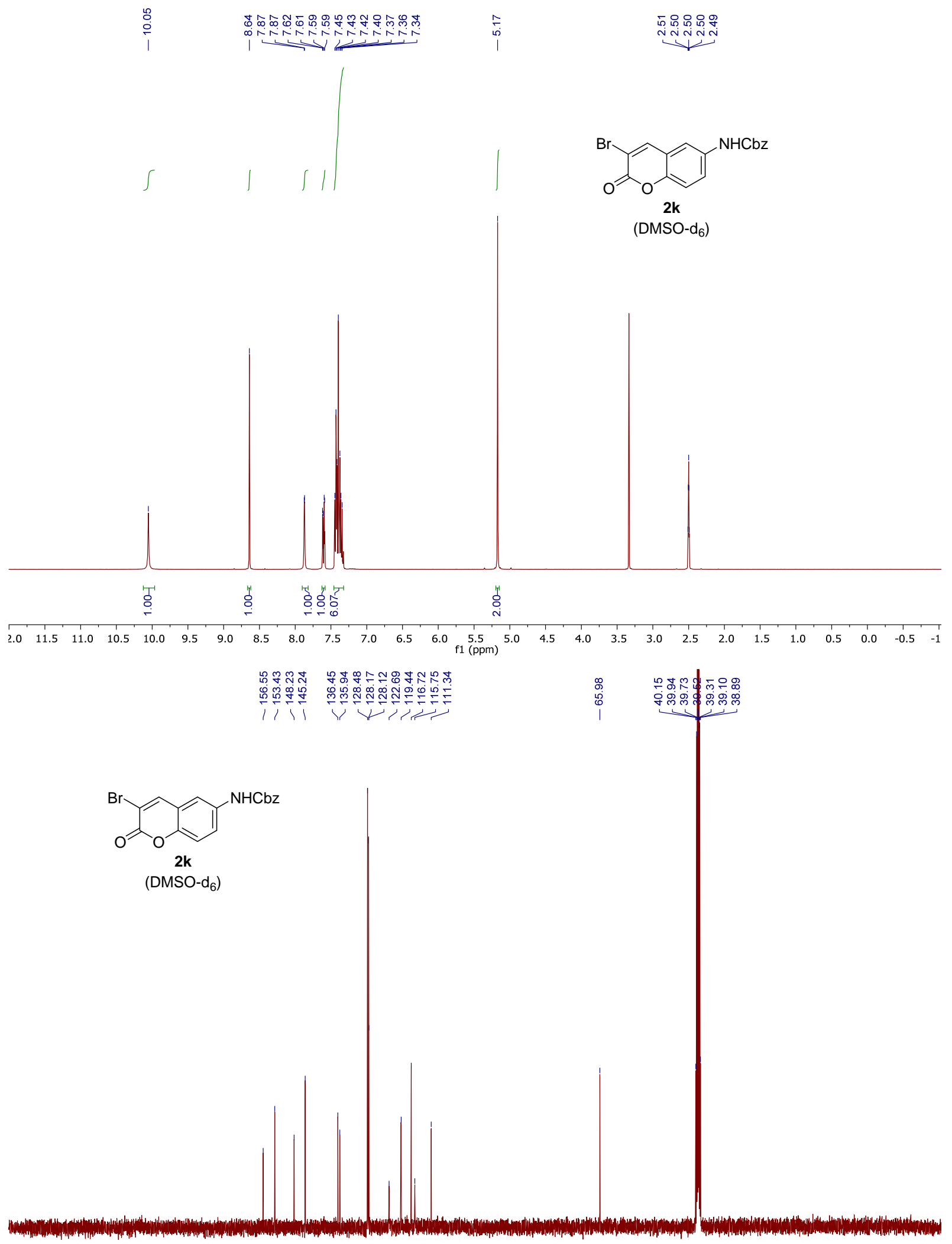

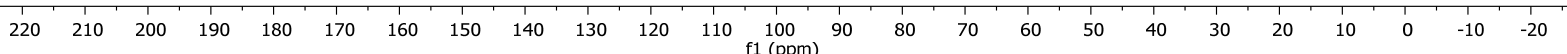




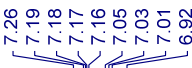

II

$\mathrm{Br}$

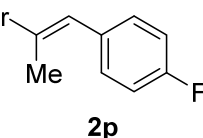

$(E / Z>20: 1)$

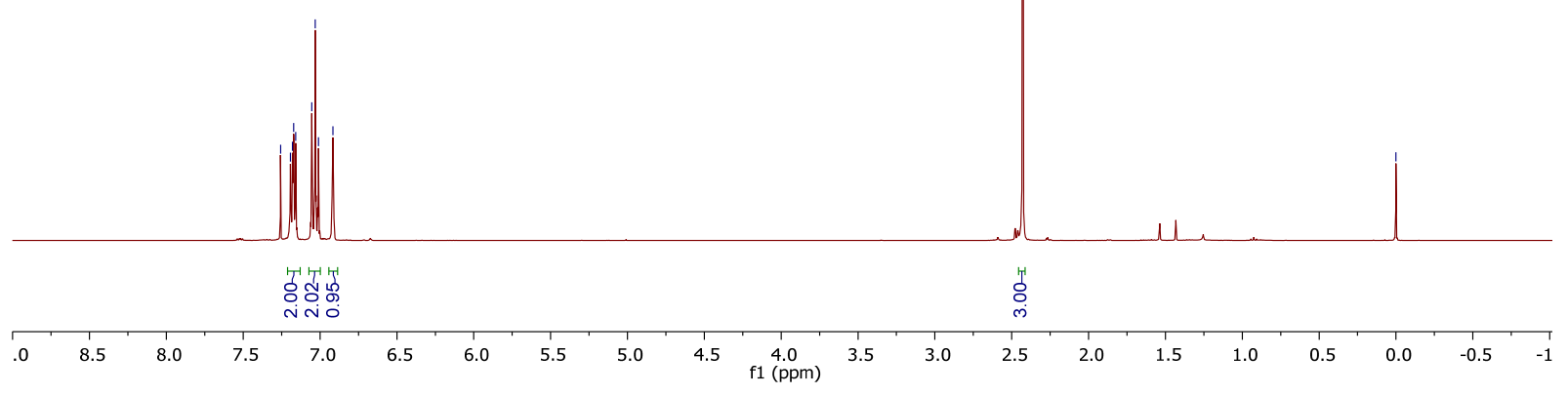

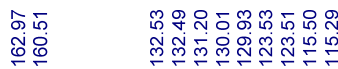

11 岛V

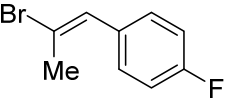

$2 \mathrm{p}$

$(E / Z>20: 1)$

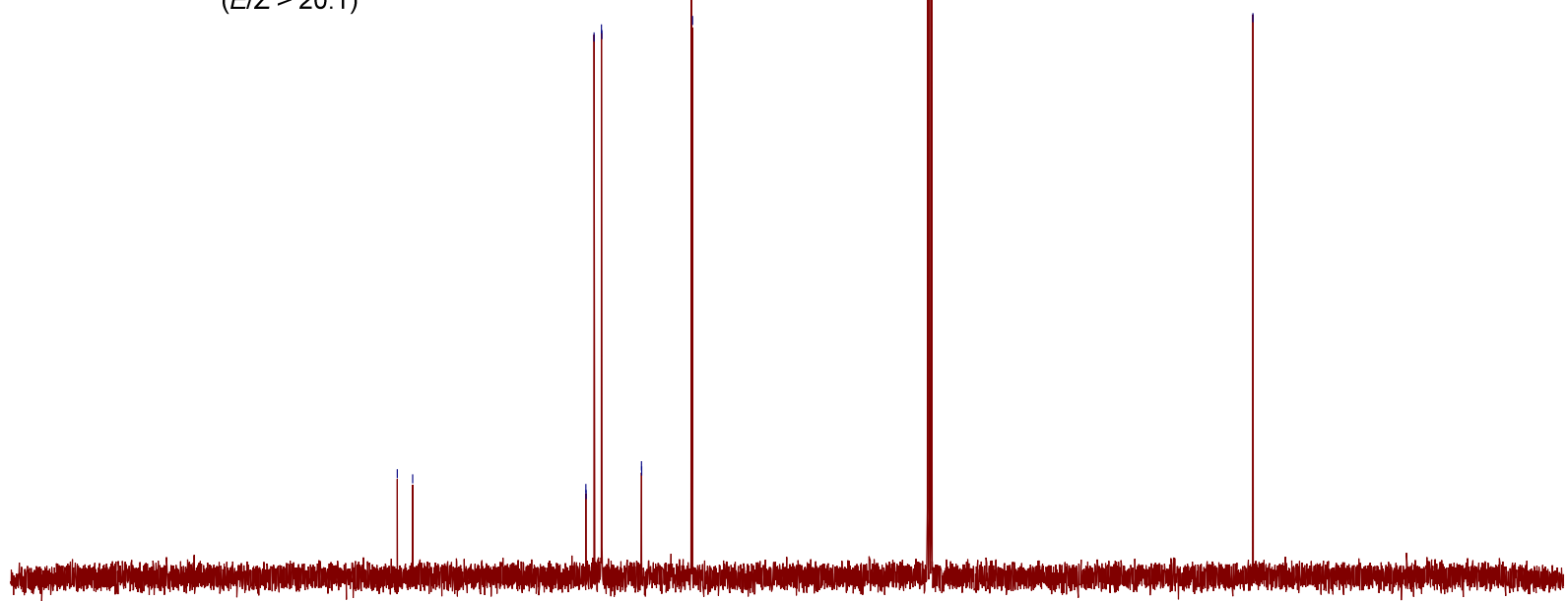

$\begin{array}{lllllllllllllllllllllllllllllllllllll}220 & 210 & 200 & 190 & 180 & 170 & 160 & 150 & 140 & 130 & 120 & 110 & 100 & 90 & 80 & 70 & 60 & 50 & 40 & 30 & 20 & 10 & 0 & -10 & -20\end{array}$ 


\section{${ }^{19}$ F NMR of 2p}

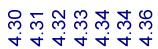
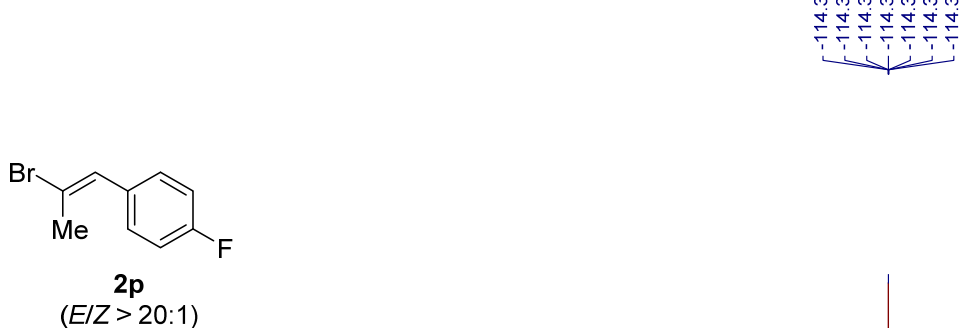

$\begin{array}{llllllllllllllllllllllllll}60 & 50 & 40 & 30 & 20 & 10 & 0 & -10 & -20 & -30 & -40 & -50 & -60 & -70 & -80 & -90 & -100 & -110 & -120 & -130 & -140 & -150 & -160 & -170 & -180\end{array}$ 

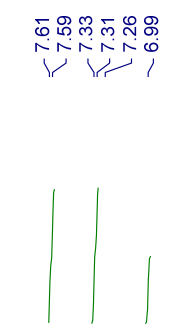

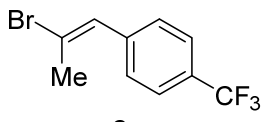

2q

(E/Z>20:1)
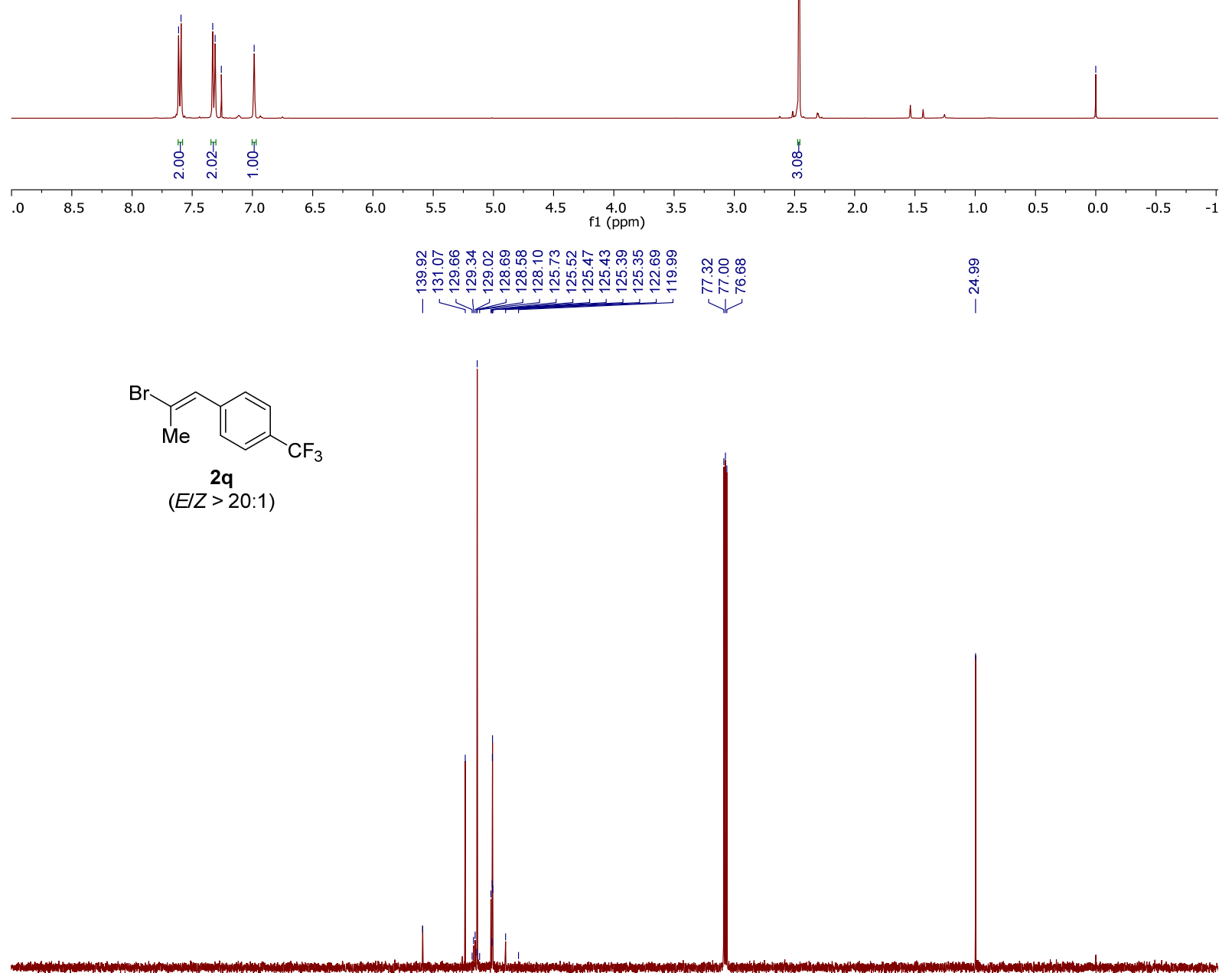

$(E / Z>20: 1)$

$\begin{array}{lllllllllllllllllllllllllllllllllllllll}220 & 210 & 200 & 190 & 180 & 170 & 160 & 150 & 140 & 130 & 120 & 110 & 100 & 90 & 80 & 70 & 60 & 50 & 40 & 30 & 20 & 10 & 0 & -10 & -20\end{array}$ 
${ }^{19}$ F NMR of 2q
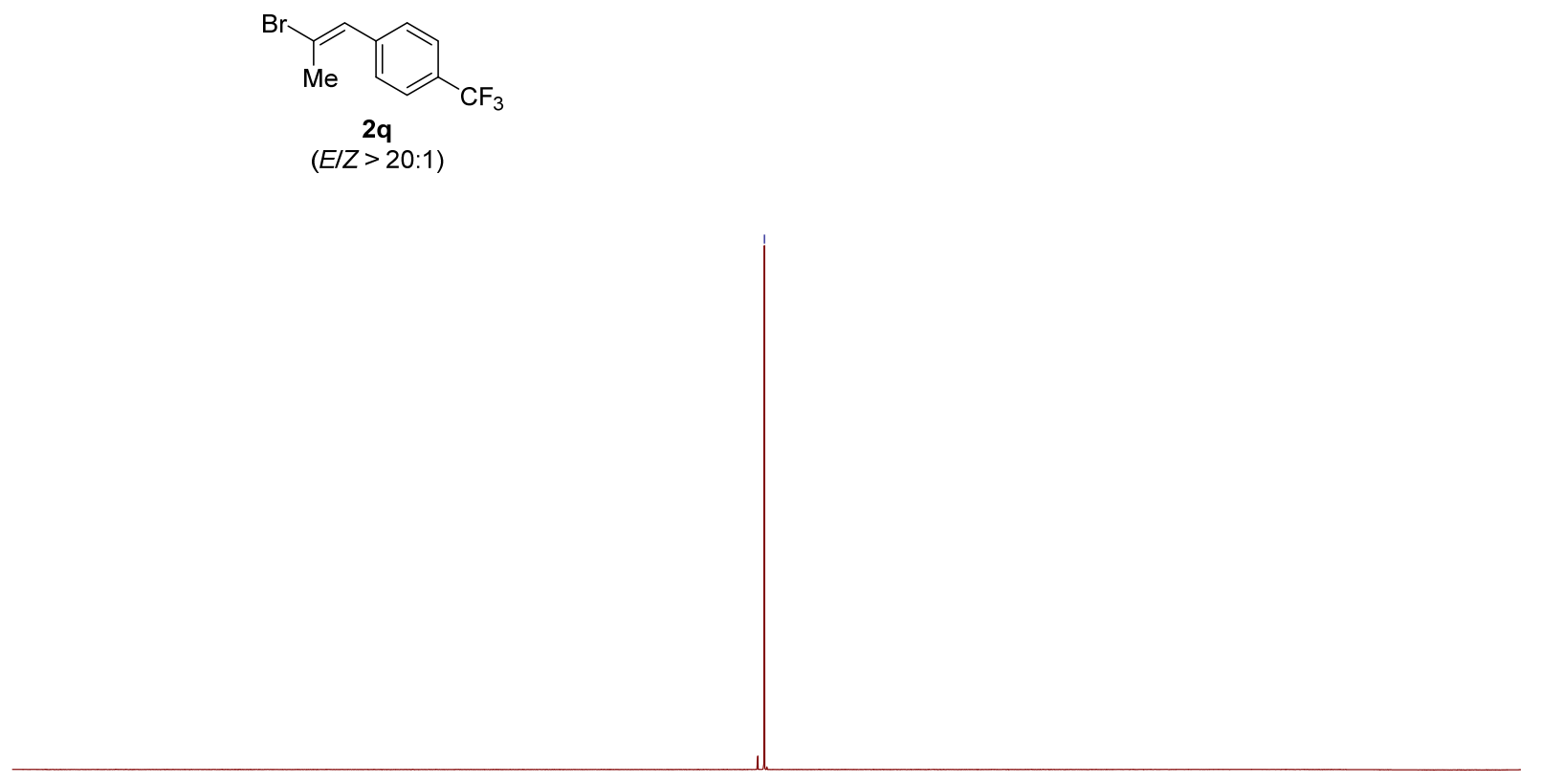

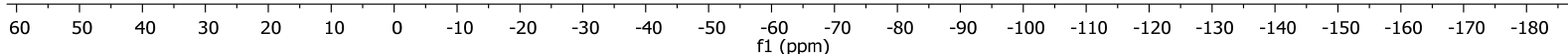



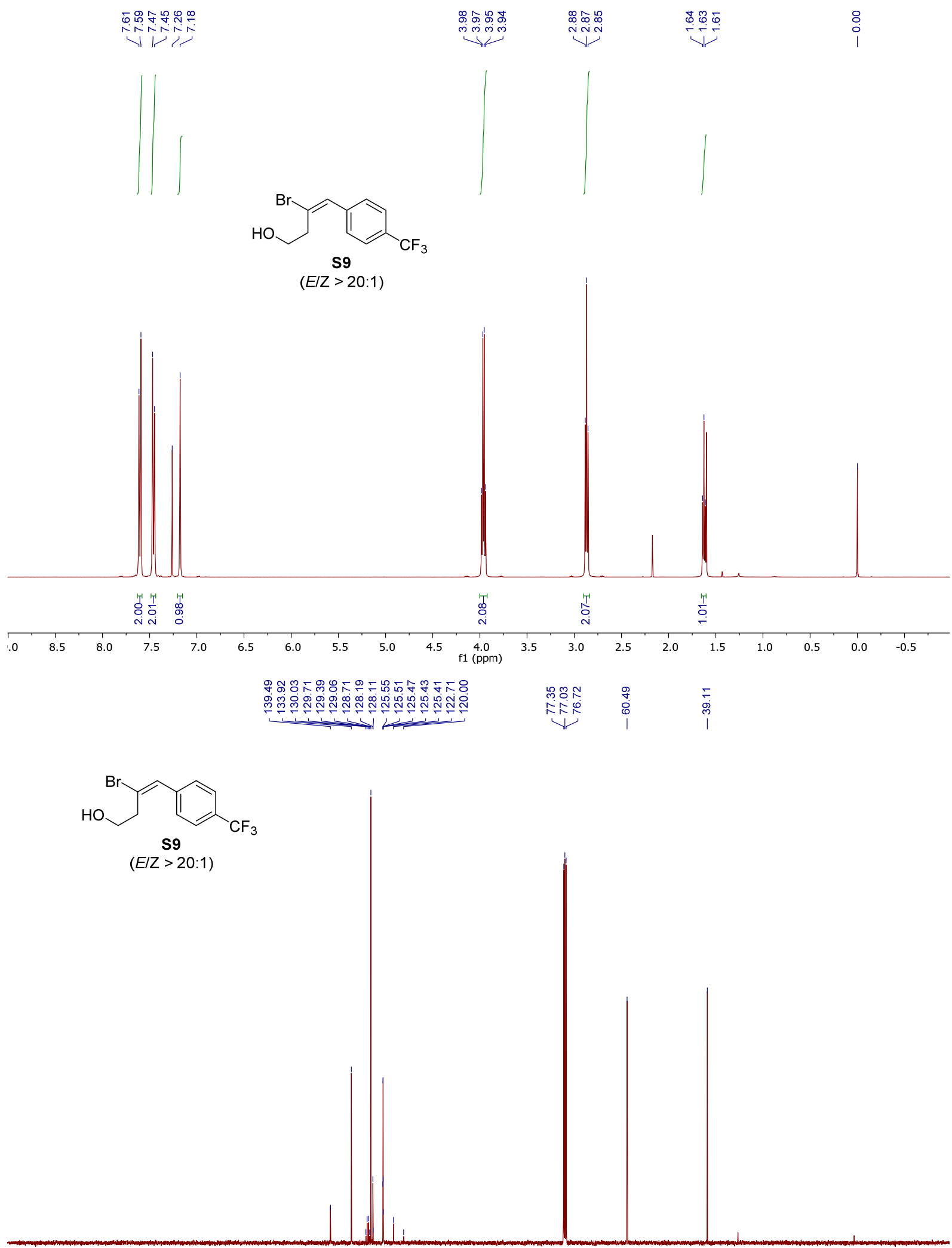

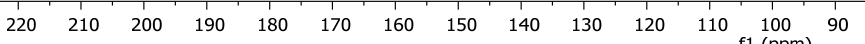
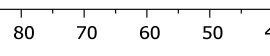


\section{${ }^{19}$ F NMR of S9}

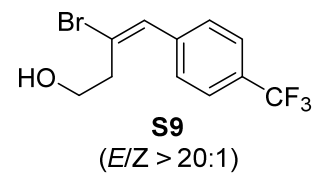

$\begin{array}{llllllllllllllllllllllllll}60 & 50 & 40 & 30 & 20 & 10 & 0 & -10 & -20 & -30 & -40 & -50 & -60 & -70 & -80 & -90 & -100 & -110 & -120 & -130 & -140 & -150 & -160 & -170 & -180\end{array}$ 

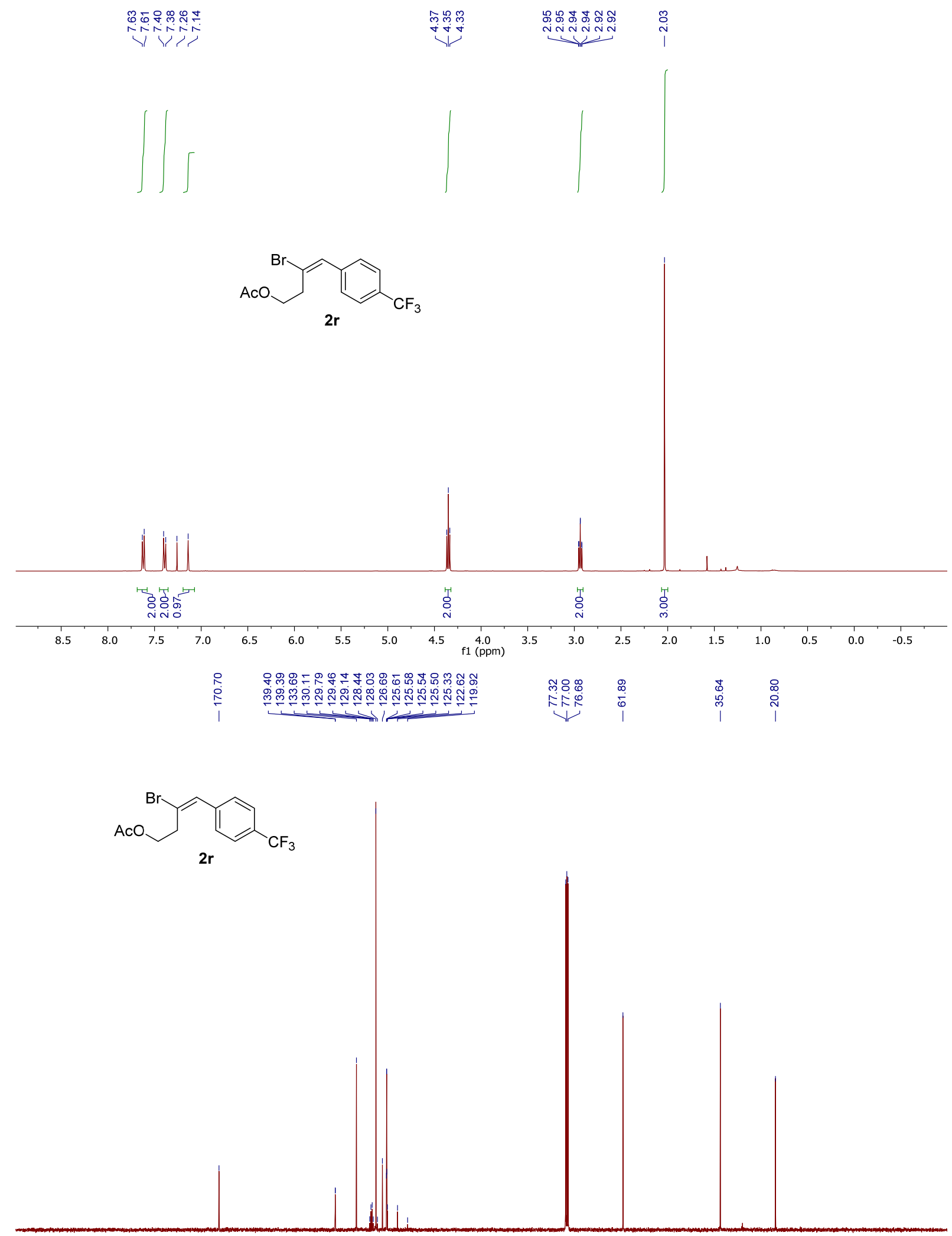

$\begin{array}{lllllllllllllllllllllllllll}\mid & 220 & 210 & 200 & 190 & 180 & 170 & 160 & 150 & 140 & 130 & 120 & 110 & 100 & 90 & 80 & 70 & 60 & 50 & 40 & 30 & 20 & 10 & 0 & -10 & -20\end{array}$ 


\section{${ }^{19}$ F NMR of $2 r$}<smiles>CC(=O)OCC/C(Br)=C\c1ccc(C(F)(F)F)cc1</smiles>

$\begin{array}{lllllllllllllllllllllllllllll} & 60 & 50 & 40 & 30 & 20 & 10 & 0 & -10 & -20 & -30 & -40 & -50 & -60 & -70 & -80 & -90 & -100 & -110 & -120 & -130 & -140 & -150 & -160 & -170 & -180\end{array}$ 

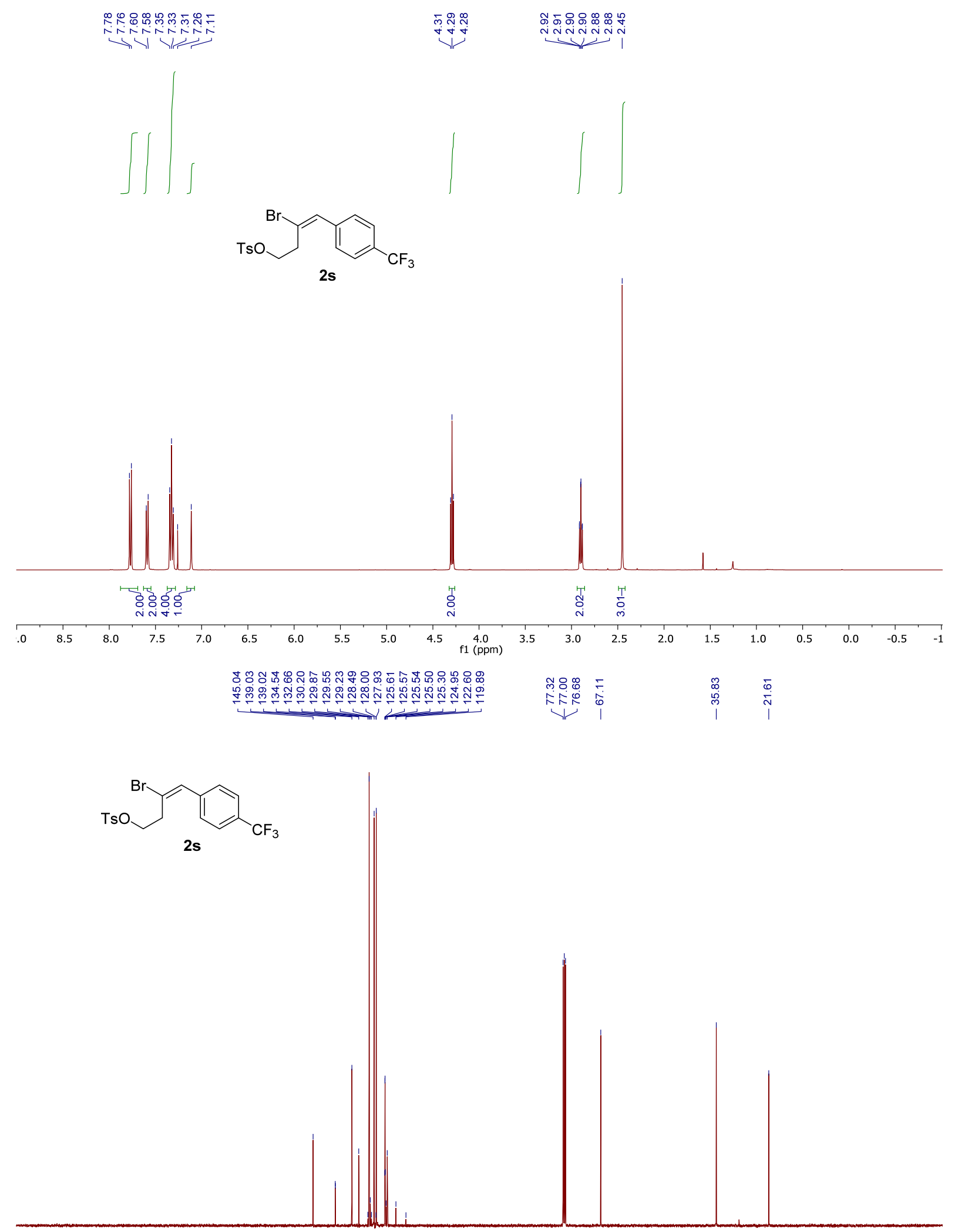

$\begin{array}{lllllllllllllllllllllllllllllllllllllll}220 & 210 & 200 & 190 & 180 & 170 & 160 & 150 & 140 & 130 & 120 & 110 & 100 & 90 & 80 & 70 & 60 & 50 & 40 & 30 & 20 & 10 & 0 & -10 & -20\end{array}$ 
${ }^{19}$ F NMR of $2 \mathrm{~s}$

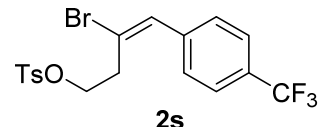

2s

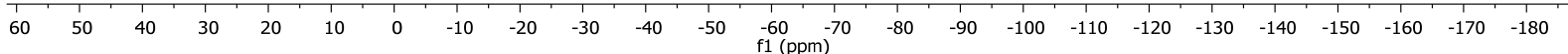




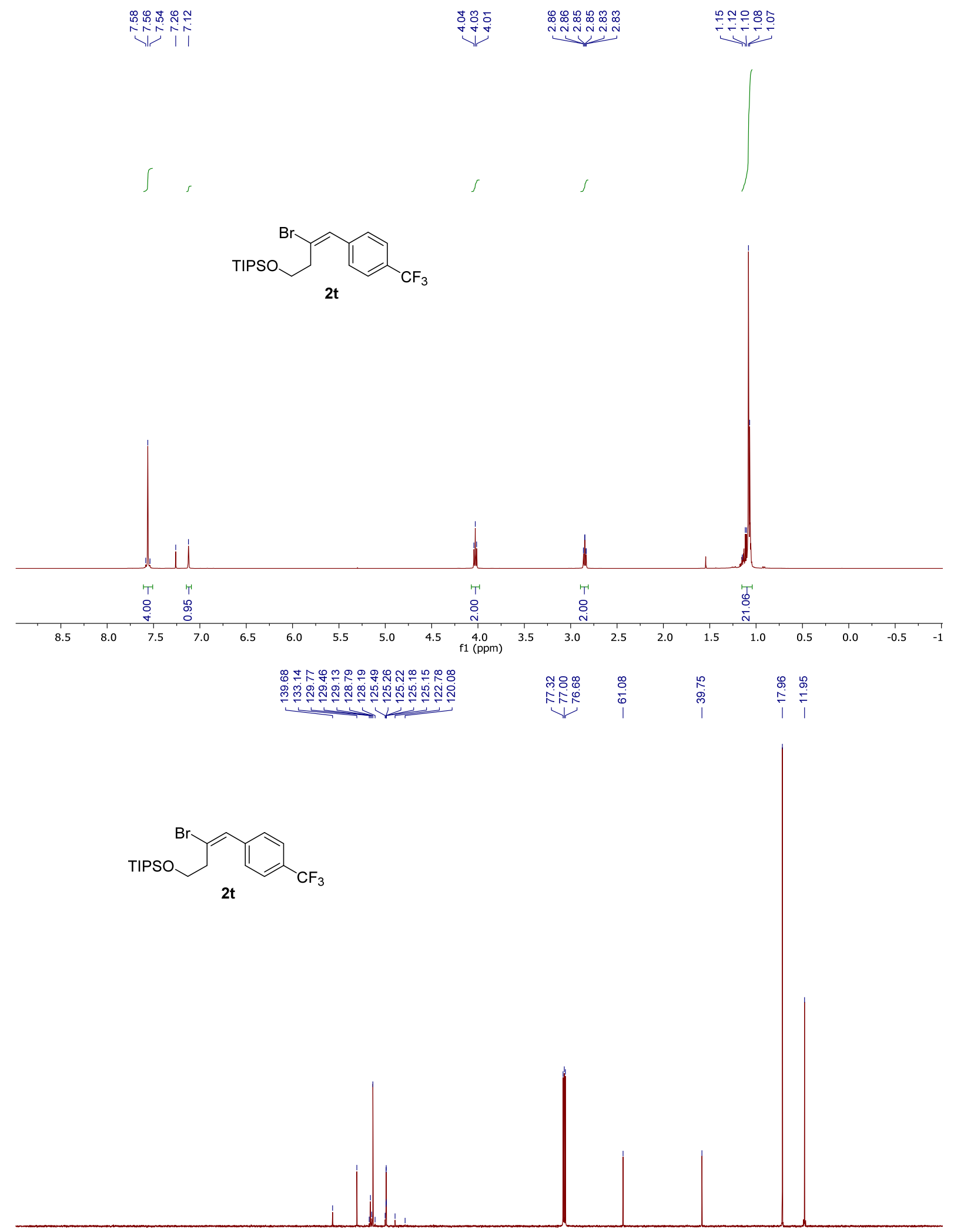

$\begin{array}{llllllllllllllllllllllllll}\mid & 220 & 210 & 200 & 190 & 180 & 170 & 160 & 150 & 140 & 130 & 120 & 110 & 100 & 90 & 80 & 70 & 60 & 50 & 40 & 30 & 20 & 10 & 0 & -10 & -20\end{array}$ 
${ }^{19}$ F NMR of $2 t$

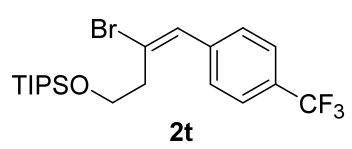

$\begin{array}{lllllllllllllllllllllllllllllll}7 & 60 & 50 & 40 & 30 & 20 & 10 & 0 & -10 & -20 & -30 & -40 & -50 & -60 & -70 & -80 & -90 & -100 & -110 & -120 & -130 & -140 & -150 & -160 & -170 & -180\end{array}$ 


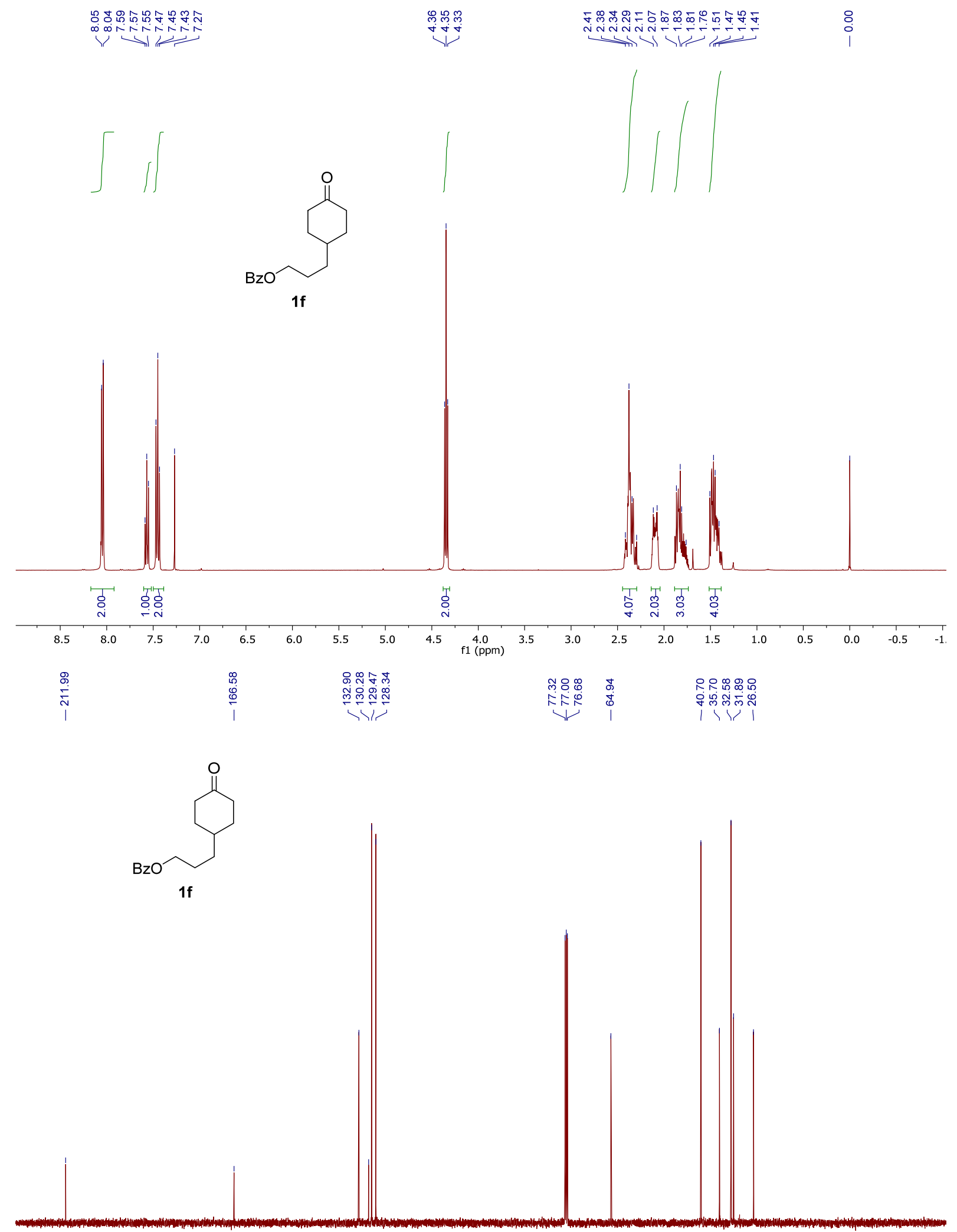

BzO

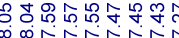

i

i

Y

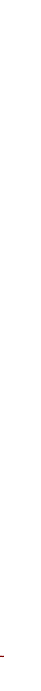



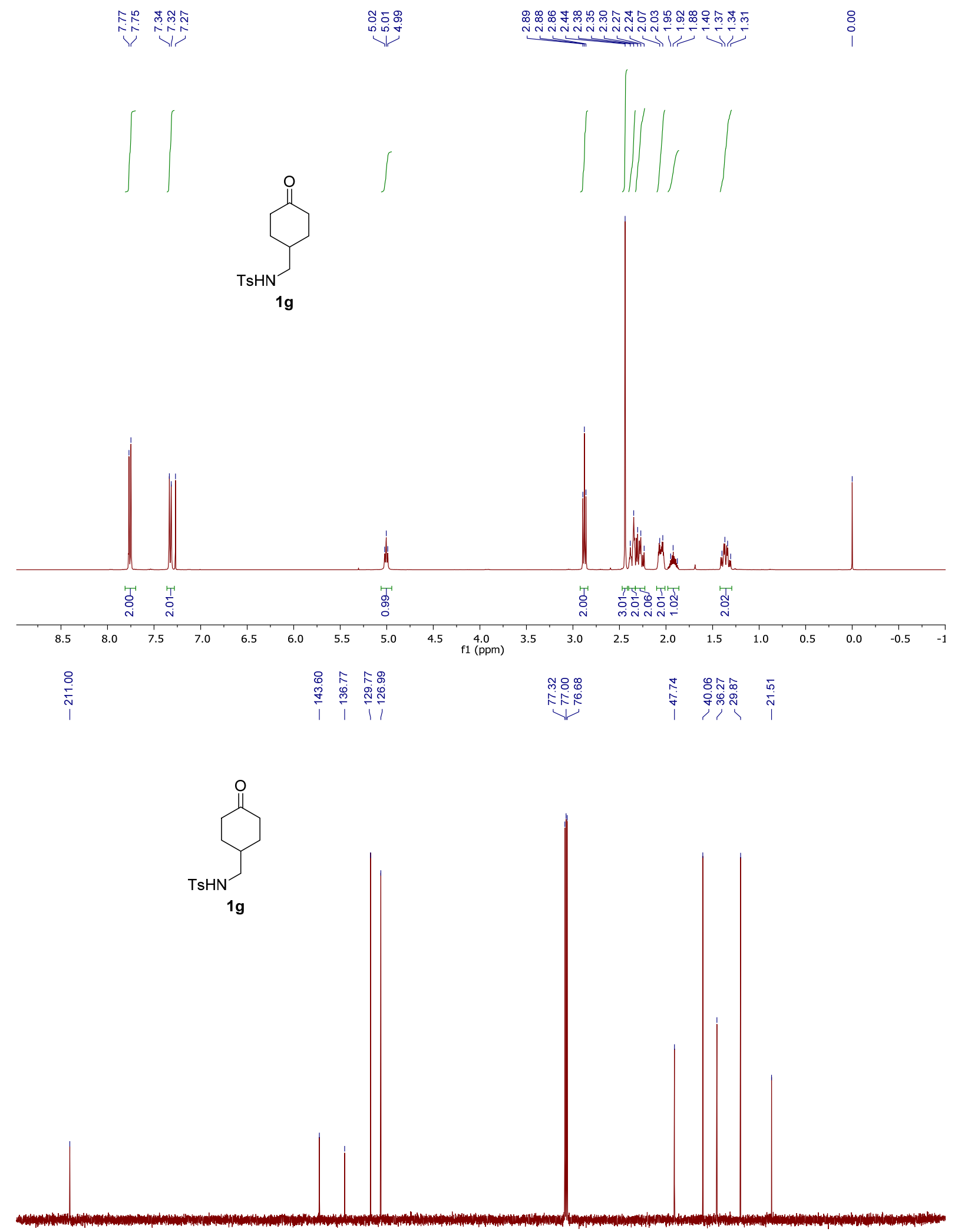

$\begin{array}{llllllllllllllllllllllllllllllllllllll}220 & 210 & 200 & 190 & 180 & 170 & 160 & 150 & 140 & 130 & 120 & 110 & 100 & 90 & 80 & 70 & 60 & 50 & 40 & 30 & 20 & 10 & 0 & -10 & -20\end{array}$ 


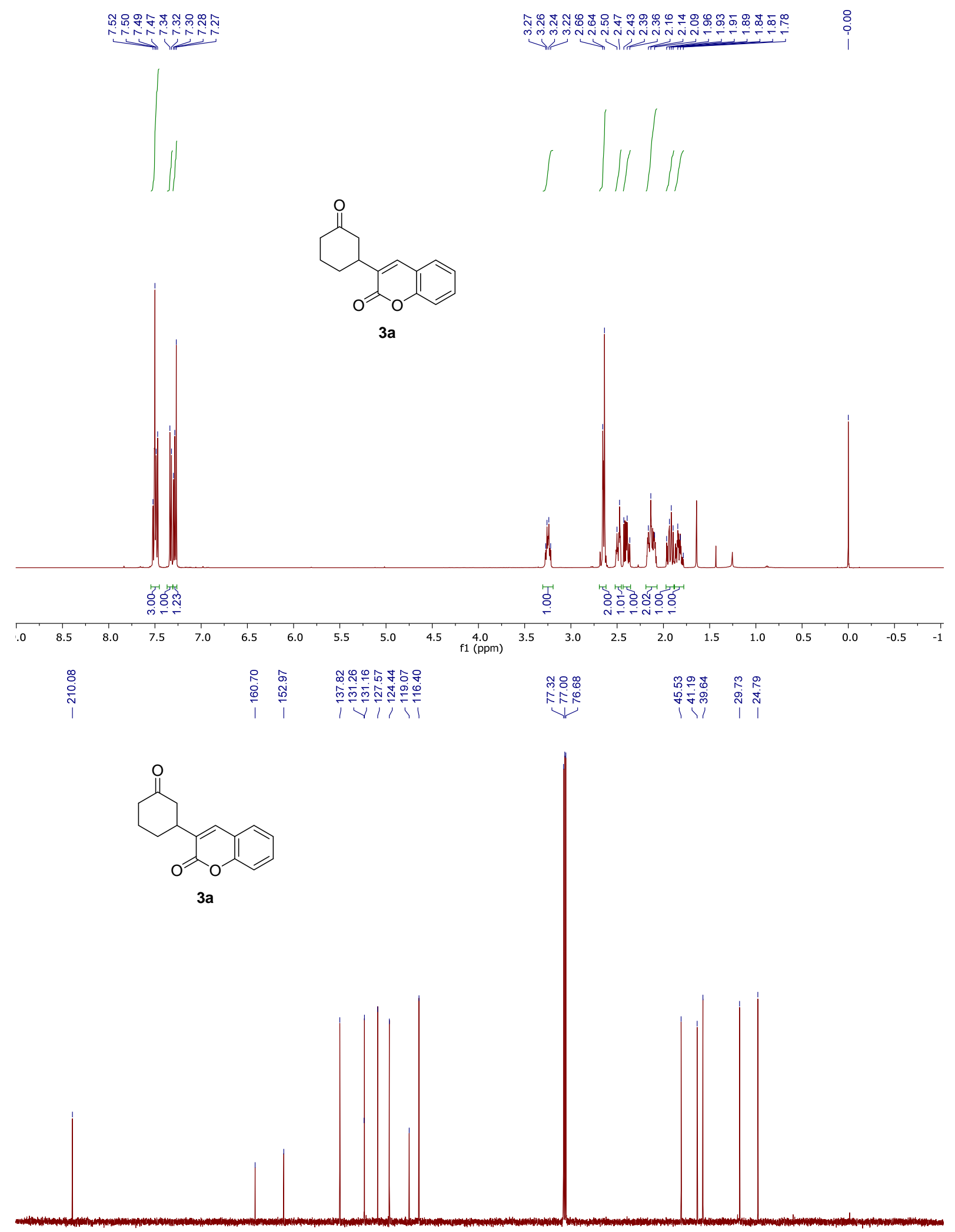

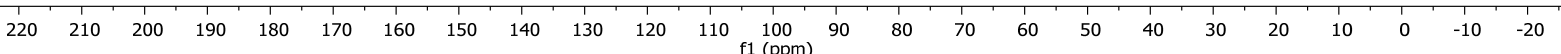




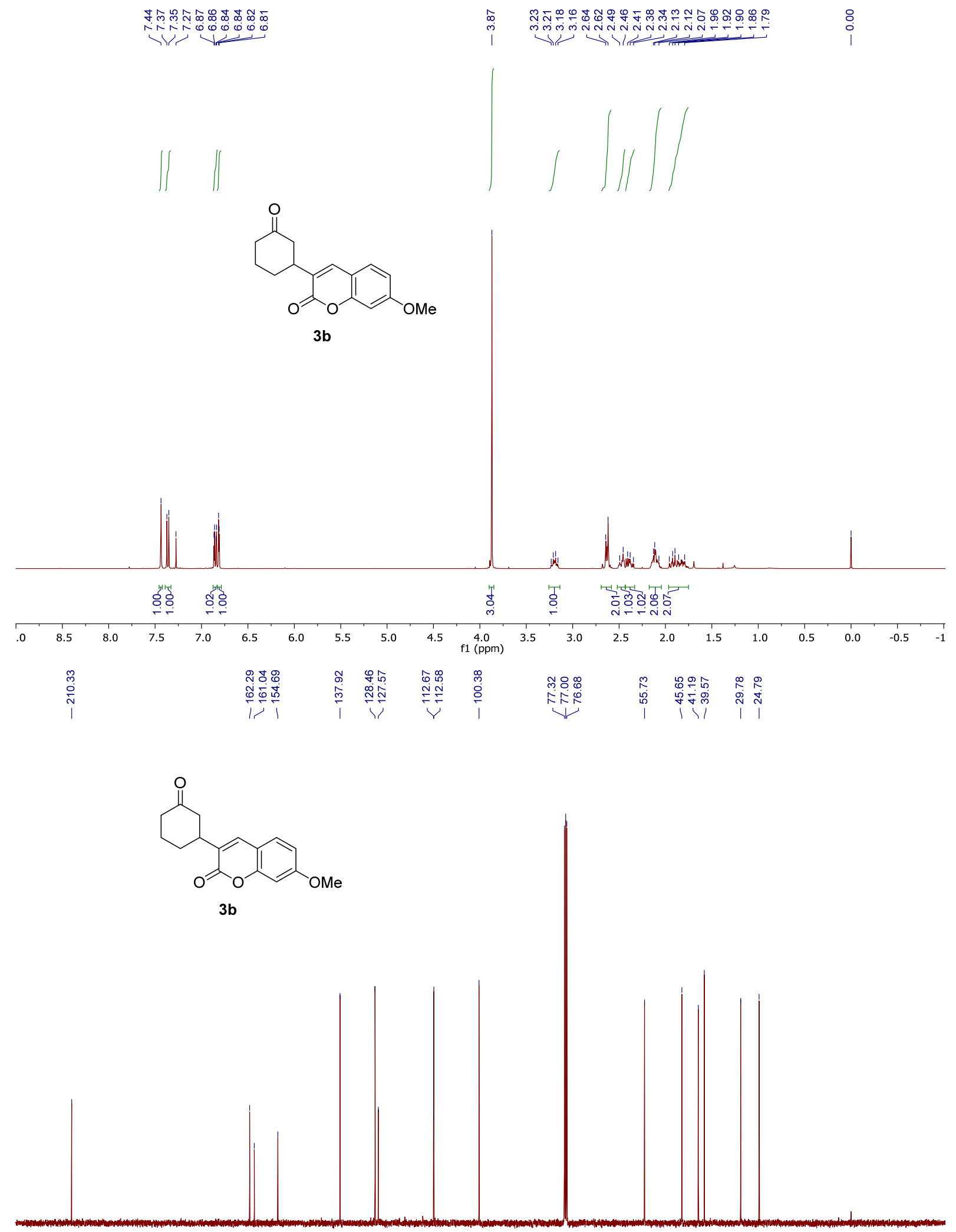

(1)

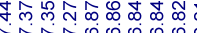

讨

| N⿴囗十

$3 b$ 


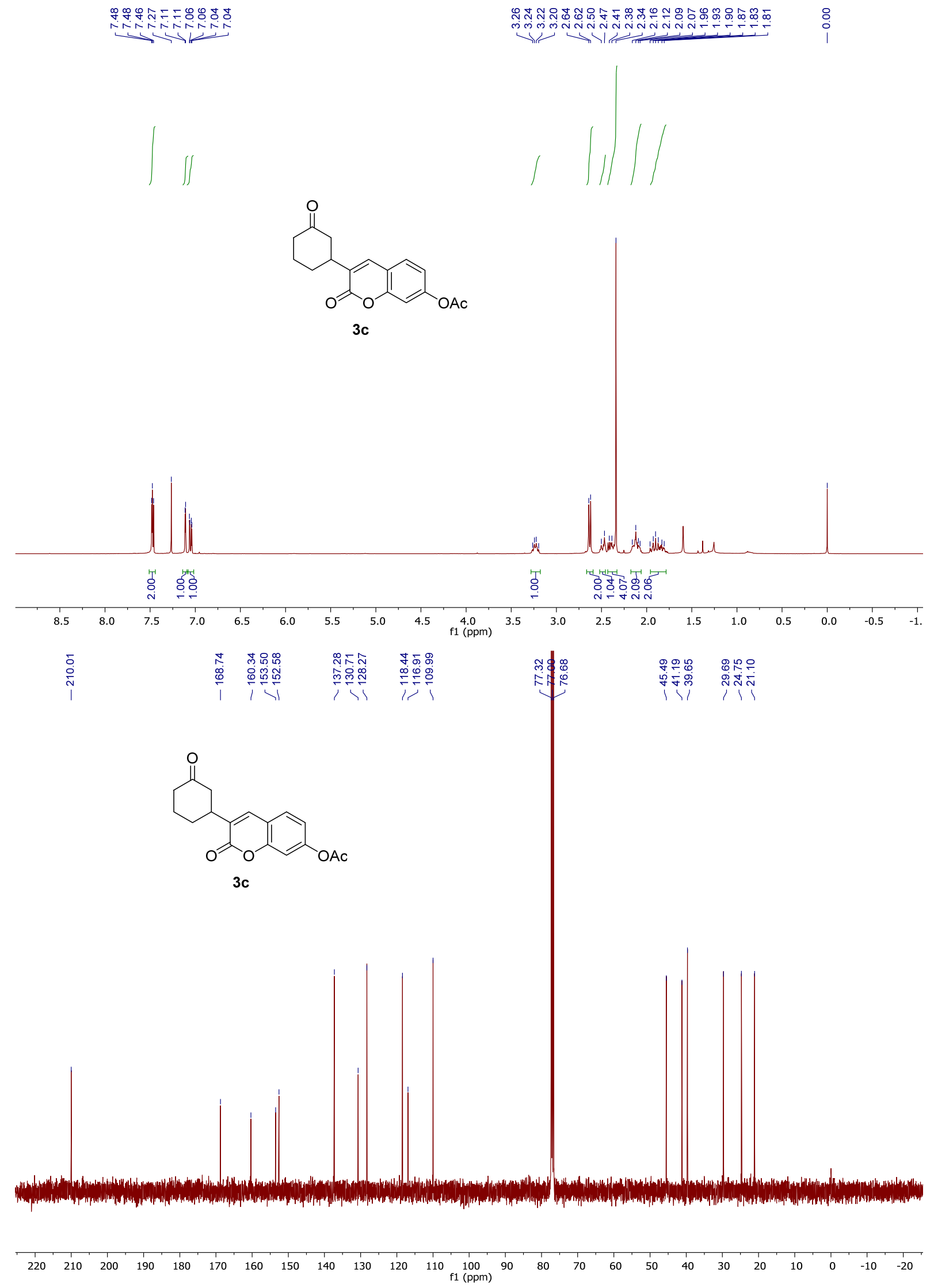




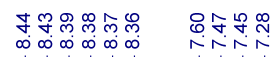

în

$\iiint$

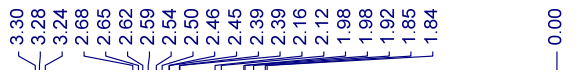

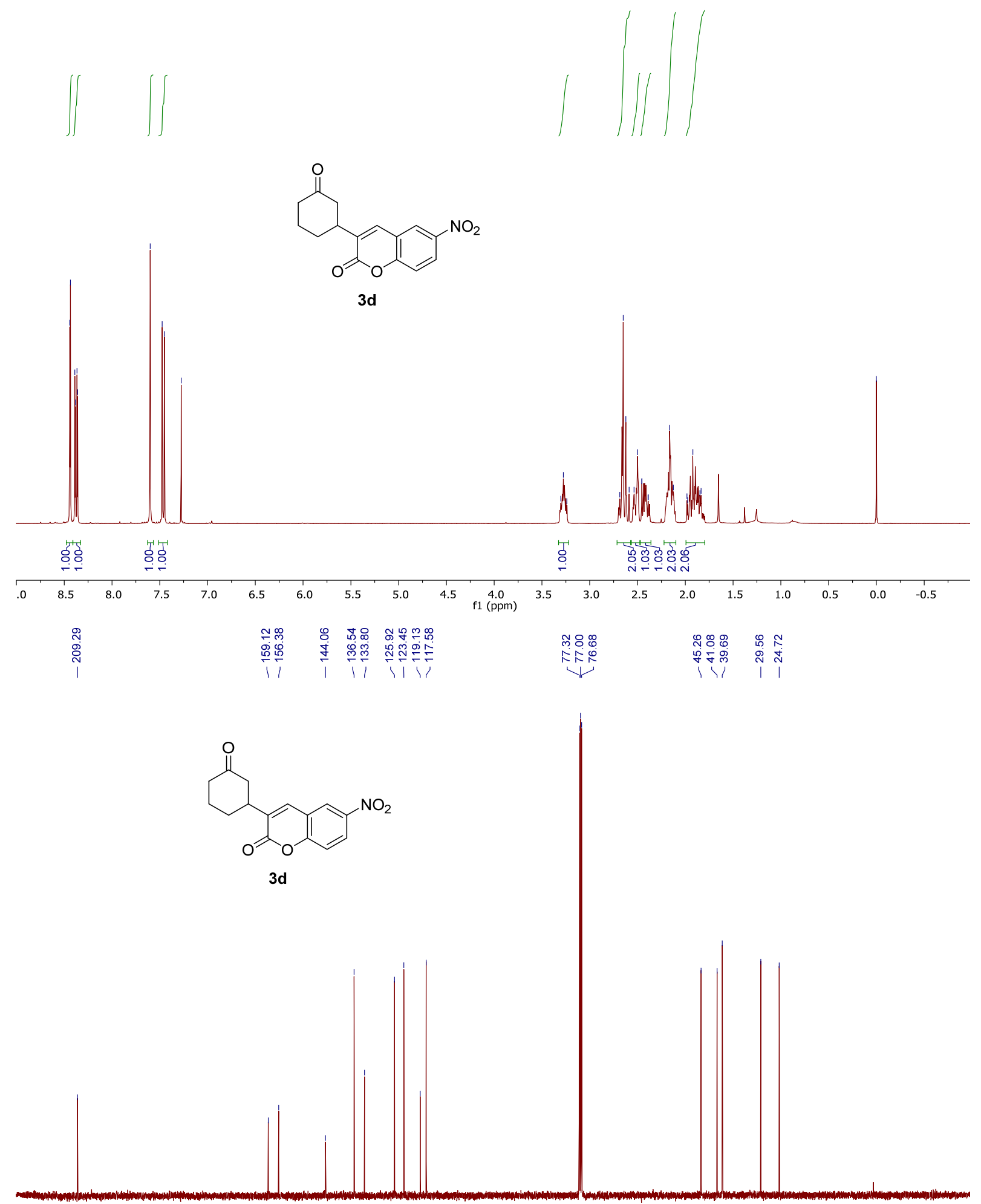

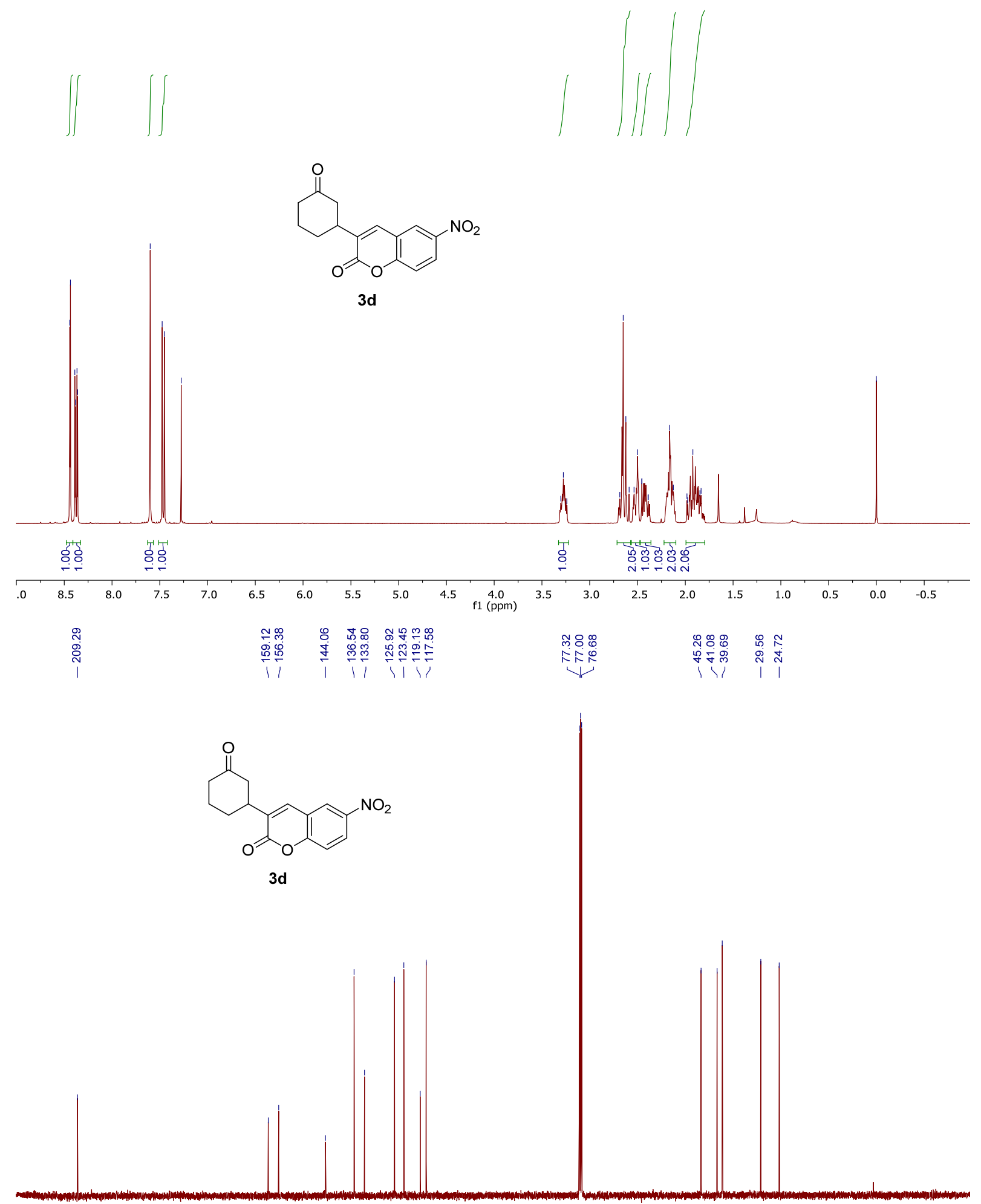

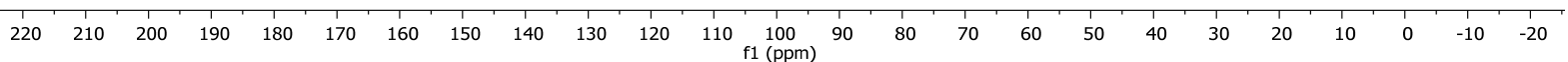


$\overline{2} \bar{\alpha}$

我

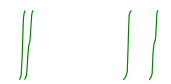

$3 e$

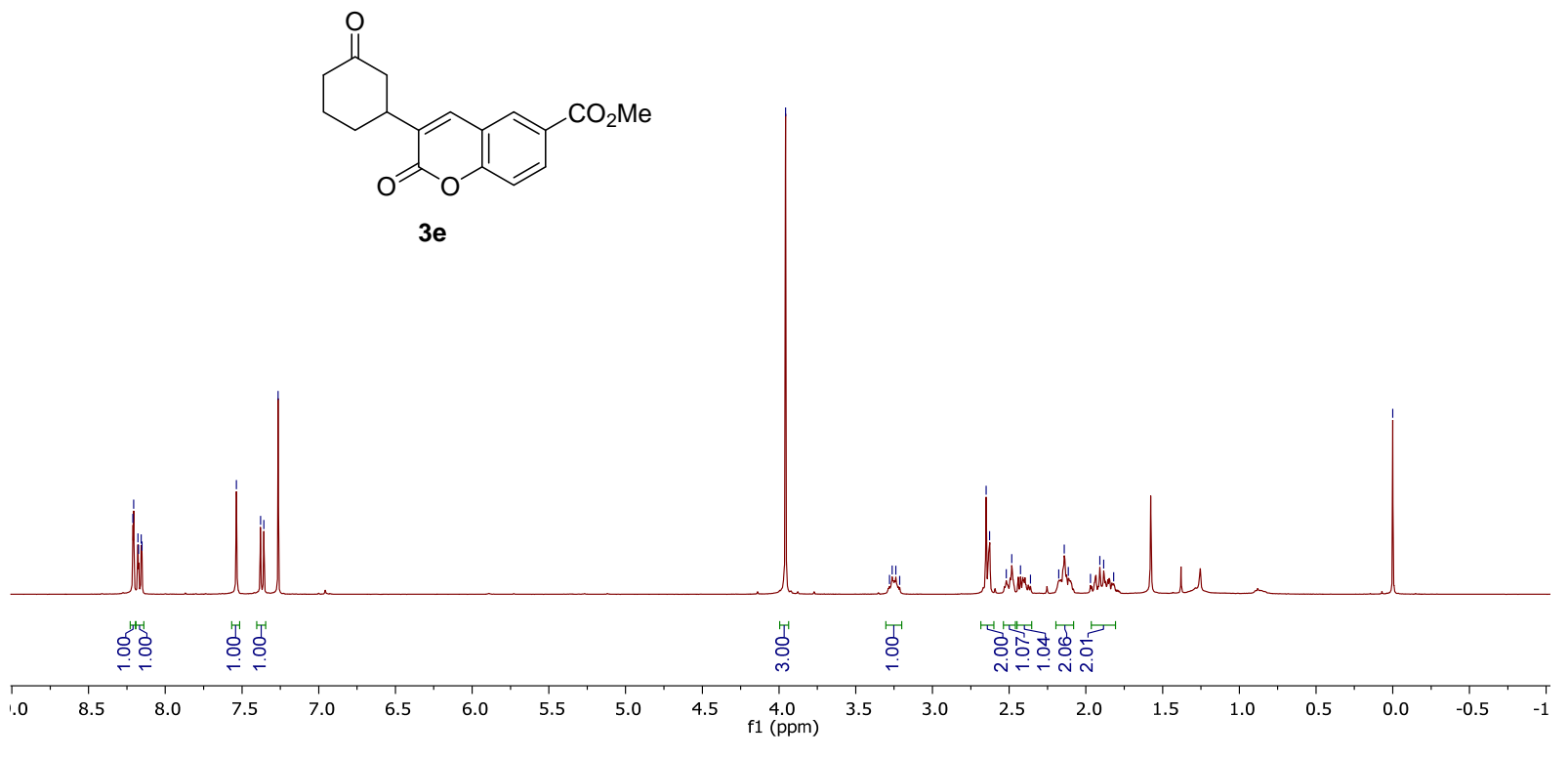

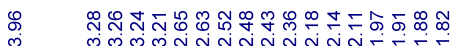

Mmm NNNNNNNN

级

$\stackrel{8}{8}$

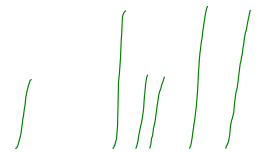

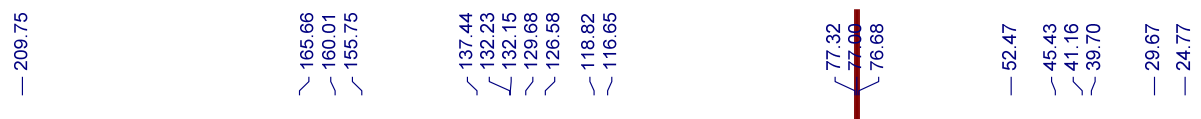

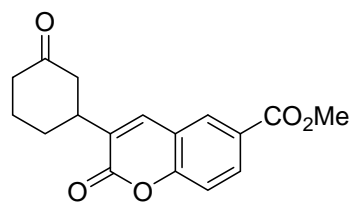

$3 e$

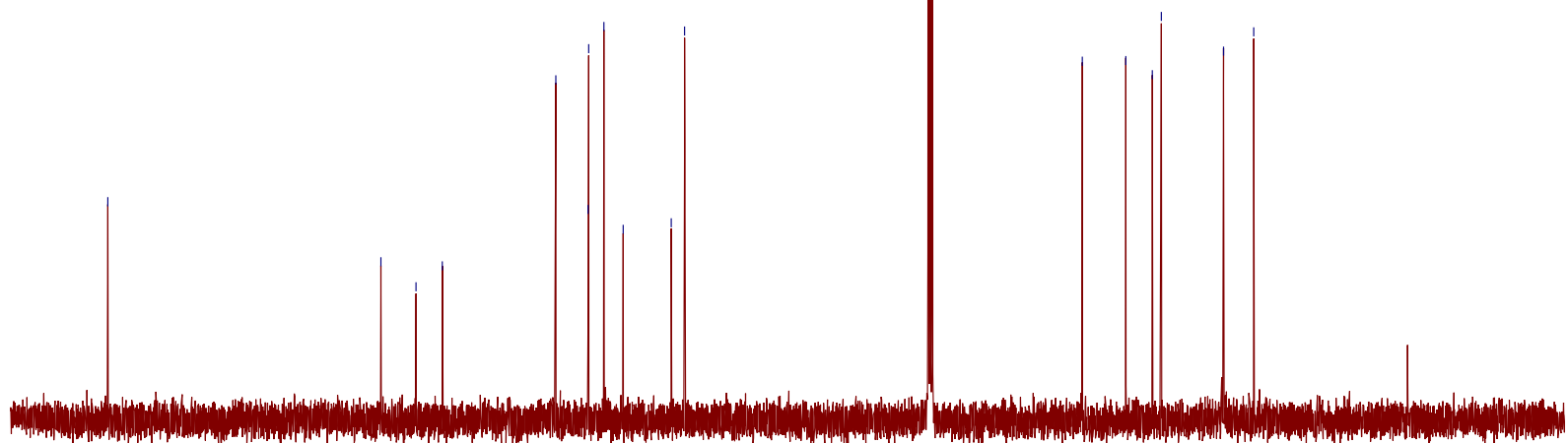

$\begin{array}{lllllllllllllllllllllllllllllllllllll}220 & 210 & 200 & 190 & 180 & 170 & 160 & 150 & 140 & 130 & 120 & 110 & 100 & 90 & 80 & 70 & 60 & 50 & 40 & 30 & 20 & 10 & 0 & -10 & -20\end{array}$ 


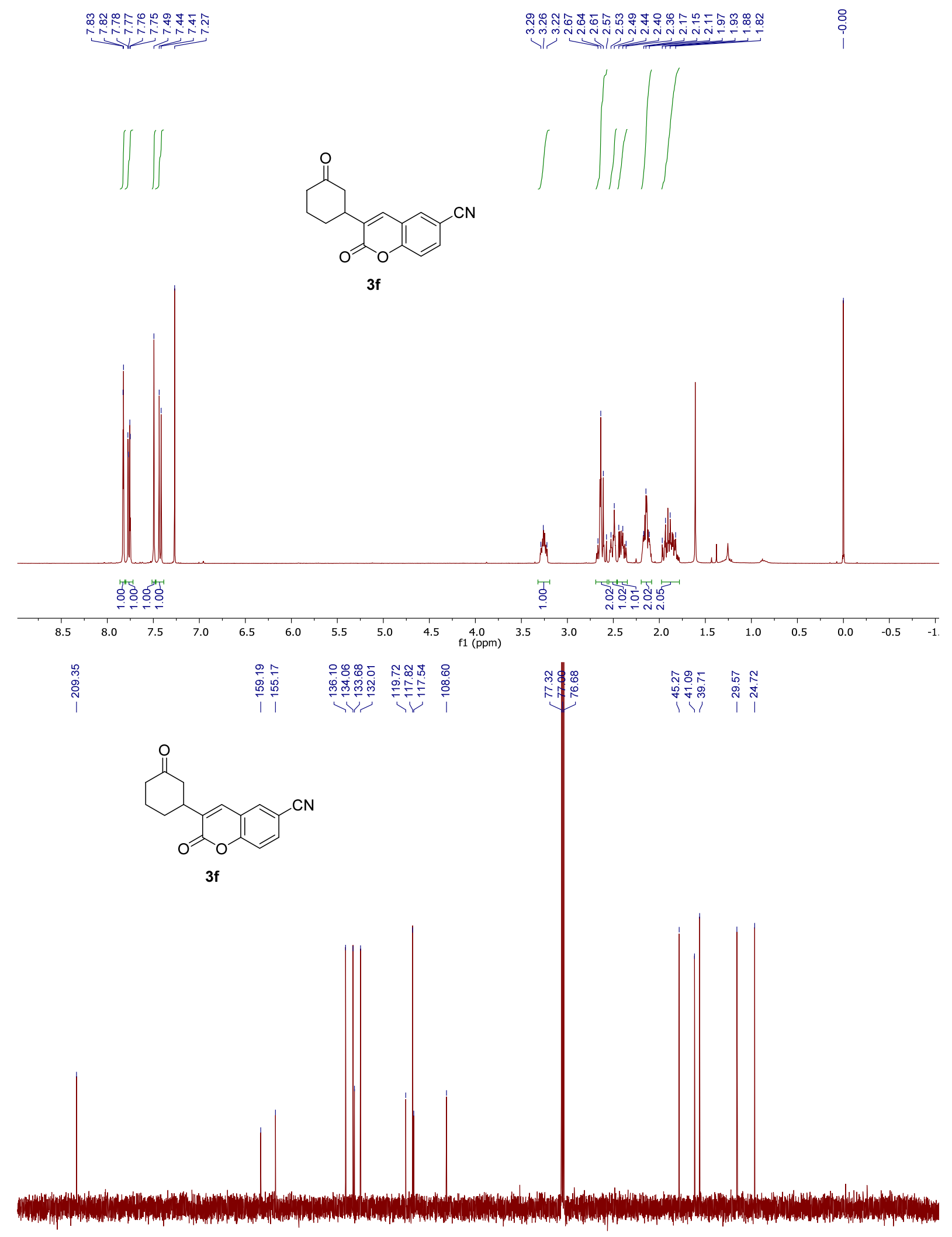

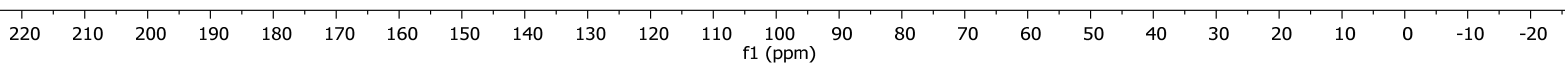




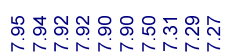

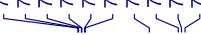

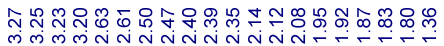

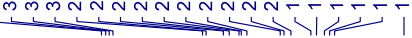

웅<smiles>O=C1CCCC(c2cc3cc(Cc4ccccc4)ccc3oc2=O)C1</smiles>

$3 g$

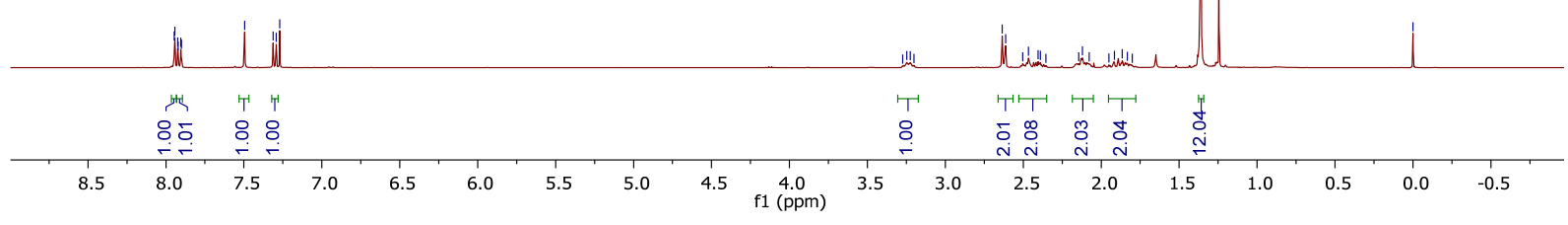

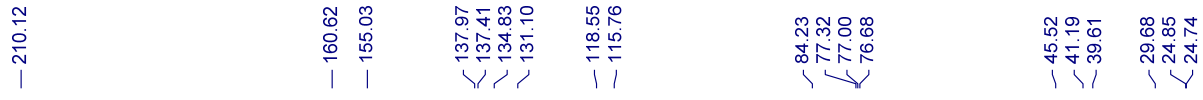<smiles>O=C1CCCC(c2cc3cc(Cc4ccccc4)ccc3oc2=O)C1</smiles>

$3 \mathrm{~g}$

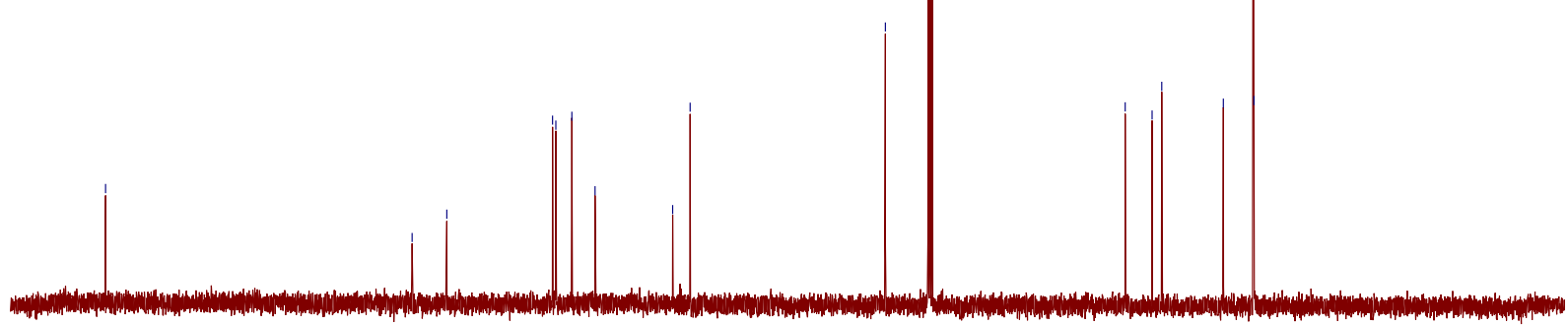

$\begin{array}{llllllllllllll}220 & 210 & 200 & 190 & 180 & 170 & 160 & 150 & 140 & 130 & 120 & 110 & 100 & 90\end{array}$

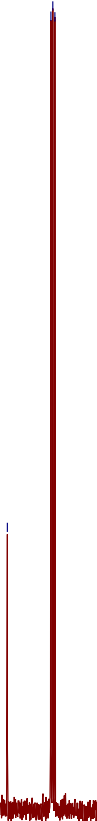



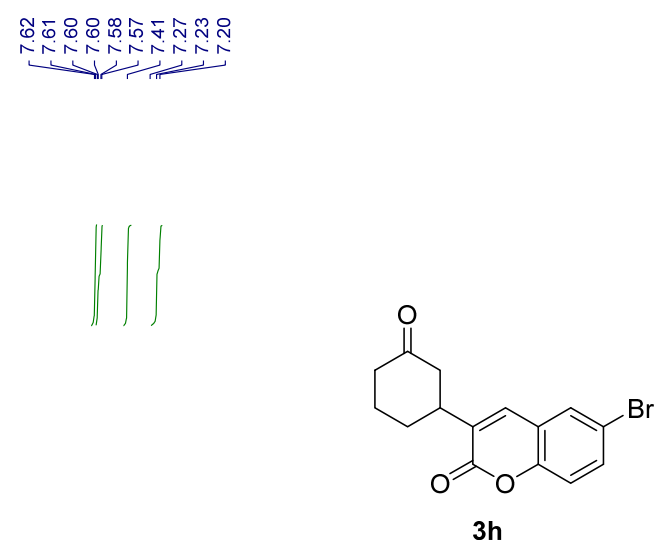

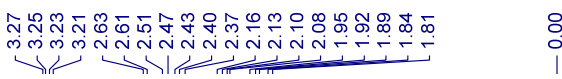
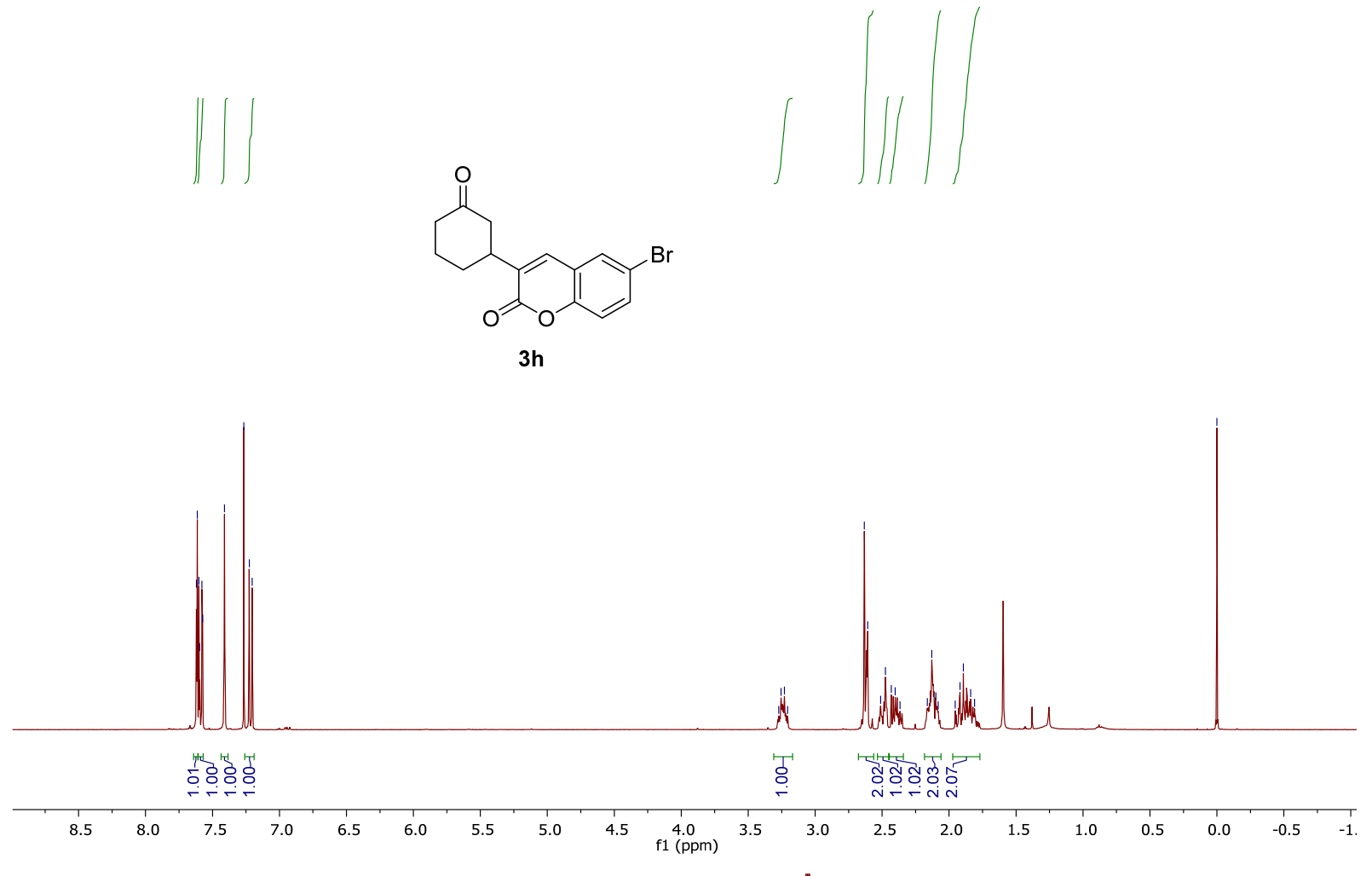

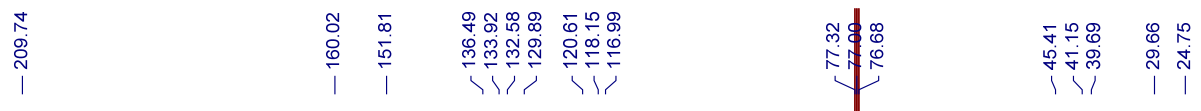<smiles>O=C1CCCC(c2cc3cc(Br)ccc3oc2=O)C1</smiles>

$3 \mathrm{~h}$

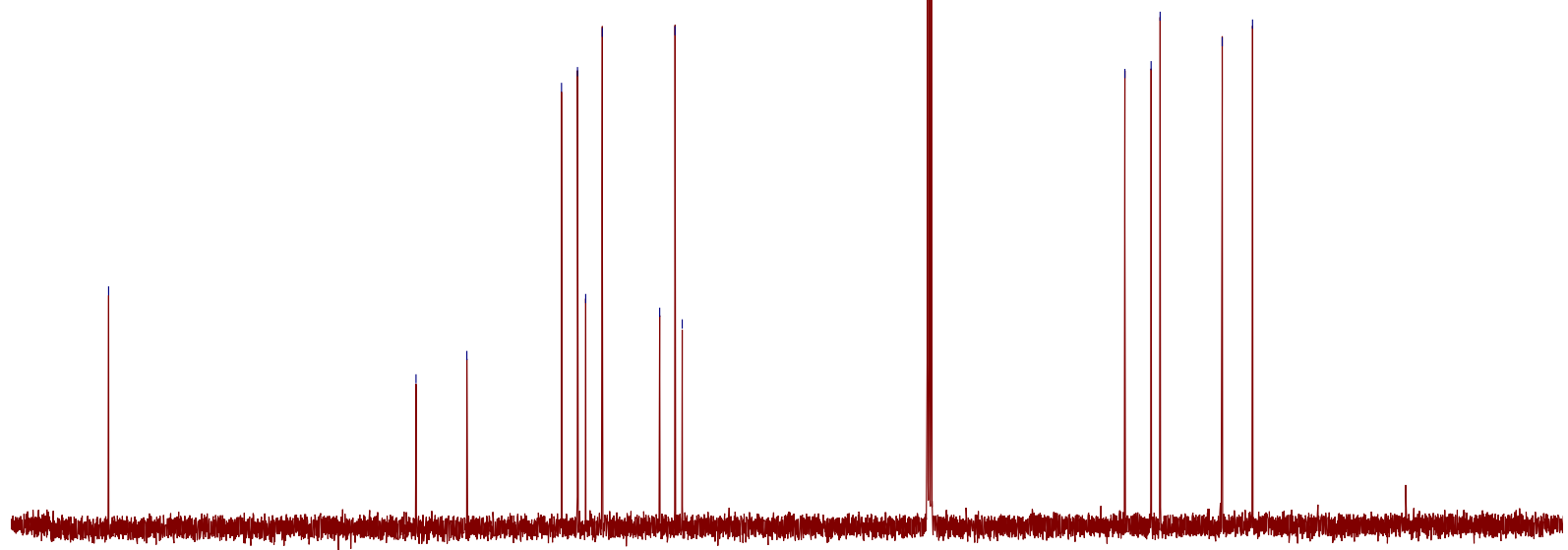

$\begin{array}{llllllllllllll}220 & 210 & 200 & 190 & 180 & 170 & 160 & 150 & 140 & 130 & 120 & 110 & 100 & 90\end{array}$
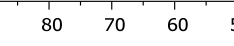

$40 \quad 30$

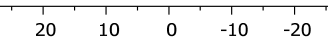




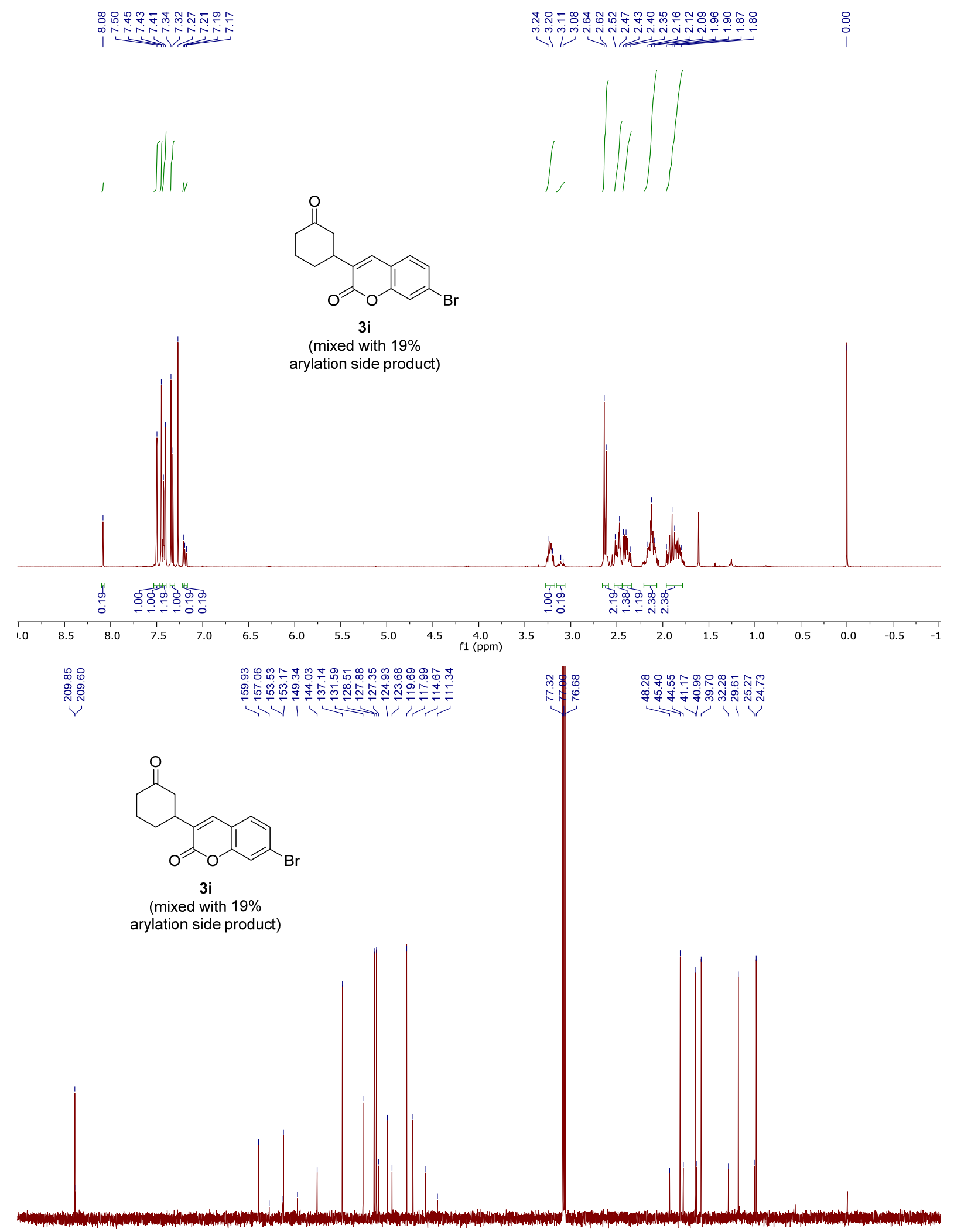

$\begin{array}{llllllllllllllllllllllllllllllllllllllll}220 & 210 & 200 & 190 & 180 & 170 & 160 & 150 & 140 & 130 & 120 & 110 & 100 & 90 & 80 & 70 & 60 & 50 & 40 & 30 & 20 & 10 & 0 & -10 & -20\end{array}$ 


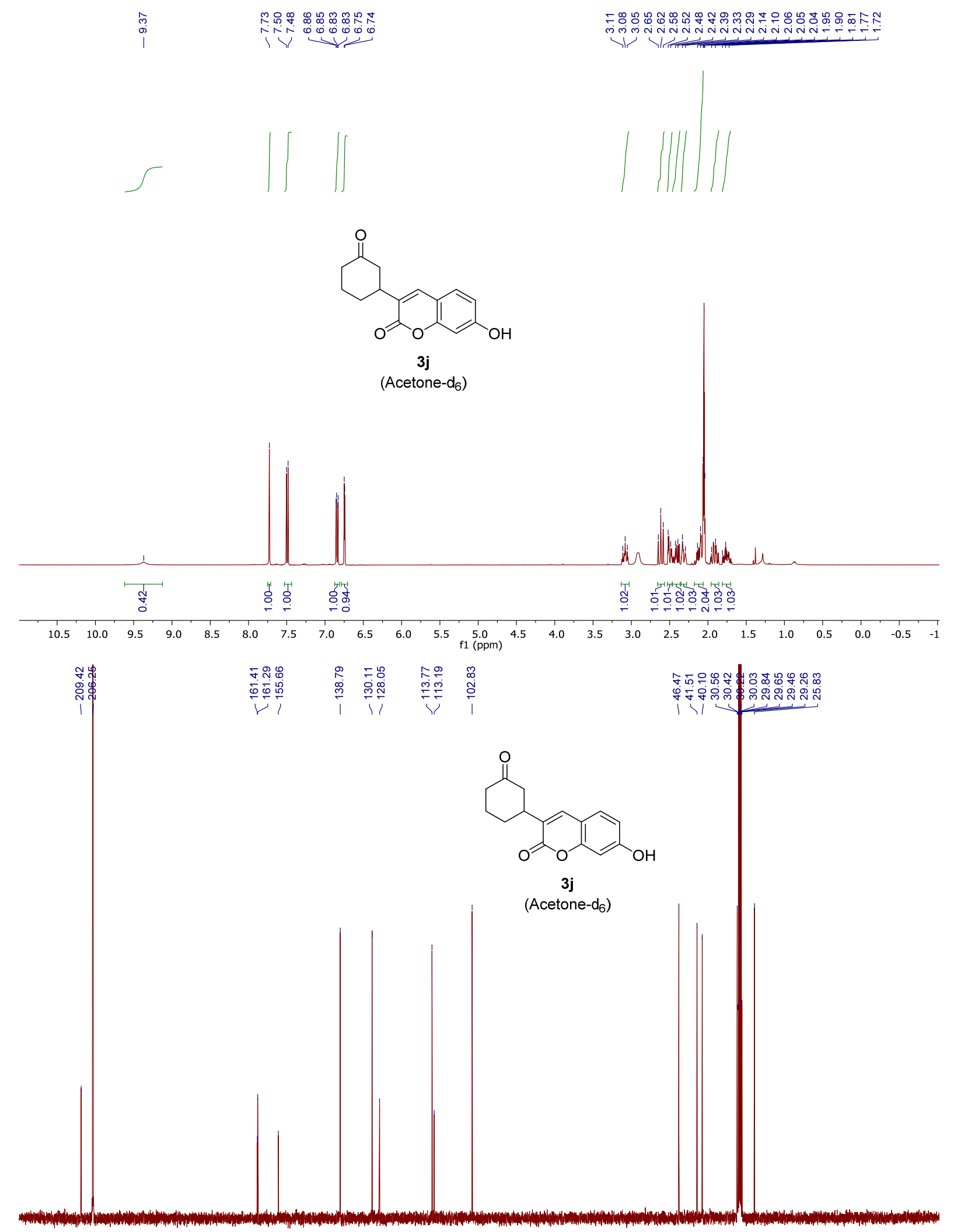

$\begin{array}{lllllllllllllllllllllllllllllll} & 220 & 210 & 200 & 190 & 180 & 170 & 160 & 150 & 140 & 130 & 120 & 110 & 100 & 90 & 80 & 70 & 60 & 50 & 40 & 30 & 20 & 10 & 0 & -10 & -20\end{array}$ 


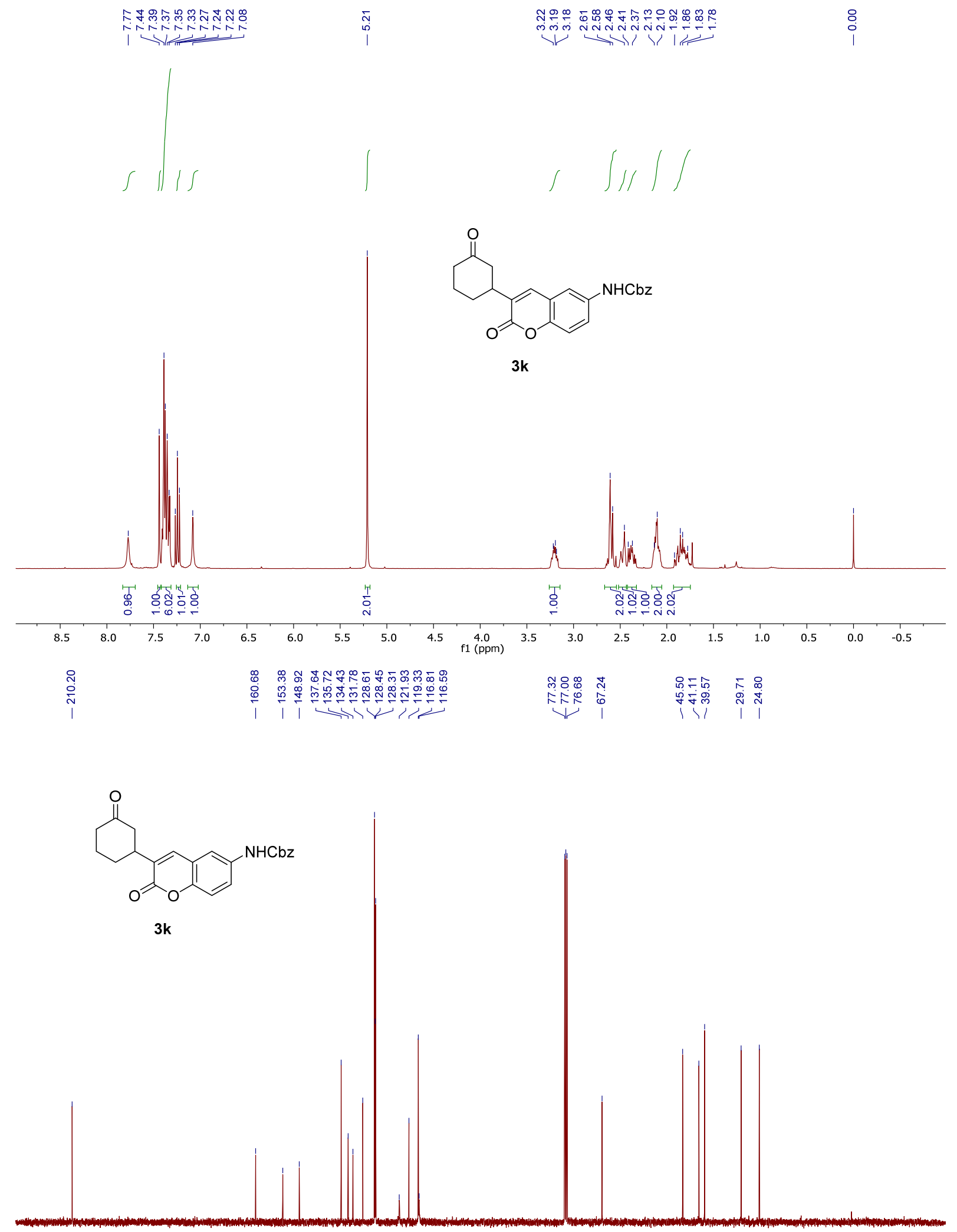

$\begin{array}{llllllllllllllllllllllllllllllll}220 & 210 & 200 & 190 & 180 & 170 & 160 & 150 & 140 & 130 & 120 & 110 & 100 & 90 & 80 & 70 & 60 & 50 & 40 & 30 & 20 & 10 & 0 & -10 & -20\end{array}$ 


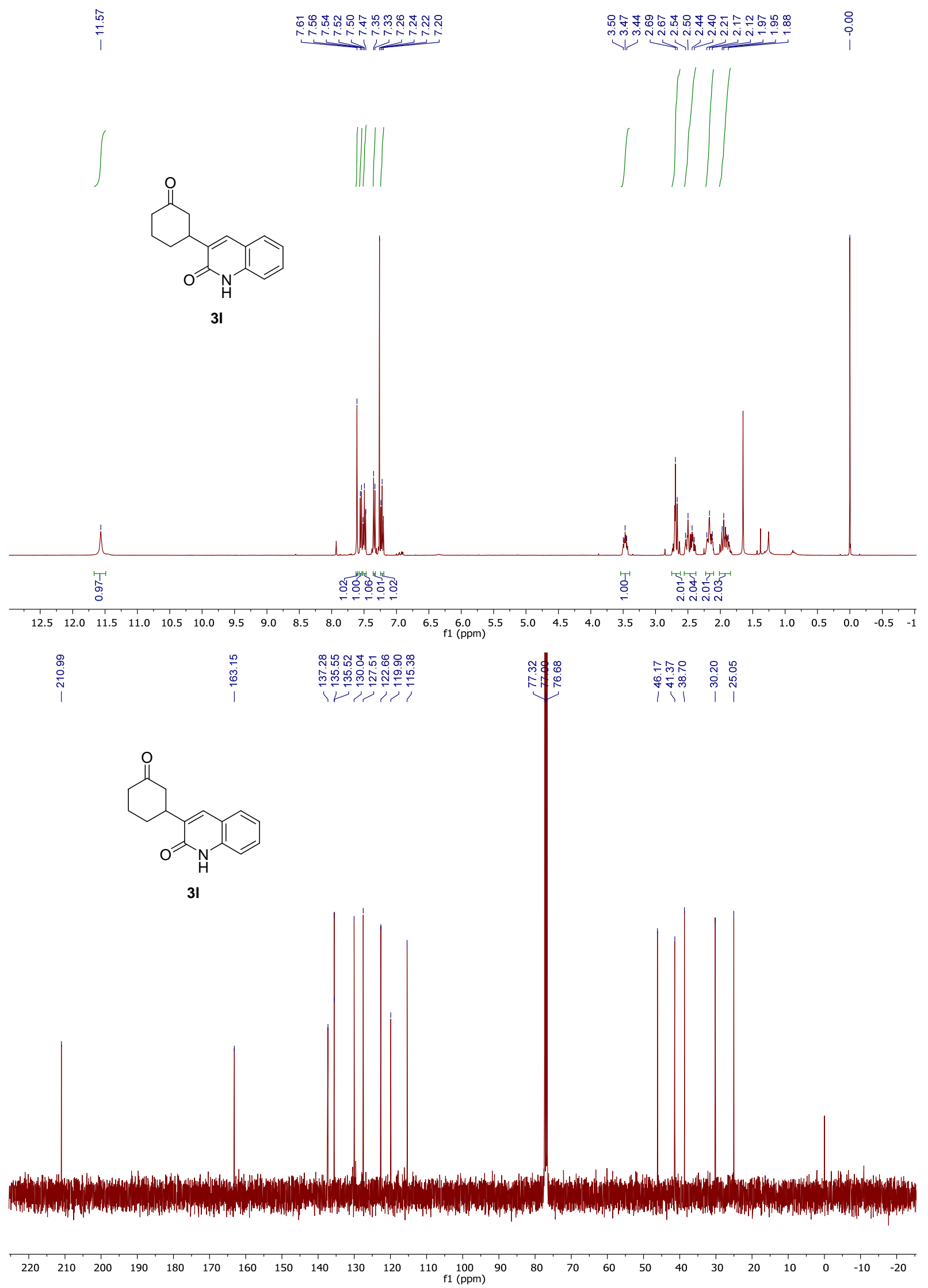




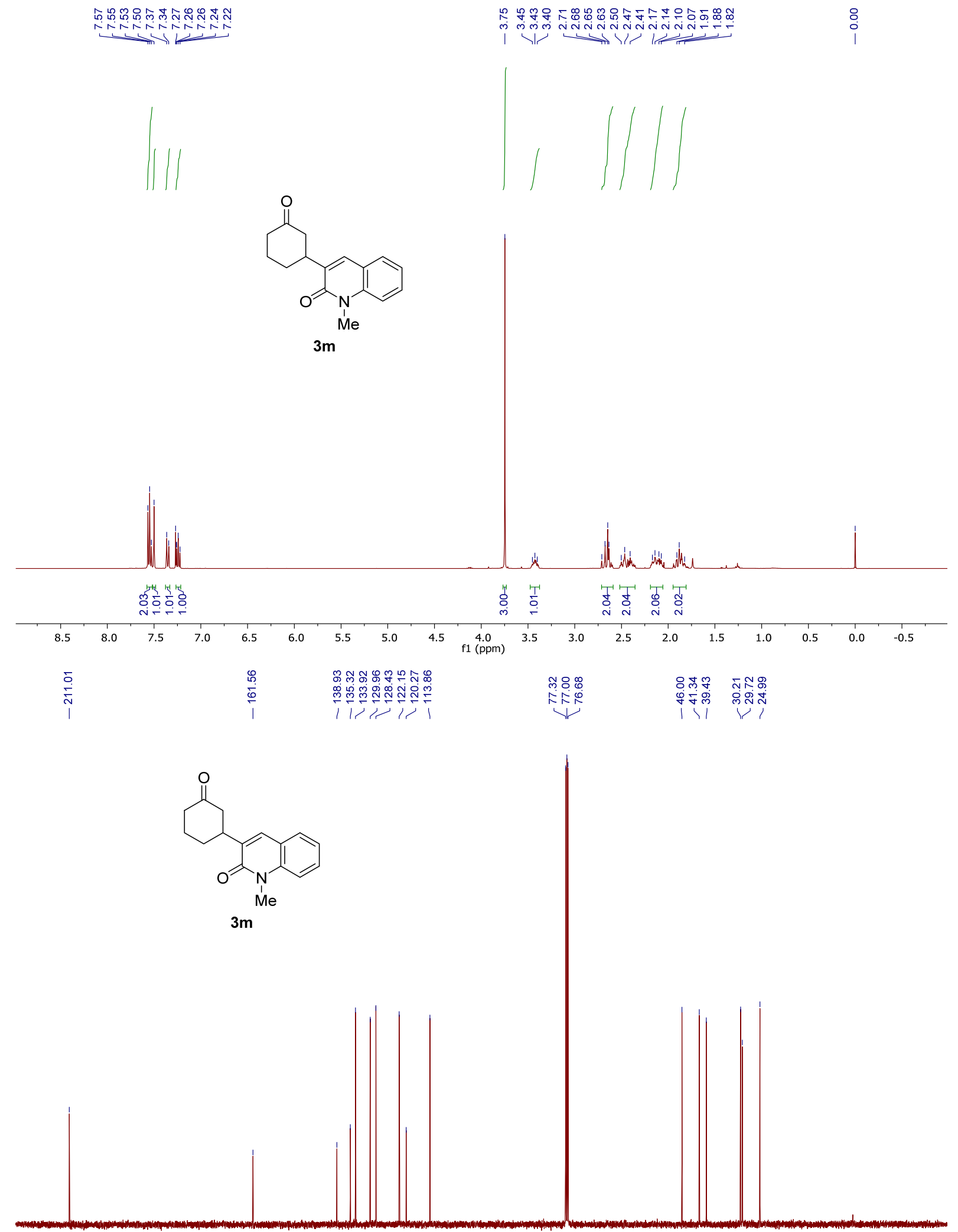



m

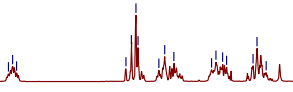

党 胥 

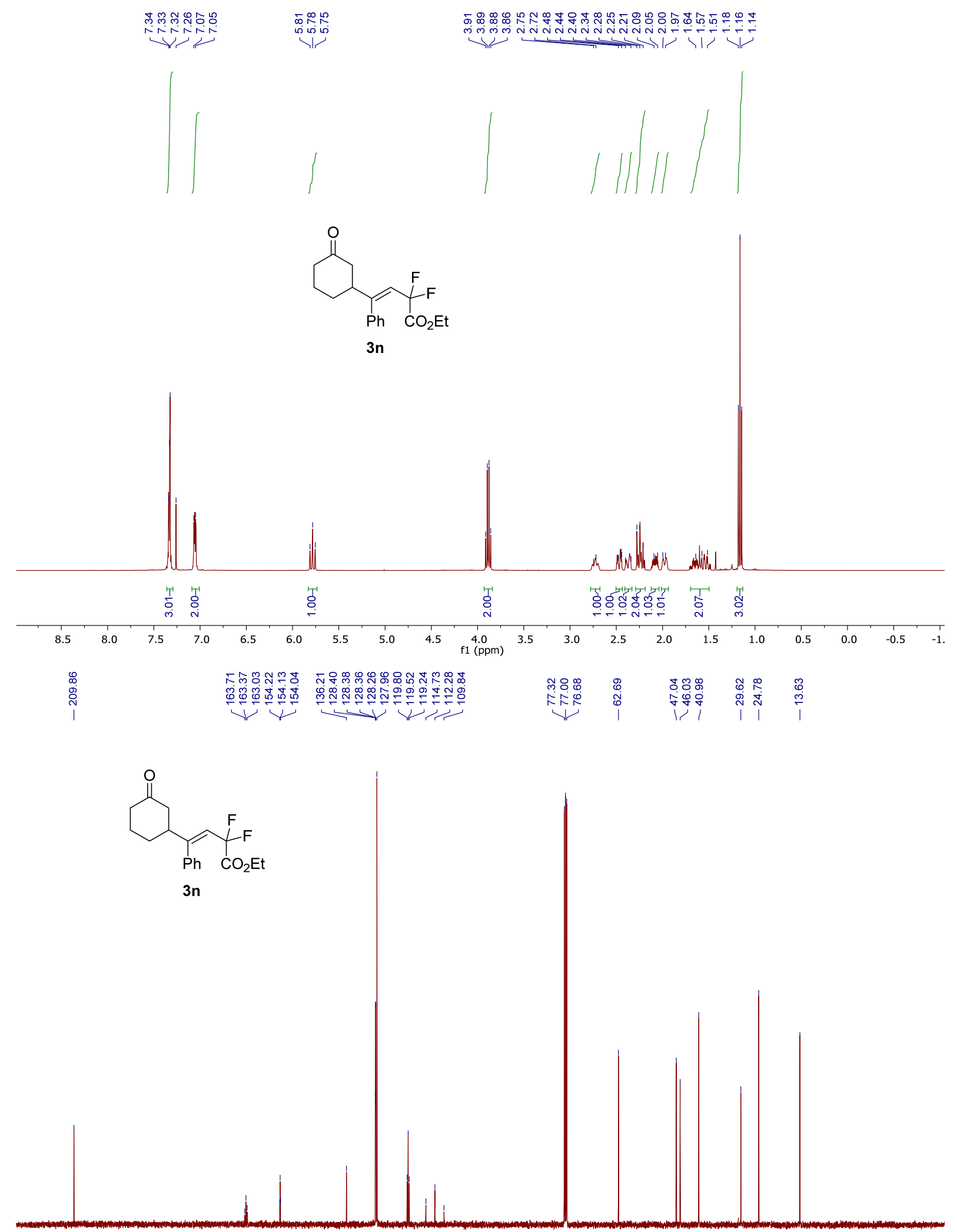

$\begin{array}{lllllllllllllllllllllllllllllllllllllllll}220 & 210 & 200 & 190 & 180 & 170 & 160 & 150 & 140 & 130 & 120 & 110 & 100 & 90 & 80 & 70 & 60 & 50 & 40 & 30 & 20 & 10 & 0 & -10 & -20\end{array}$ 


\section{${ }^{19}$ F NMR of 3n}
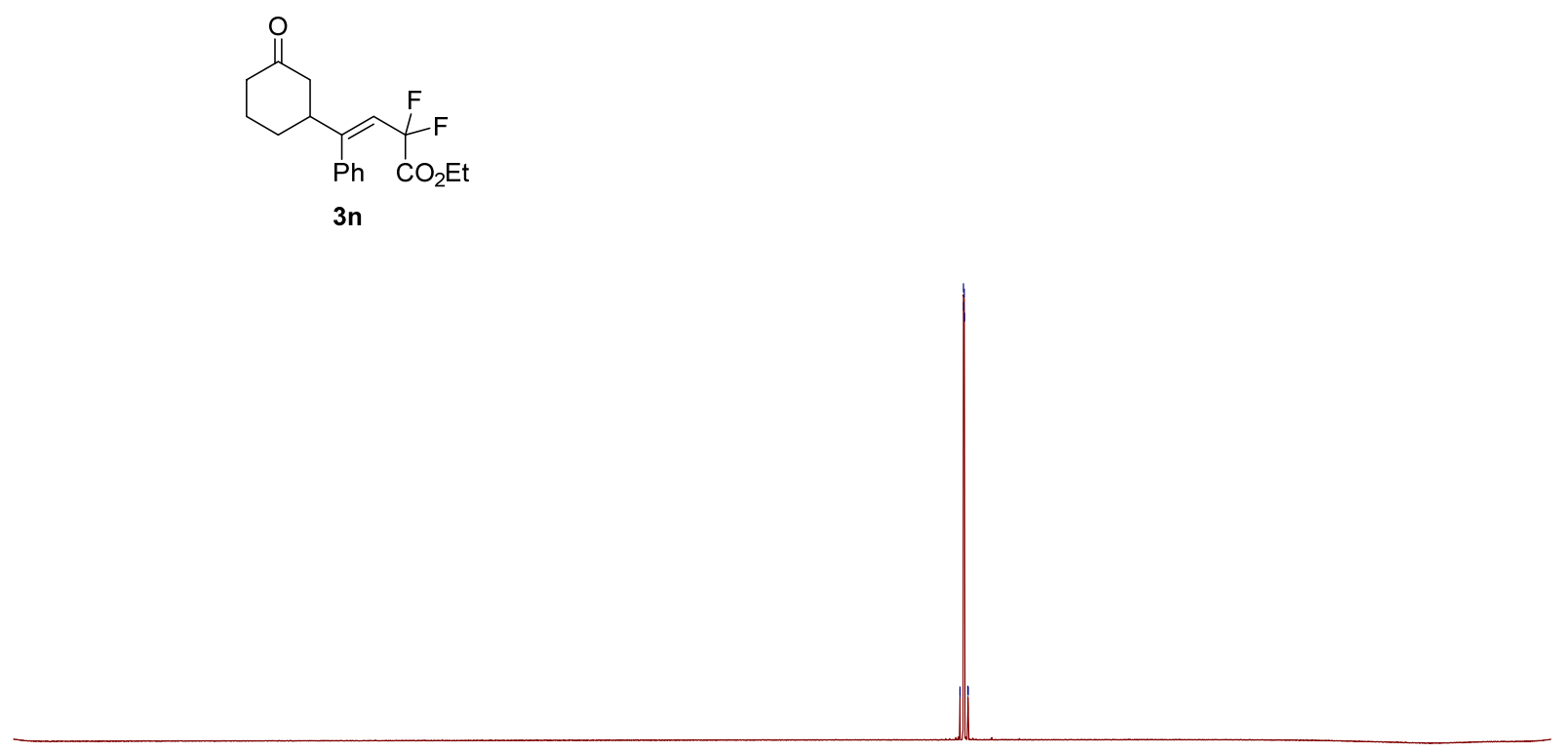

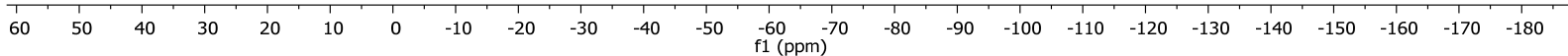




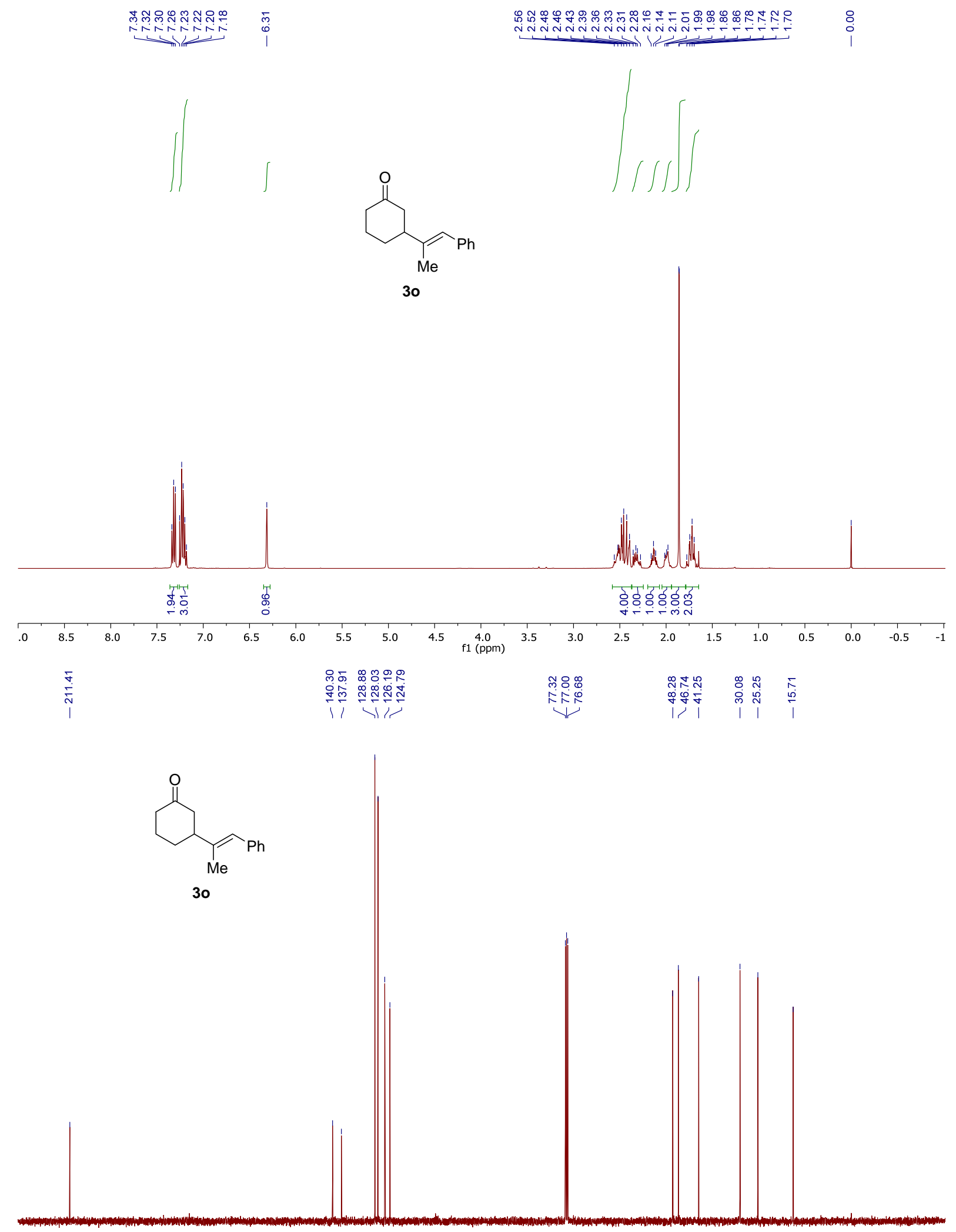

(2)

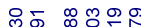

至

กิ

戚量

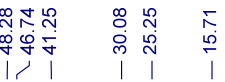

$\begin{array}{llllllllllllll}220 & 210 & 200 & 190 & 180 & 170 & 160 & 150 & 140 & 130 & 120 & 110 & 100 & 90\end{array}$

80

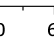

50

40

$\begin{array}{lllll}20 & 10 & 0 & -10 & -20\end{array}$ 


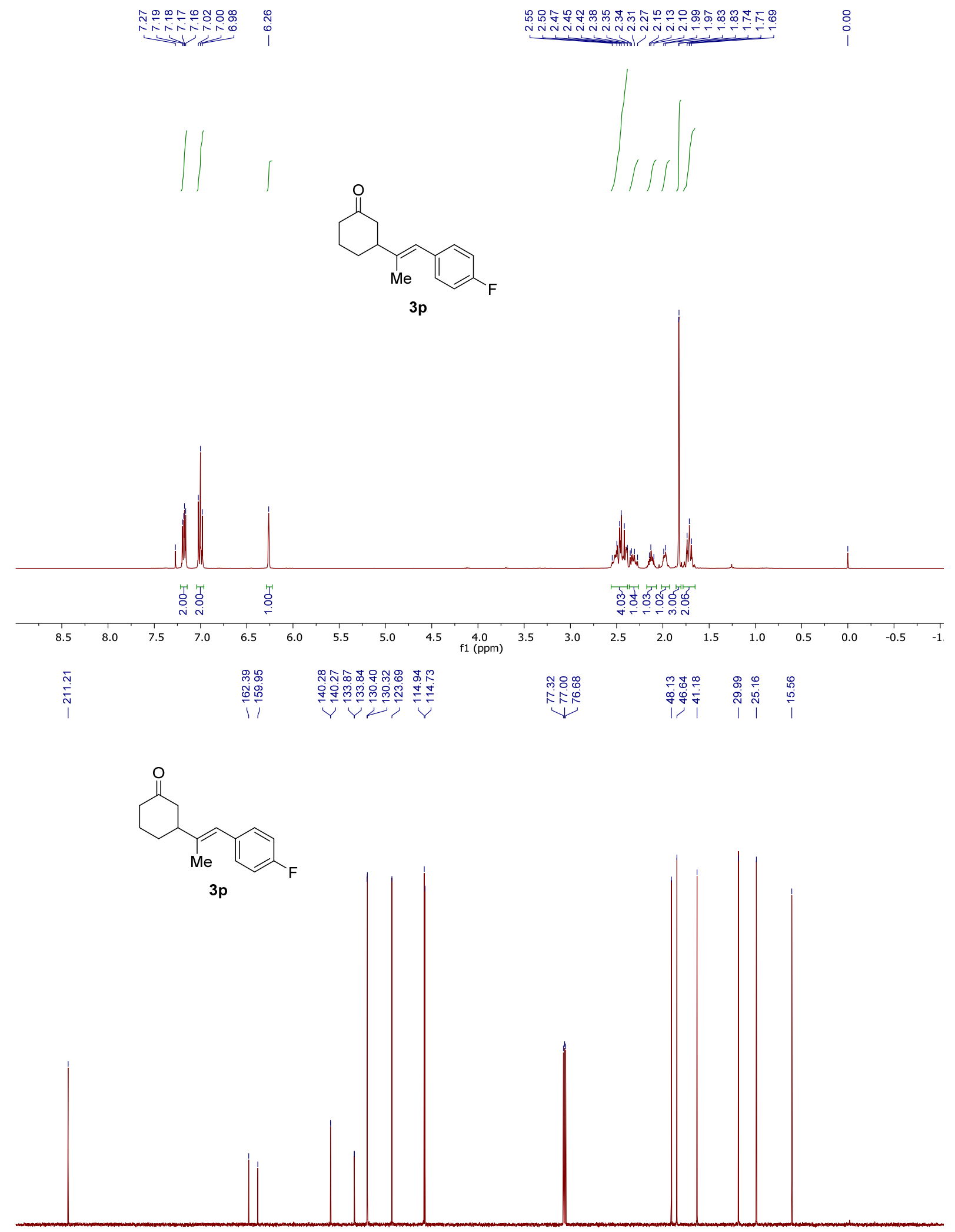

$\begin{array}{lllllllllllllllllllllllllllll} & 1 \\ 220 & 210 & 200 & 190 & 180 & 170 & 160 & 150 & 140 & 130 & 120 & 110 & 100 & 90 & 80 & 70 & 60 & 50 & 40 & 30 & 20 & 10 & 0 & -10 & -20\end{array}$ 


\section{${ }^{19}$ F NMR of 3p}

$\operatorname{lich}$

곤

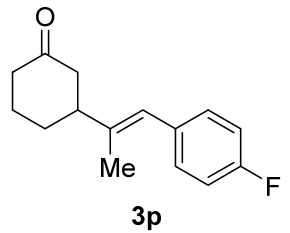

$\begin{array}{llllllllllllllllllllllllll}60 & 50 & 40 & 30 & 20 & 10 & 0 & -10 & -20 & -30 & -40 & -50 & -60 & -70 & -80 & -90 & -100 & -110 & -120 & -130 & -140 & -150 & -160 & -170 & -180\end{array}$ 


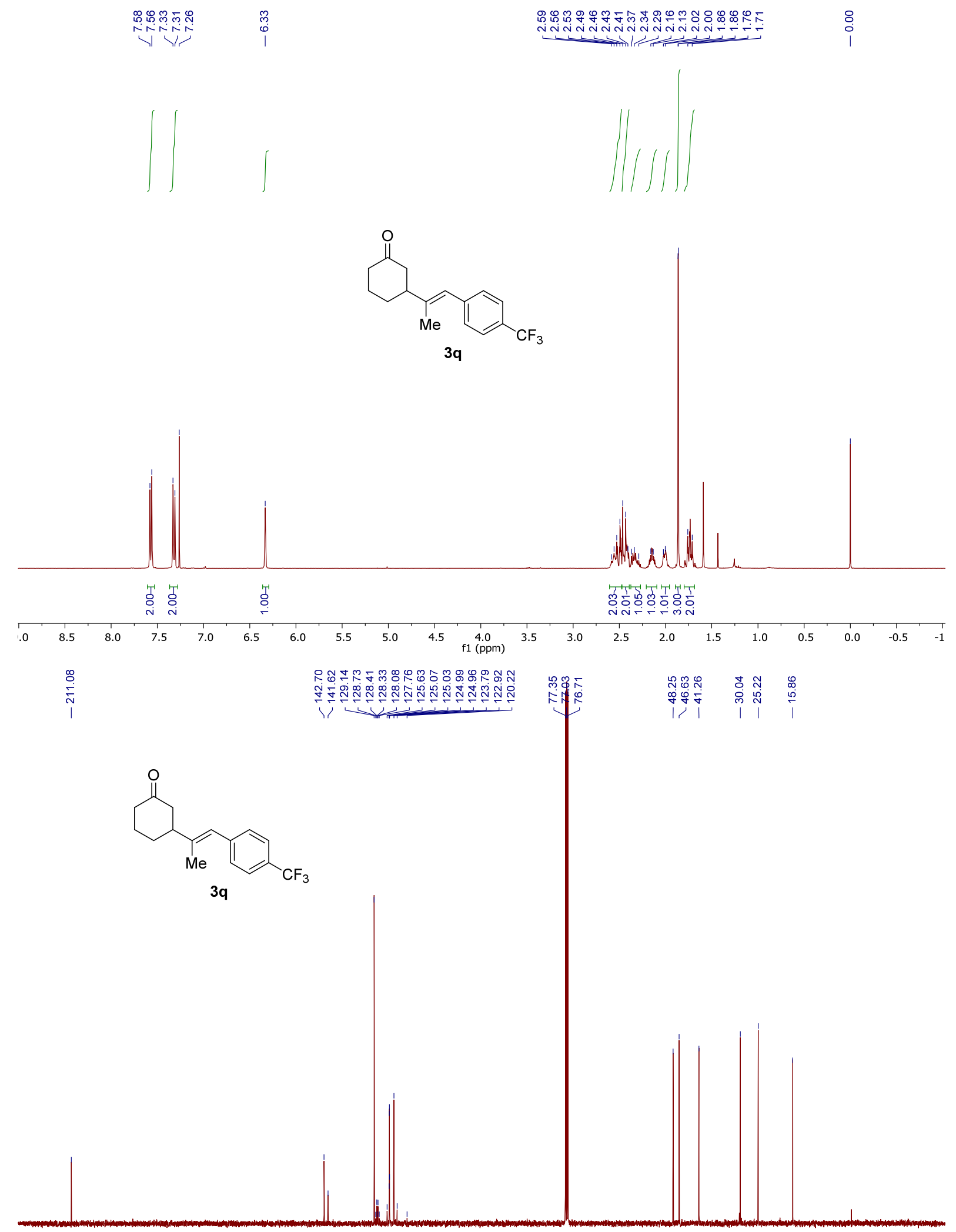

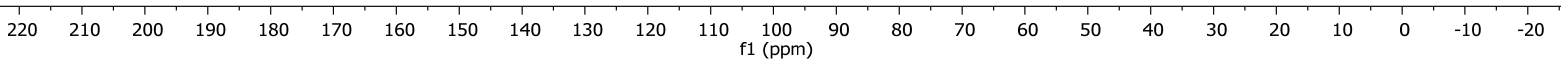


${ }^{19}$ F NMR of 3q

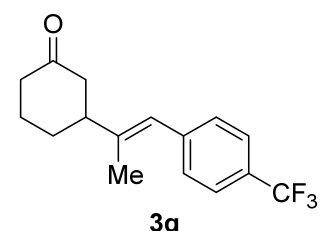

\begin{tabular}{lllllllllllllllllllllllllll}
\hline 60 & 50 & 40 & 30 & 20 & 10 & 0 & -10 & -20 & -30 & -40 & -50 & -60 & -70 & -80 & -90 & -100 & -110 & -120 & -130 & -140 & -150 & -160 & -170 & -180
\end{tabular} 

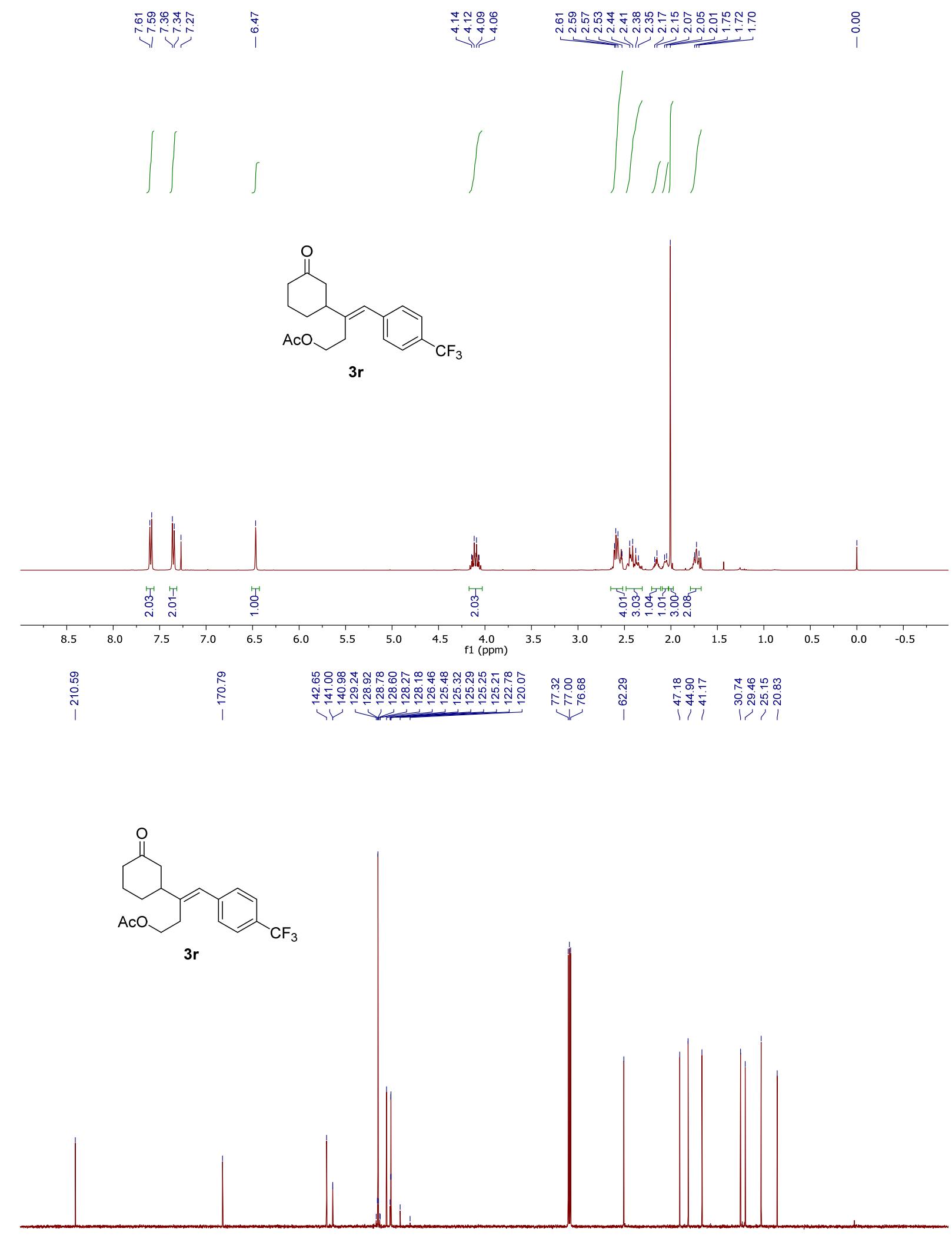

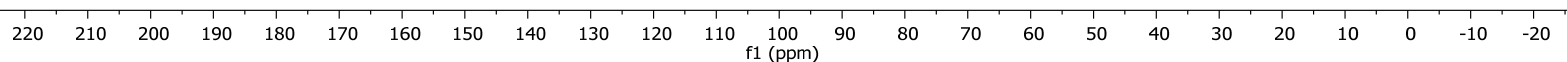




\section{${ }^{19}$ F NMR of 3r}

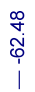

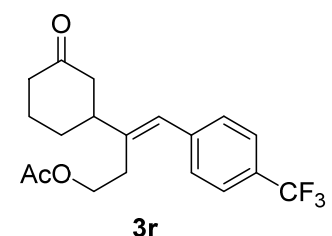

$3 r$

$\begin{array}{lllllllllllllllllllllllllllll} & 60 & 50 & 40 & 30 & 20 & 10 & 0 & -10 & -20 & -30 & -40 & -50 & -60 & -70 & -80 & -90 & -100 & -110 & -120 & -130 & -140 & -150 & -160 & -170 & -180\end{array}$ 


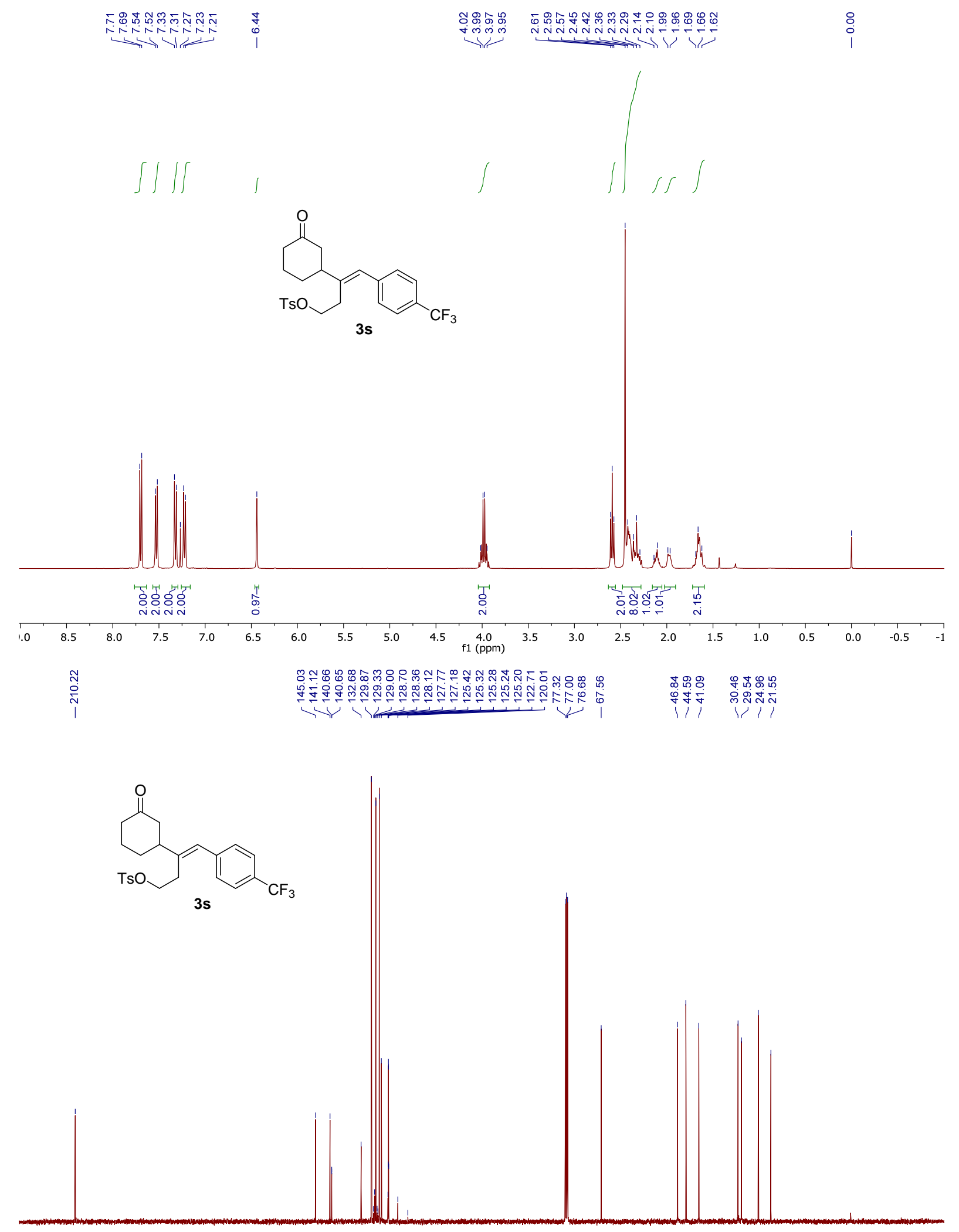

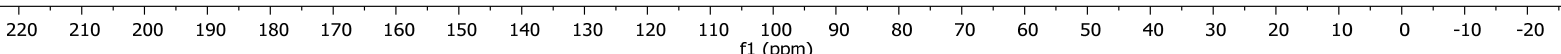




\section{${ }^{19}$ F NMR of $3 \mathrm{~s}$}

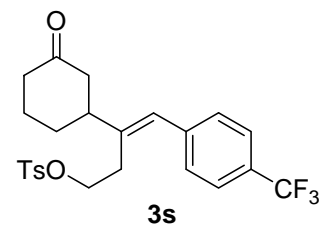

$\begin{array}{llllllllllllllllllllllllll}60 & 50 & 40 & 30 & 20 & 10 & 0 & -10 & -20 & -30 & -40 & -50 & -60 & -70 & -80 & -90 & -100 & -110 & -120 & -130 & -140 & -150 & -160 & -170 & -180\end{array}$ 


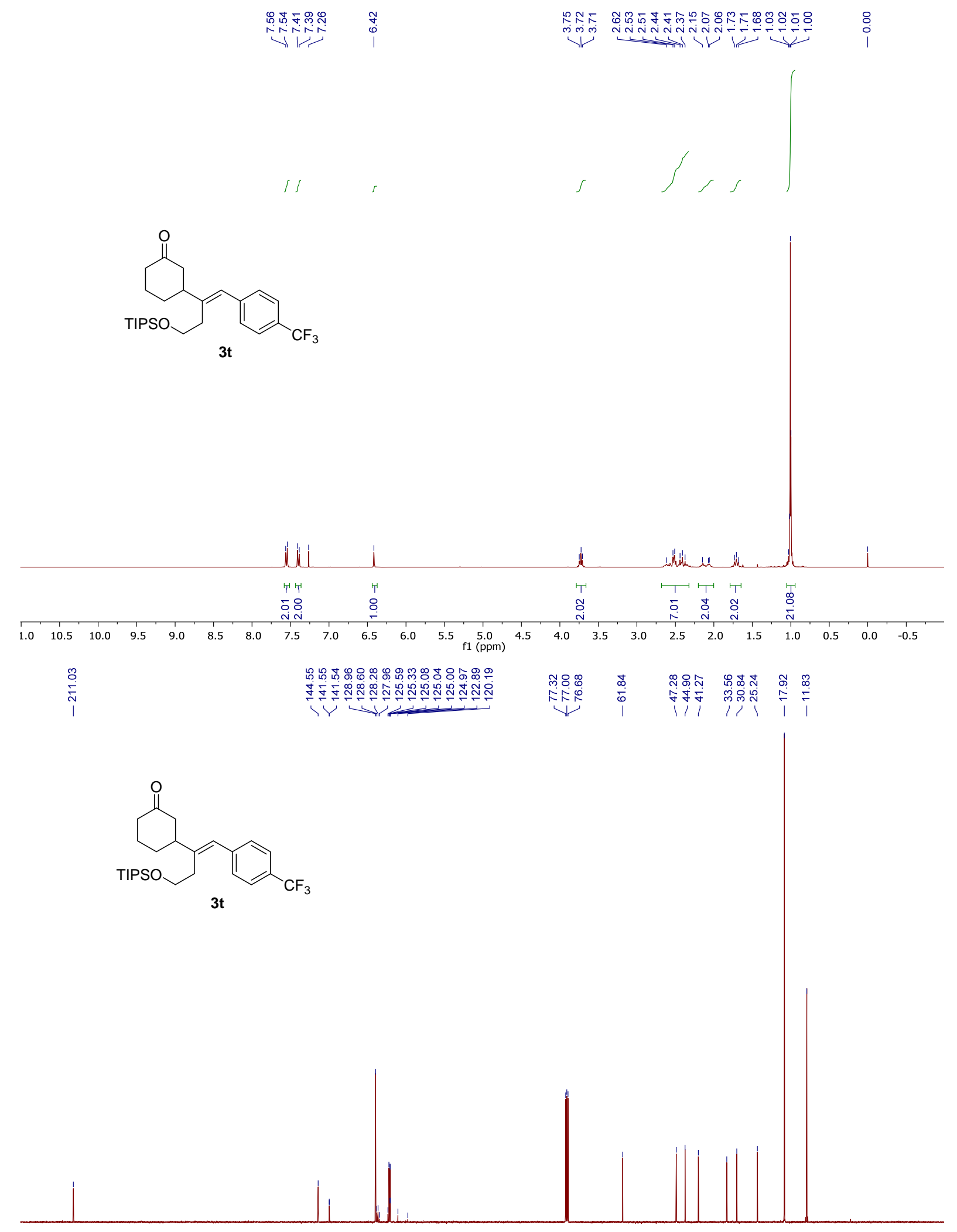

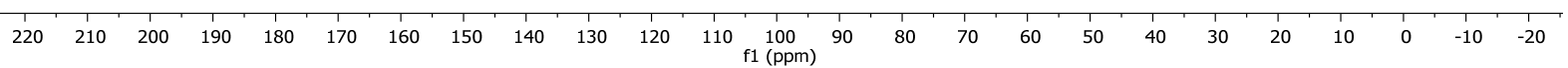




\section{${ }^{19}$ F NMR of $3 t$}

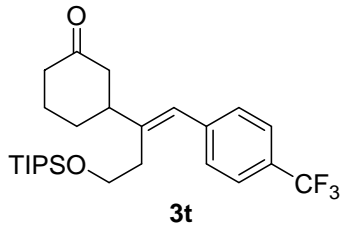



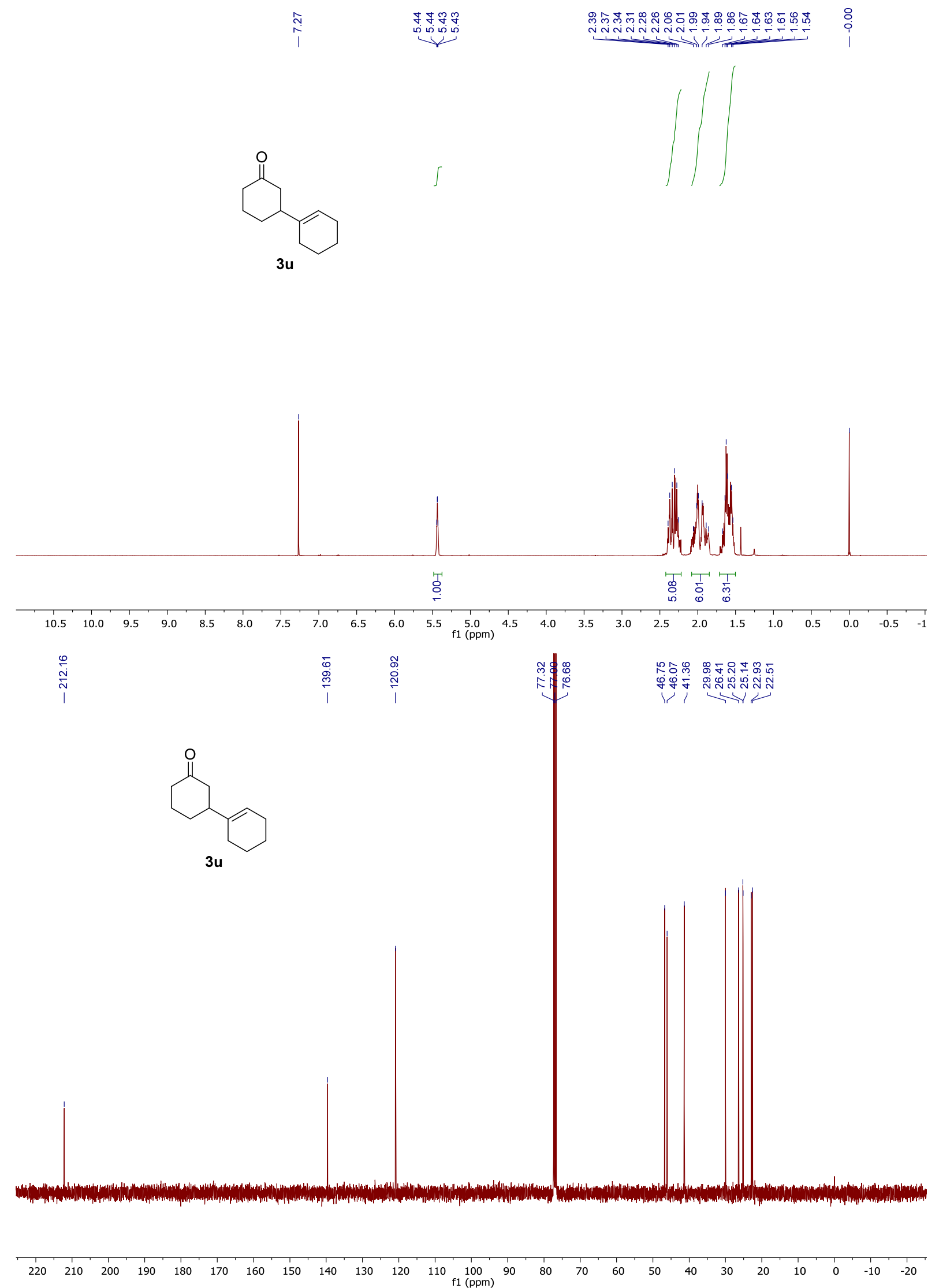


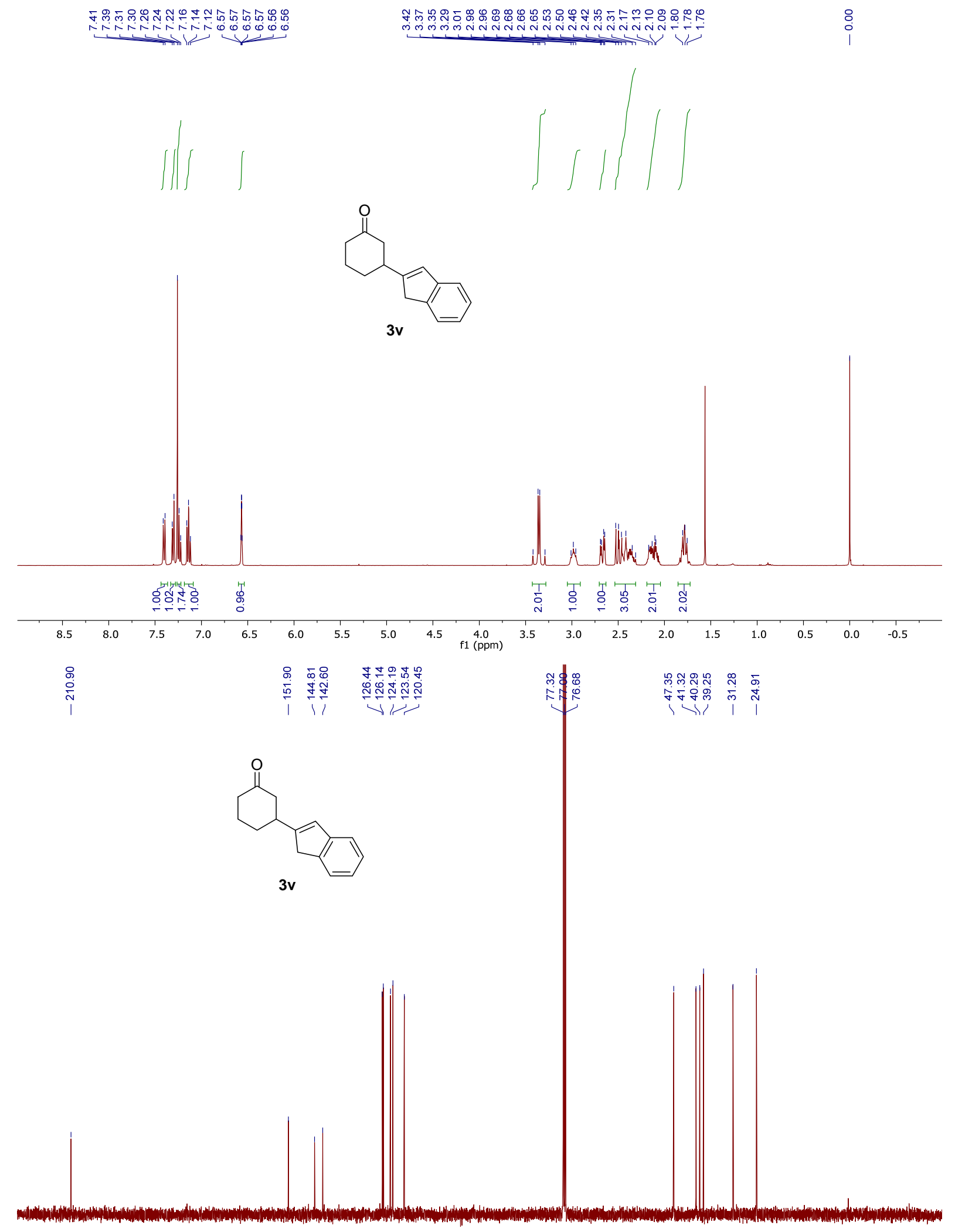

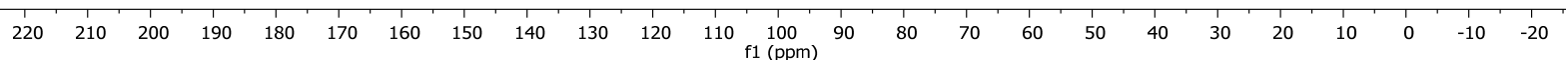



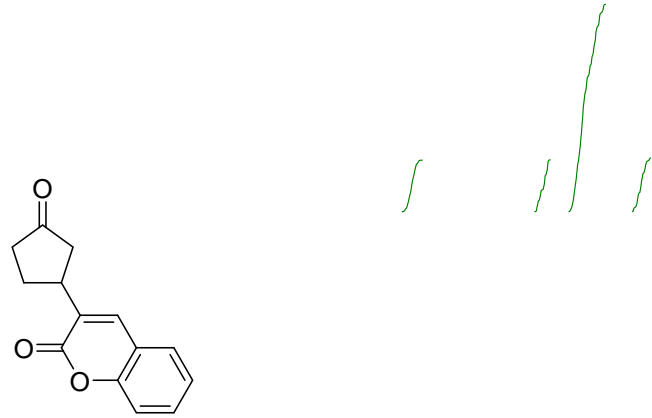

$4 a$

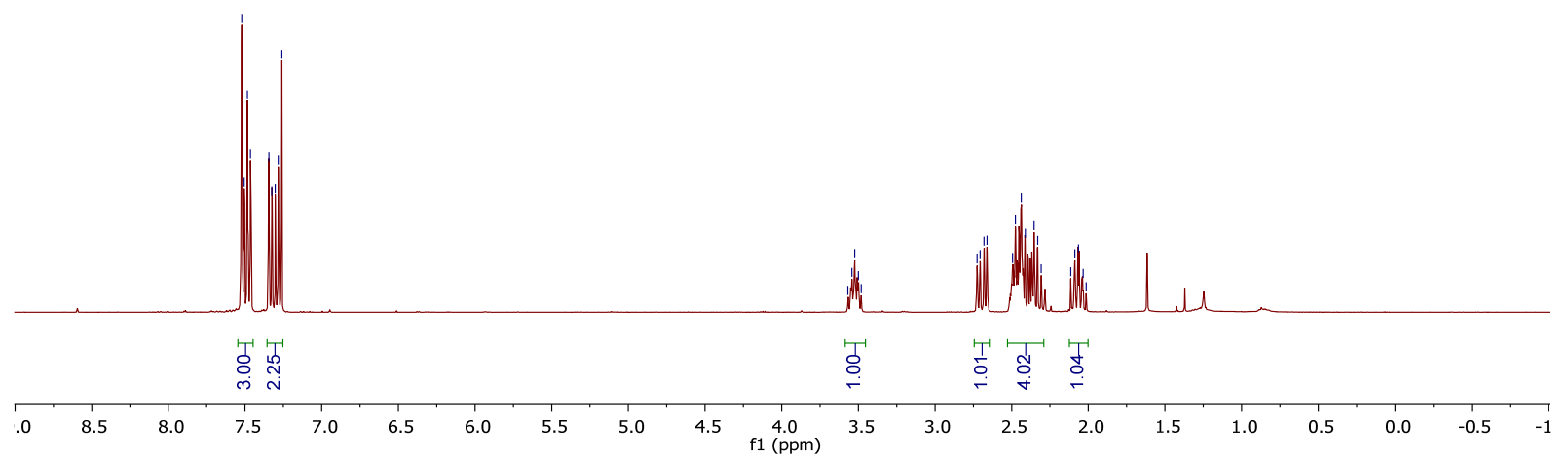

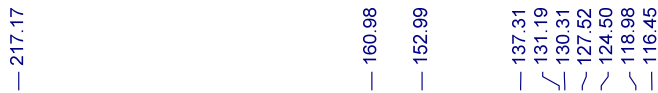

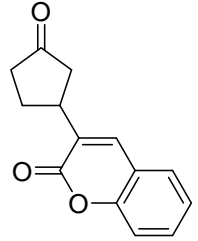

$4 a$

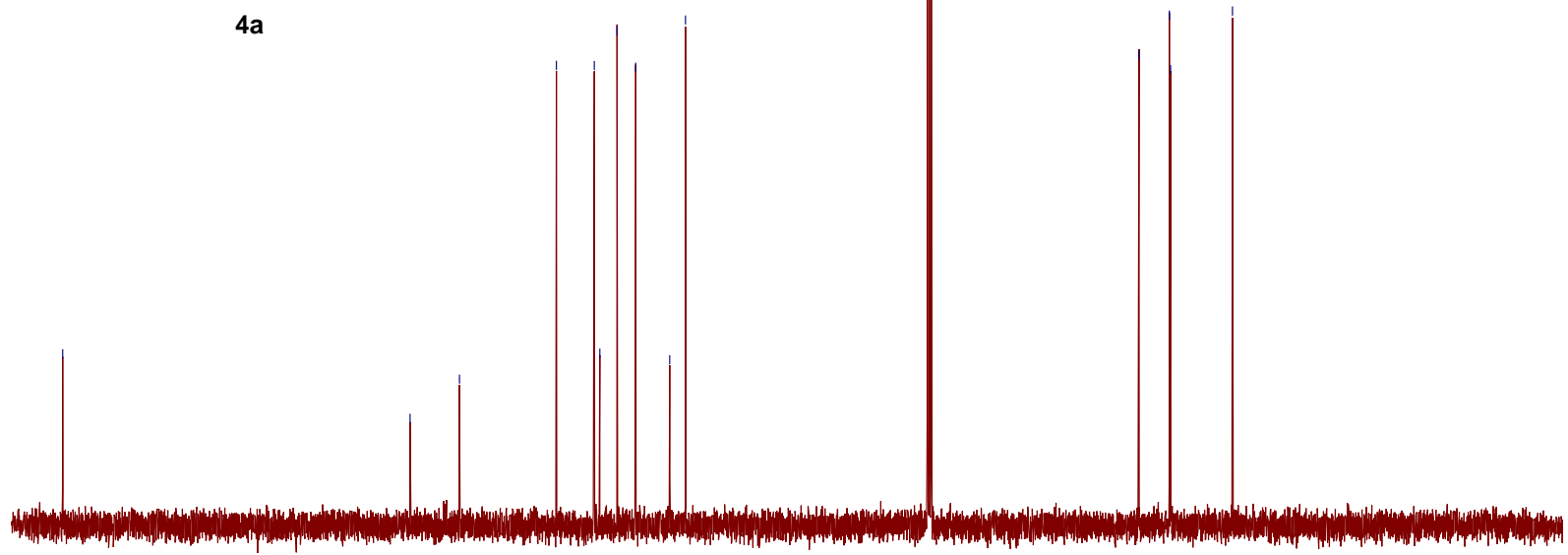

$\begin{array}{llllllllllllll}220 & 210 & 200 & 190 & 180 & 170 & 160 & 150 & 140 & 130 & 120 & 110 & 100 & 90\end{array}$

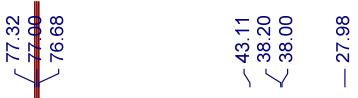




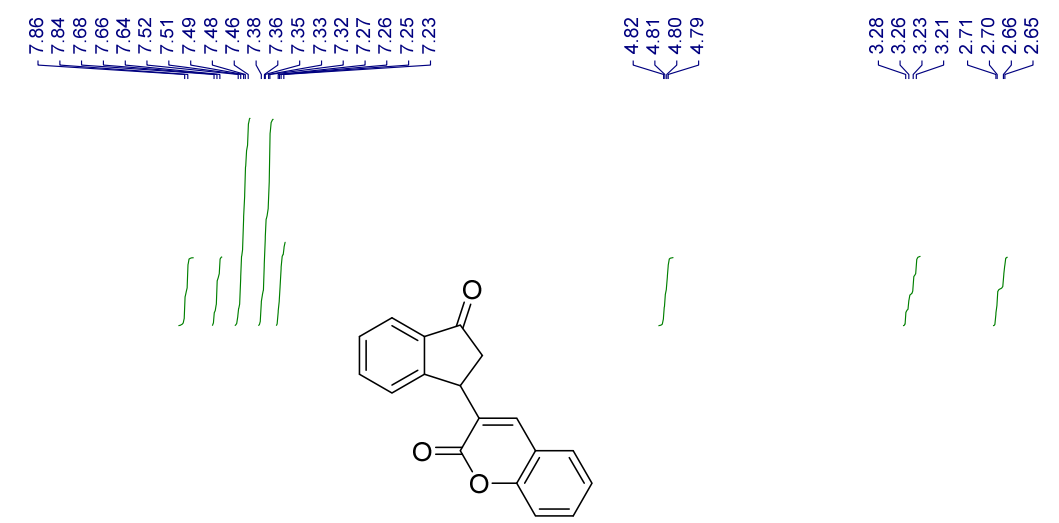

$4 \mathbf{b}$
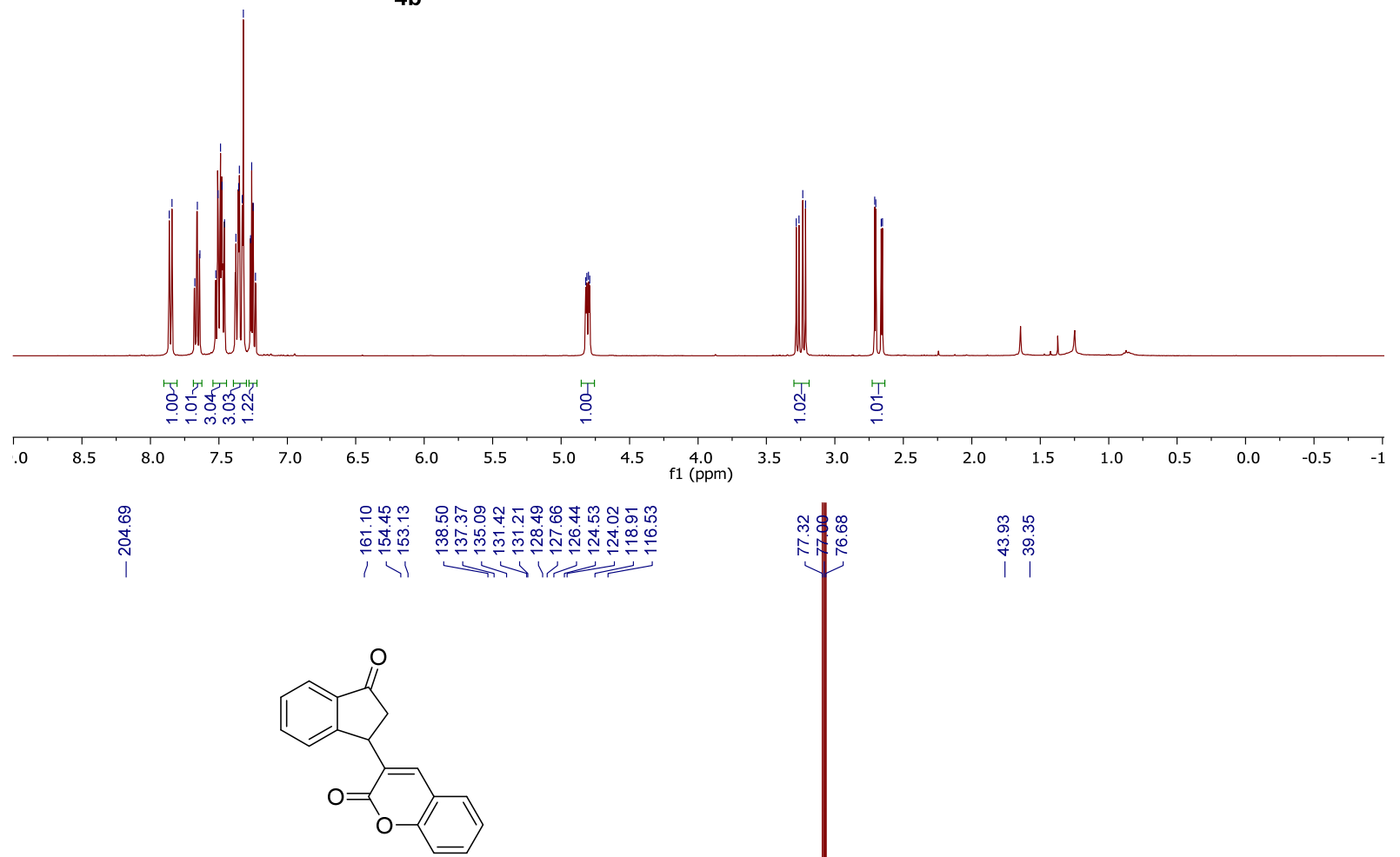

4b

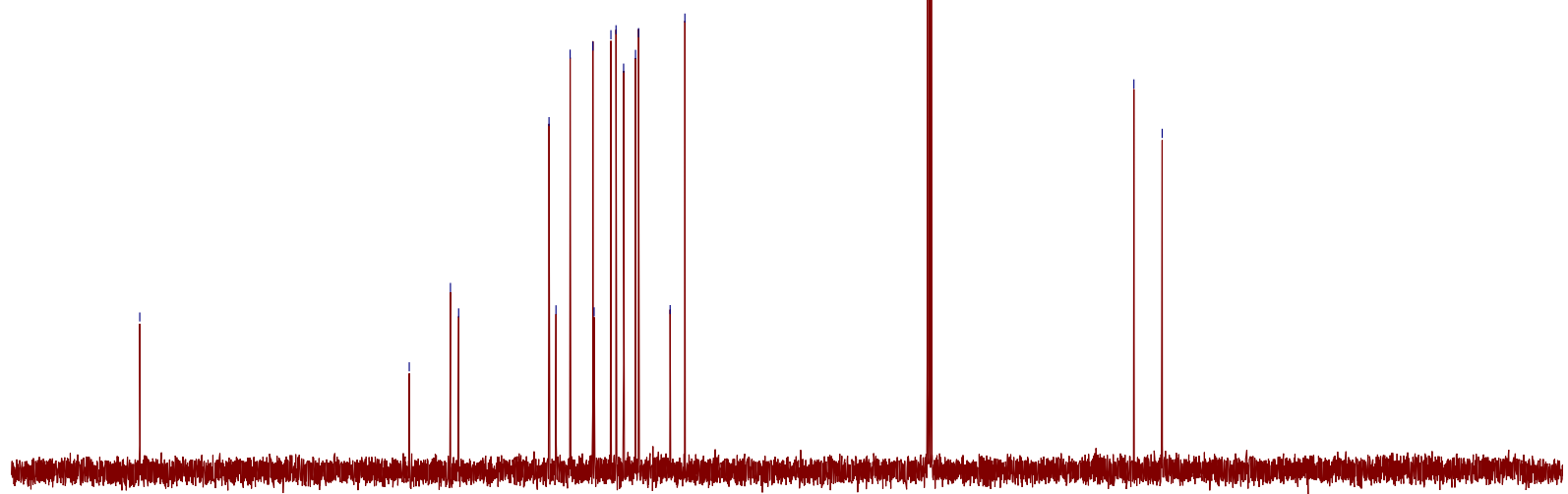

$\begin{array}{llllllllllllllllllllllllllllllllllll}220 & 210 & 200 & 190 & 180 & 170 & 160 & 150 & 140 & 130 & 120 & 110 & 100 & 90 & 80 & 70 & 60 & 50 & 40 & 30 & 20 & 10 & 0 & -10 & -20\end{array}$ 

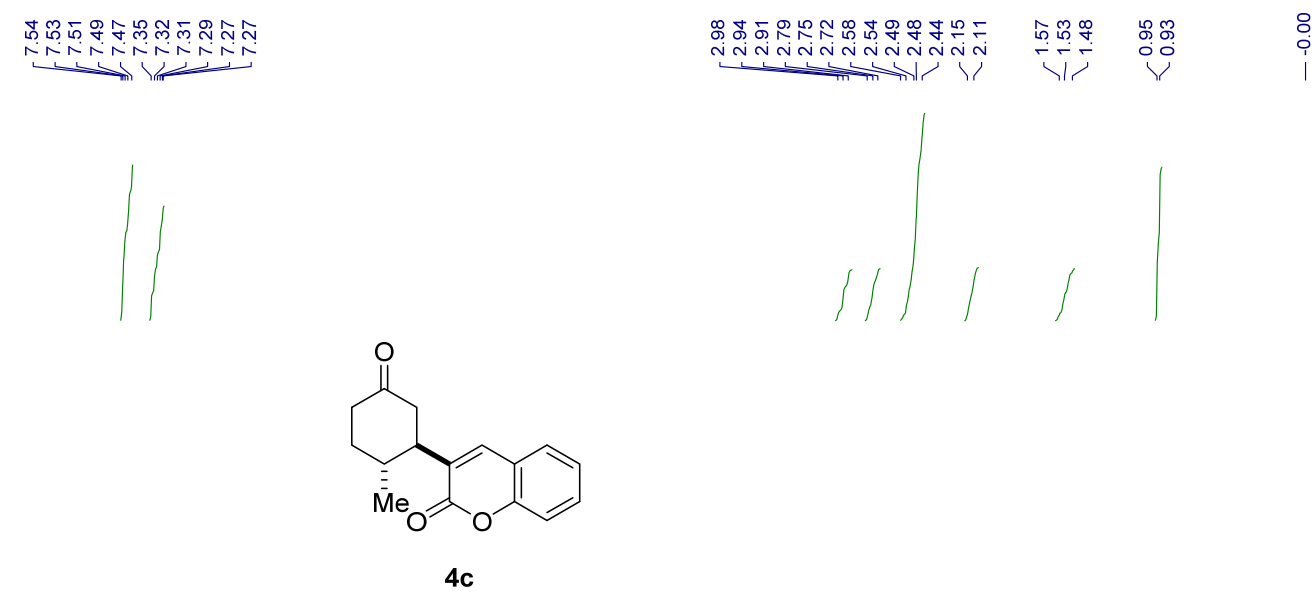

(d.r. $>20: 1)$

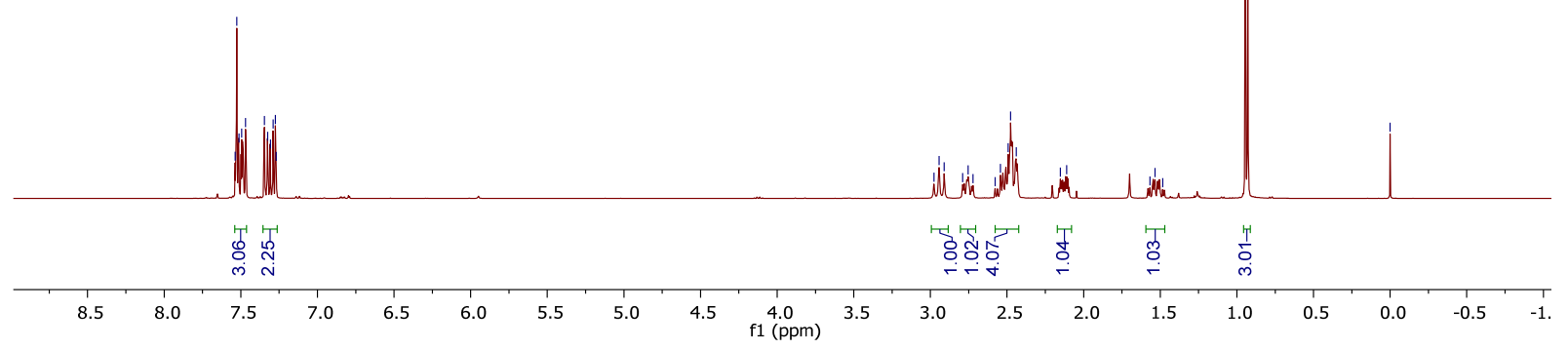

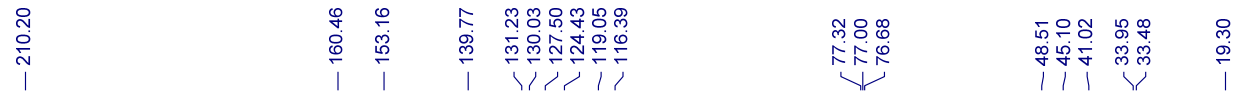

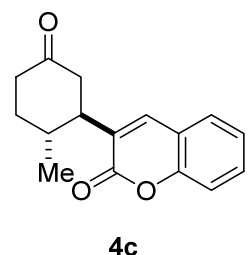

(d.r. $>20: 1)$

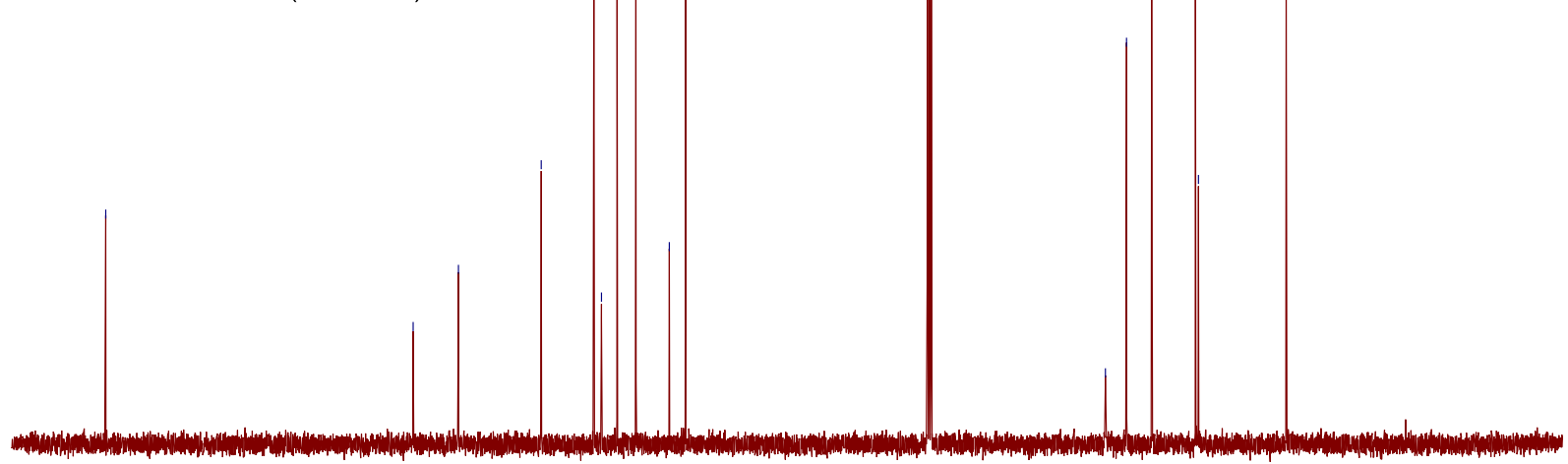

$\begin{array}{llllllllllllll}220 & 210 & 200 & 190 & 180 & 170 & 160 & 150 & 140 & 130 & 120 & 110 & 100 & 90\end{array}$

80

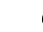

$50 \quad 40$

30

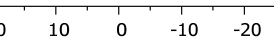




\section{COSY of 4c}
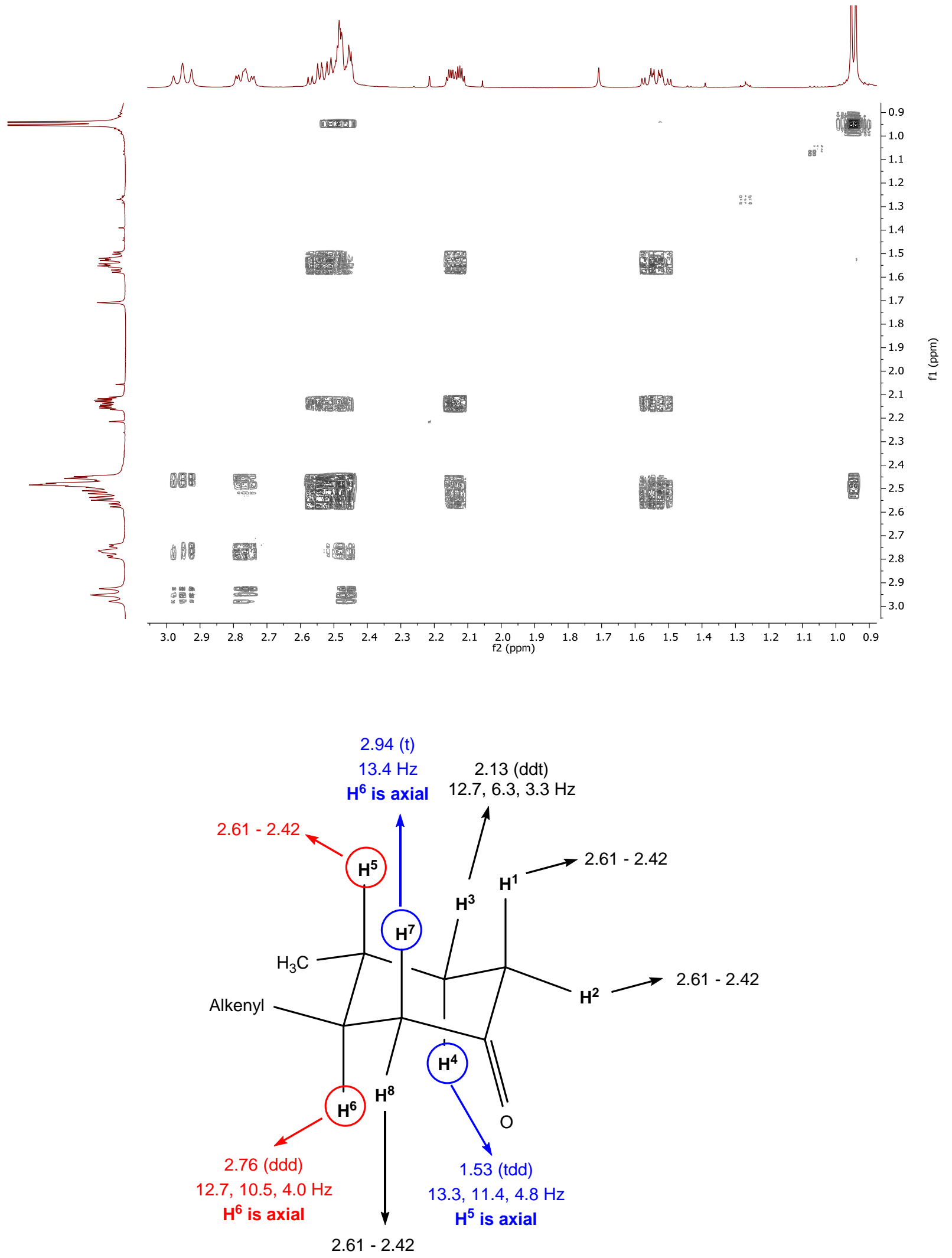

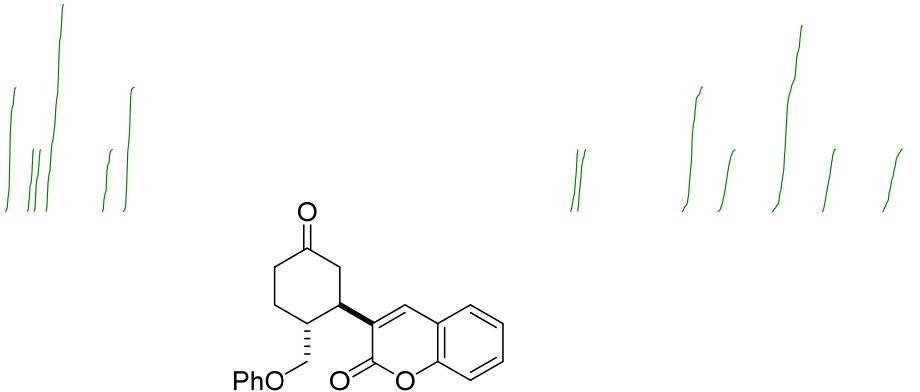

$$
\begin{gathered}
4 d \\
\text { (d.r. }>20: 1)
\end{gathered}
$$
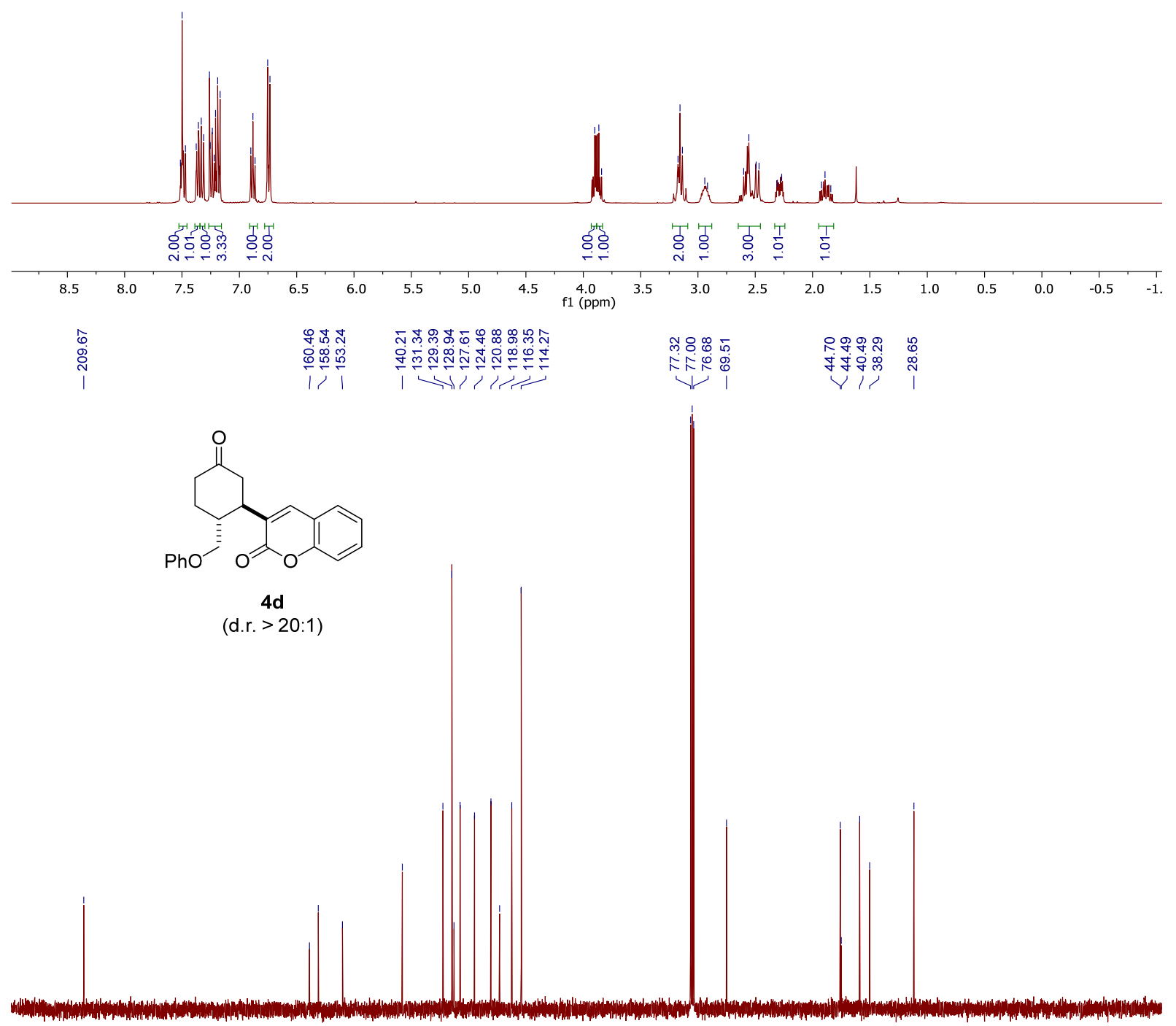

$\begin{array}{llllllllllllll}220 & 210 & 200 & 190 & 180 & 170 & 160 & 150 & 140 & 130 & 120 & 110 & 100 & 90\end{array}$
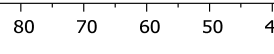

$\begin{array}{llllll}30 & 20 & 10 & 0 & -10 & -20\end{array}$ 

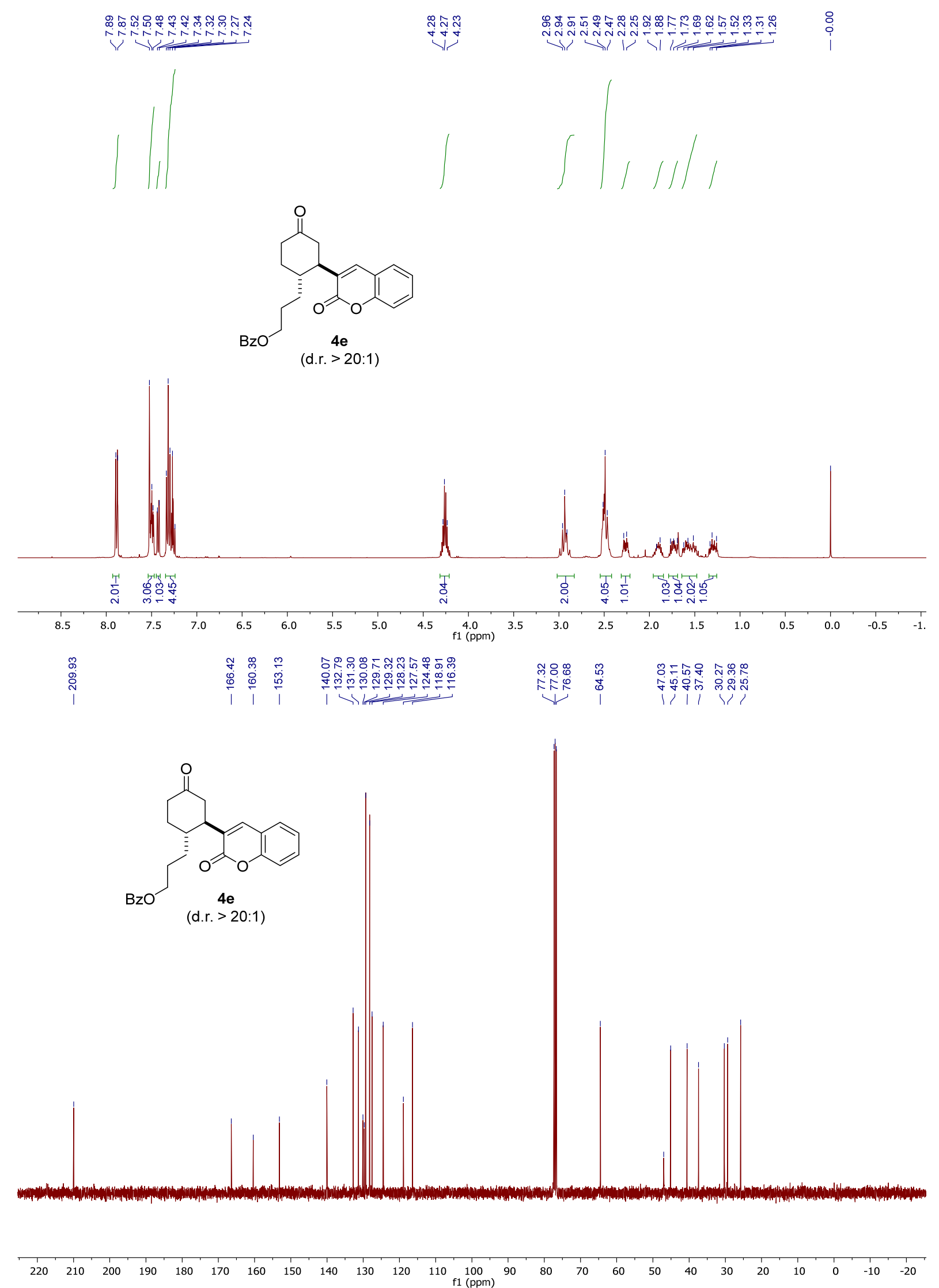


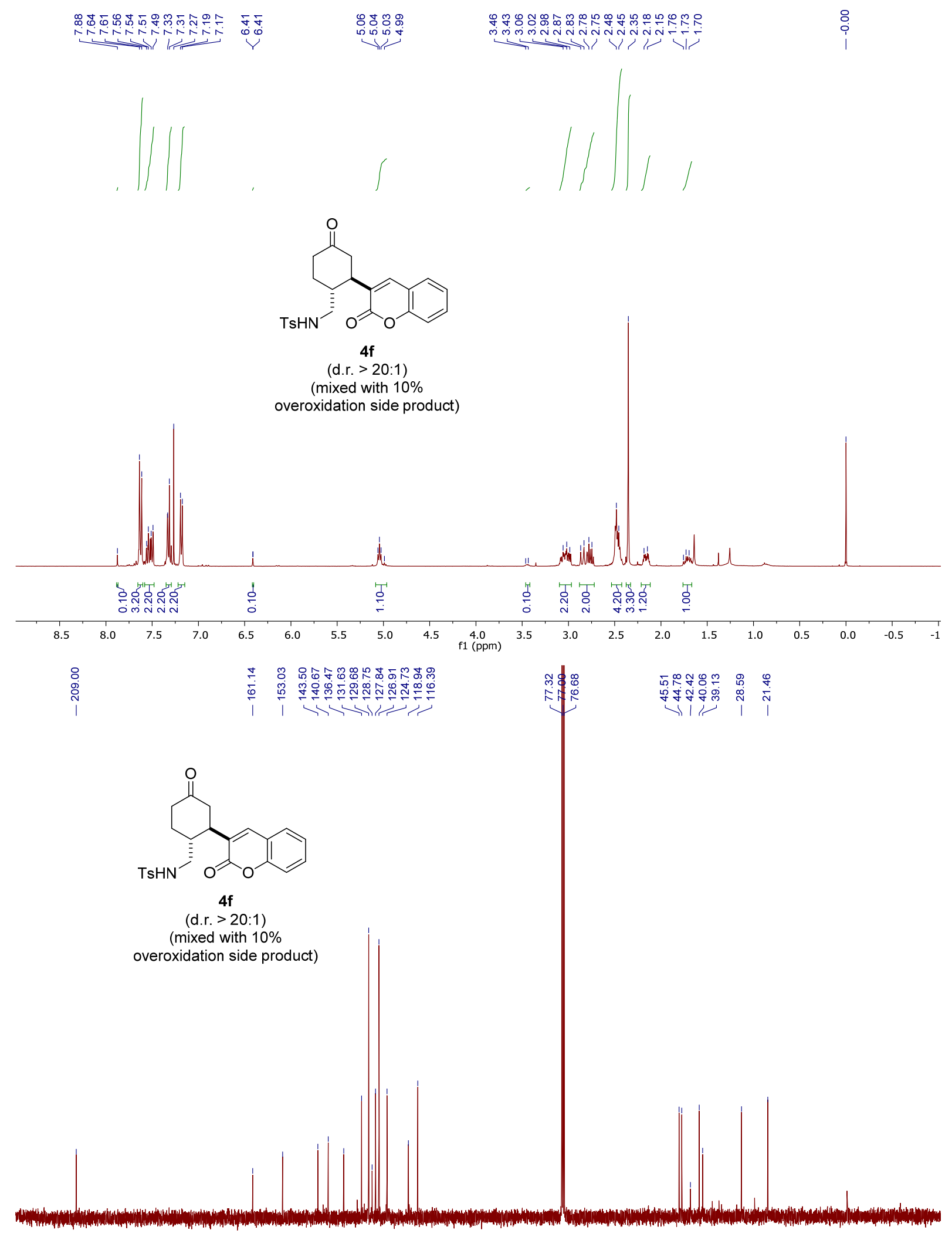

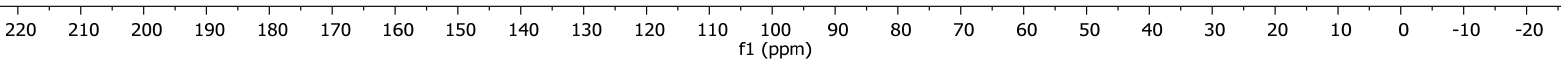




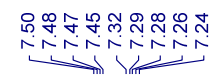

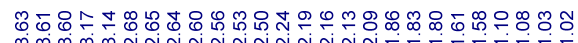

$\Rightarrow 1$

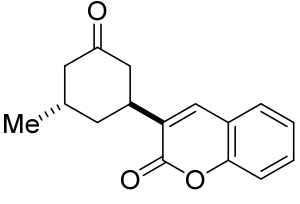

$\longrightarrow$ (1) ।

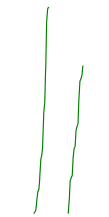

(d.r. $=13: 1)$

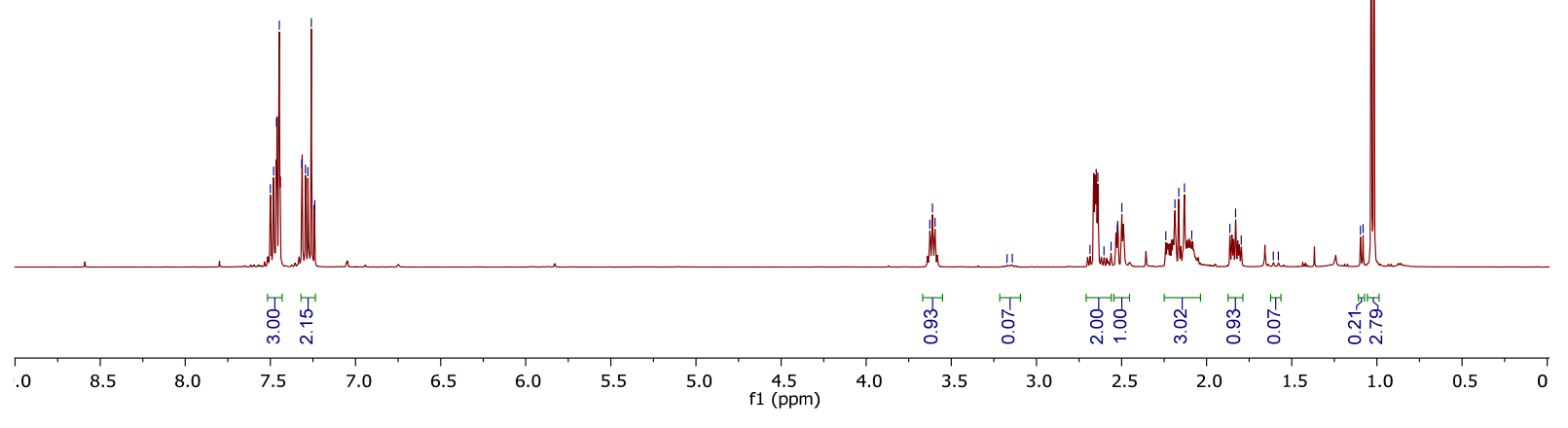

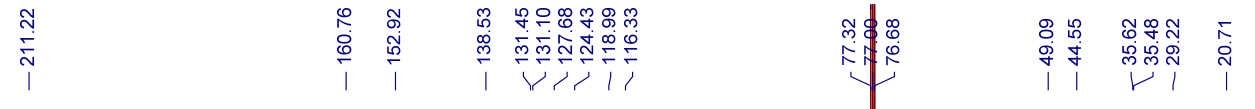<smiles>C[C@H]1CC(=O)C[C@H](c2cc3ccccc3oc2=O)C1</smiles>

(d.r. $=13: 1)$

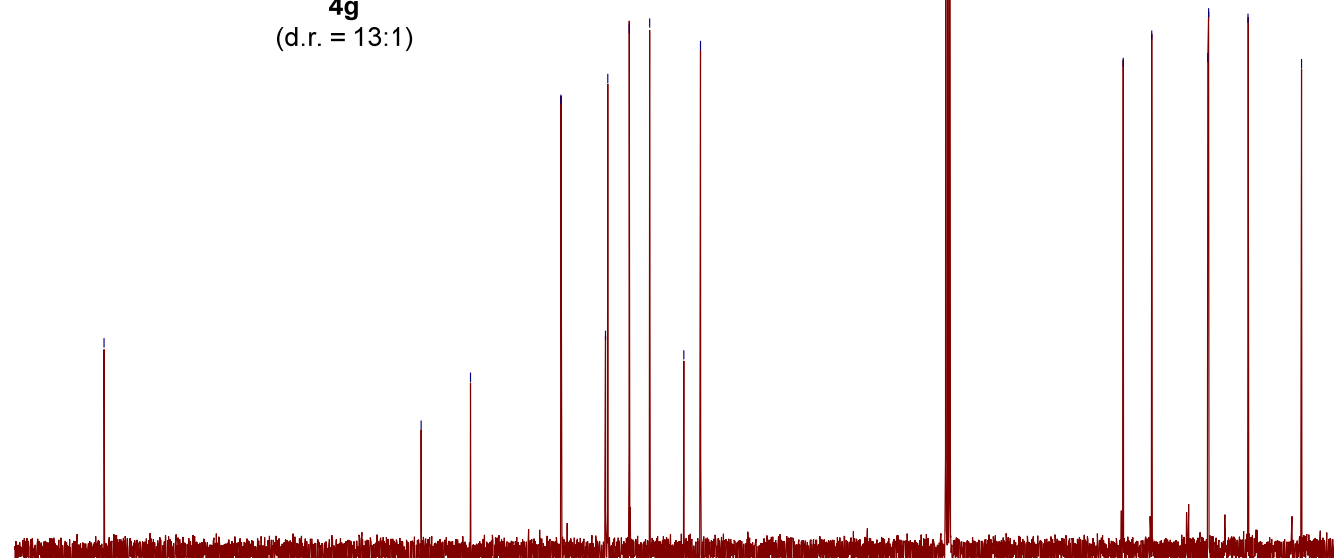

$\begin{array}{llllllllllllll}220 & 210 & 200 & 190 & 180 & 170 & 160 & 150 & 140 & 130 & 120 & 110 & 100 & 90\end{array}$

80

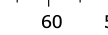

$50 \quad 40$

$30 \quad 20 \quad 10 \quad 0 \quad-10 \quad-20$ 


\section{COSY of $4 \mathrm{~g}$}

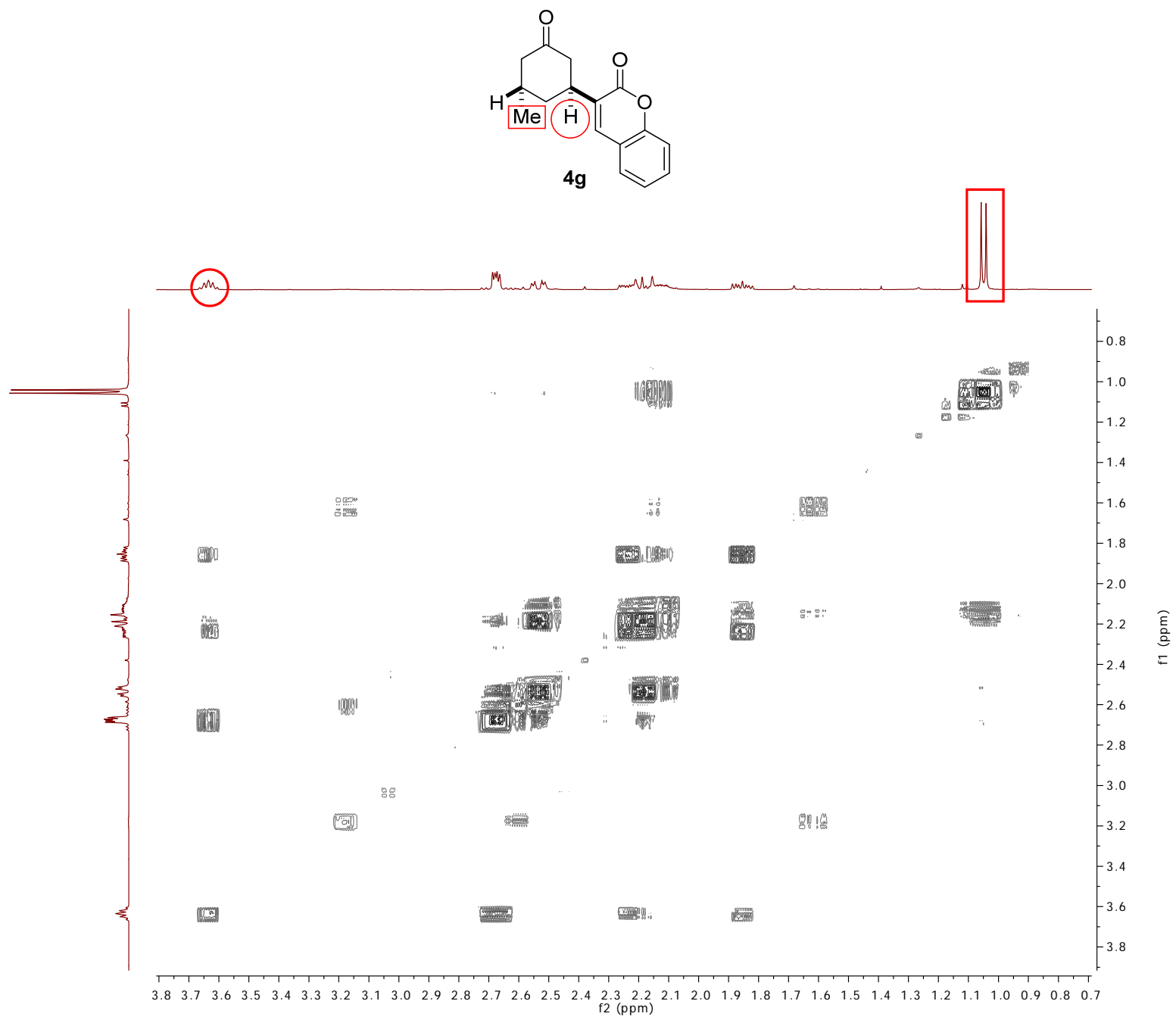




\section{NOESY of $4 \mathrm{~g}$}

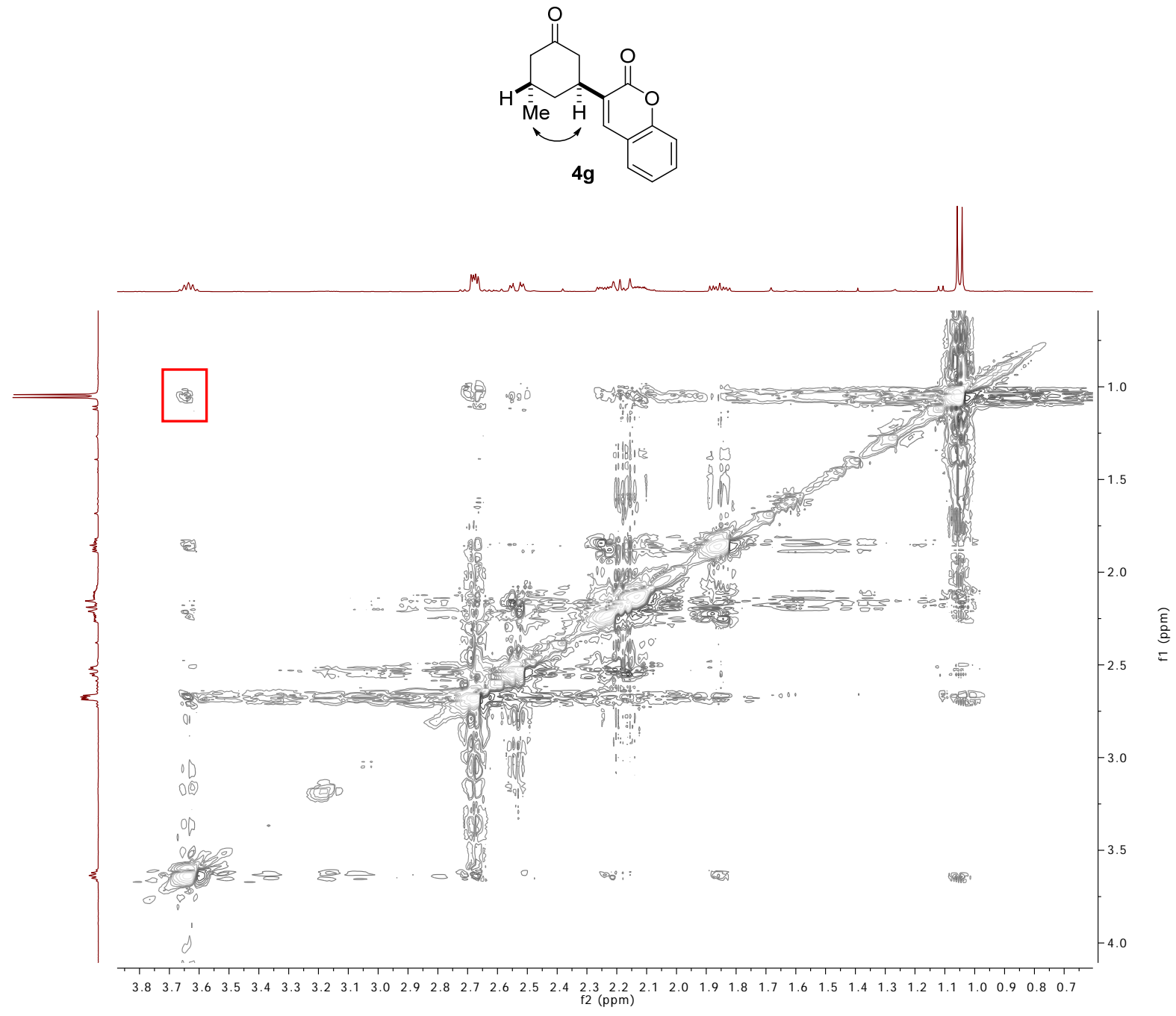




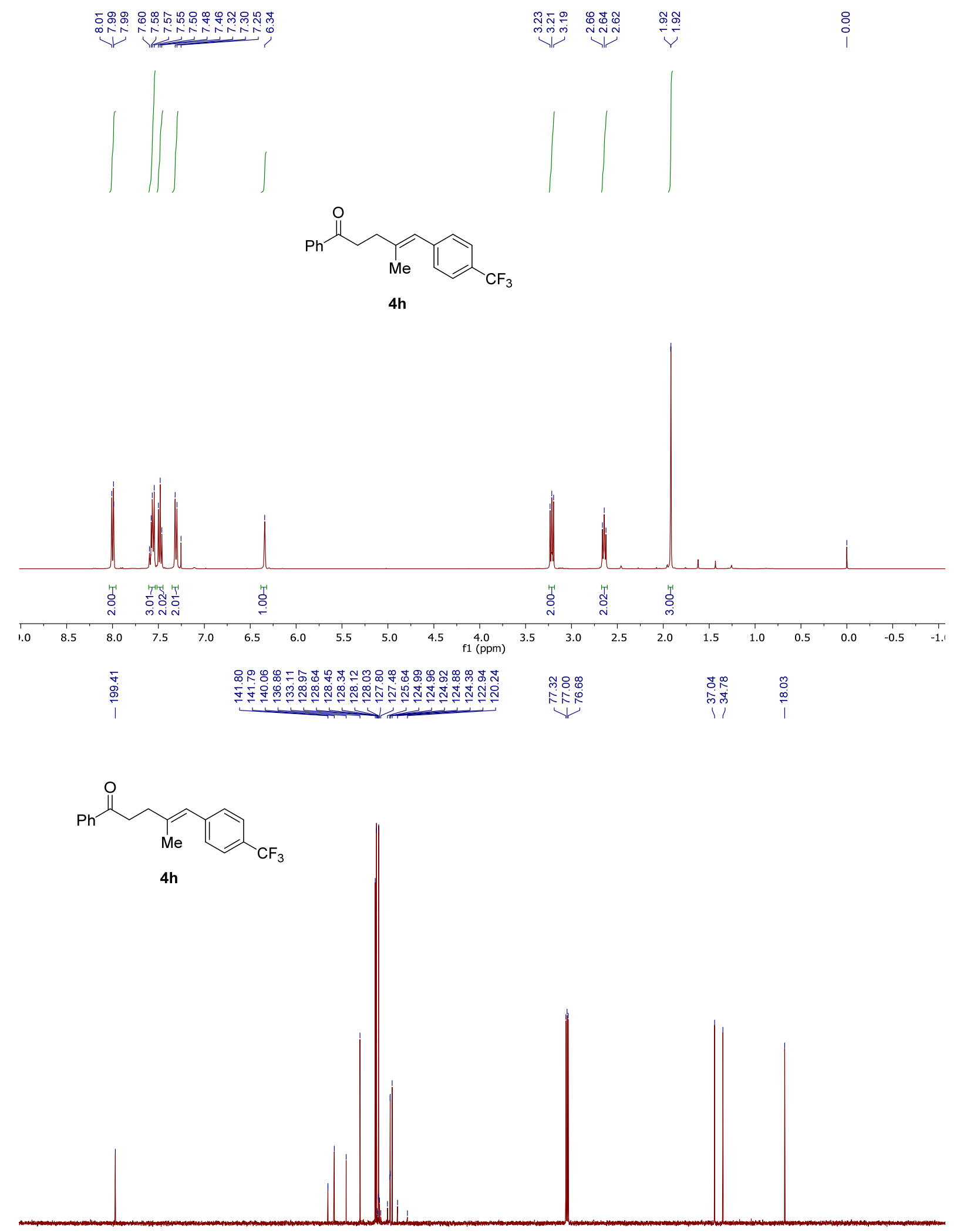

$\begin{array}{lllllllllllllllllllllllll} & 1 \\ 220 & 210 & 200 & 190 & 180 & 170 & 160 & 150 & 140 & 130 & 120 & 110 & 100 & 90 & 80 & 70 & 60 & 50 & 40 & 30 & 20 & 10 & 0 & -10 & -20\end{array}$ 


\section{${ }^{19}$ F NMR of $4 \mathrm{~h}$}

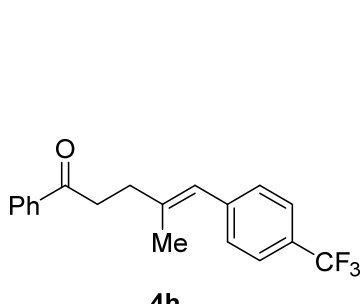

$\stackrel{\substack{0 \\ i}}{i}$

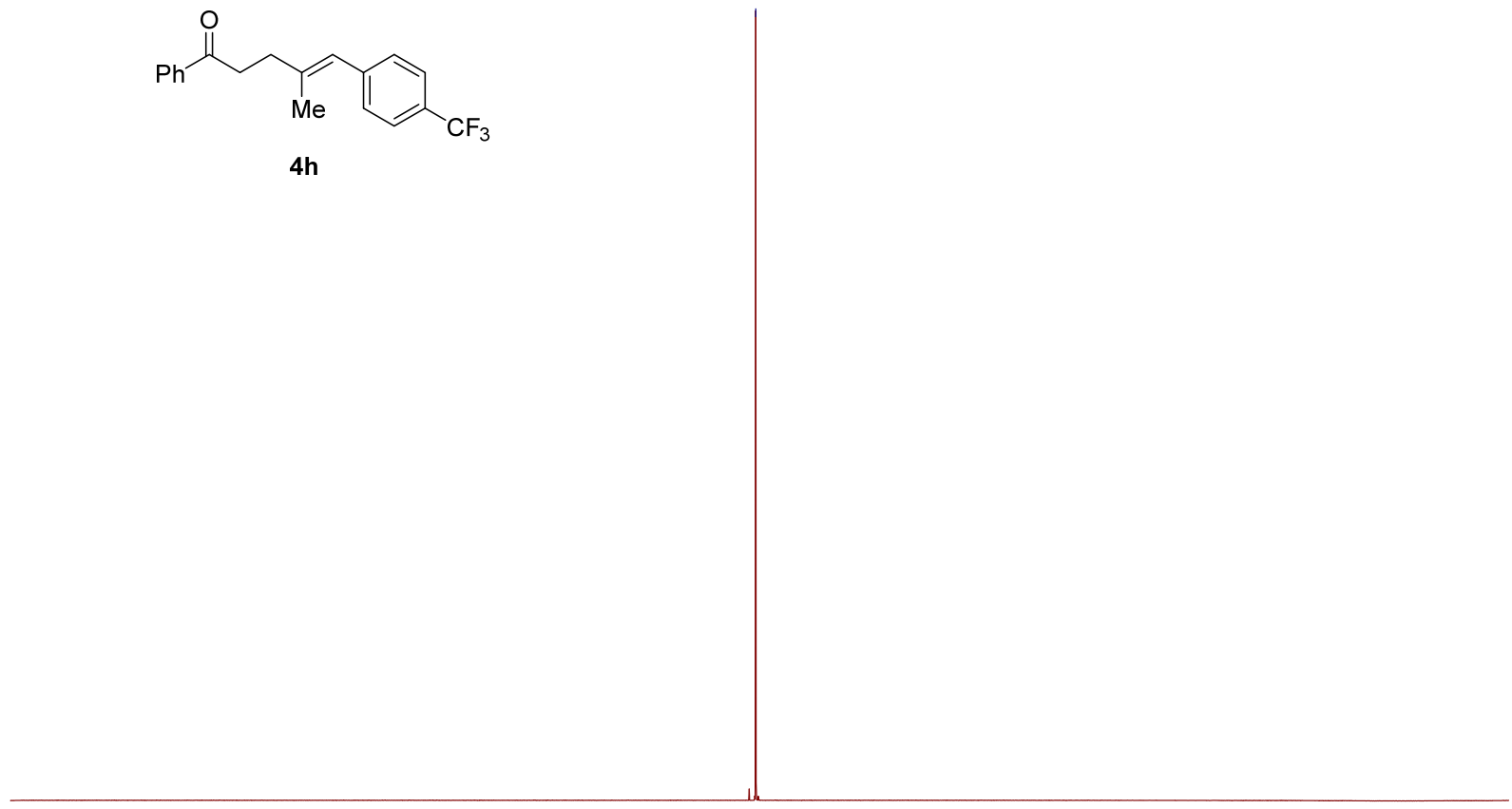

\begin{tabular}{lllllllllllllllllllllllllll}
\hline 60 & 50 & 40 & 30 & 20 & 10 & 0 & -10 & -20 & -30 & -40 & -50 & -60 & -70 & -80 & -90 & -100 & -110 & -120 & -130 & -140 & -150 & -160 & -170 & -180
\end{tabular} 

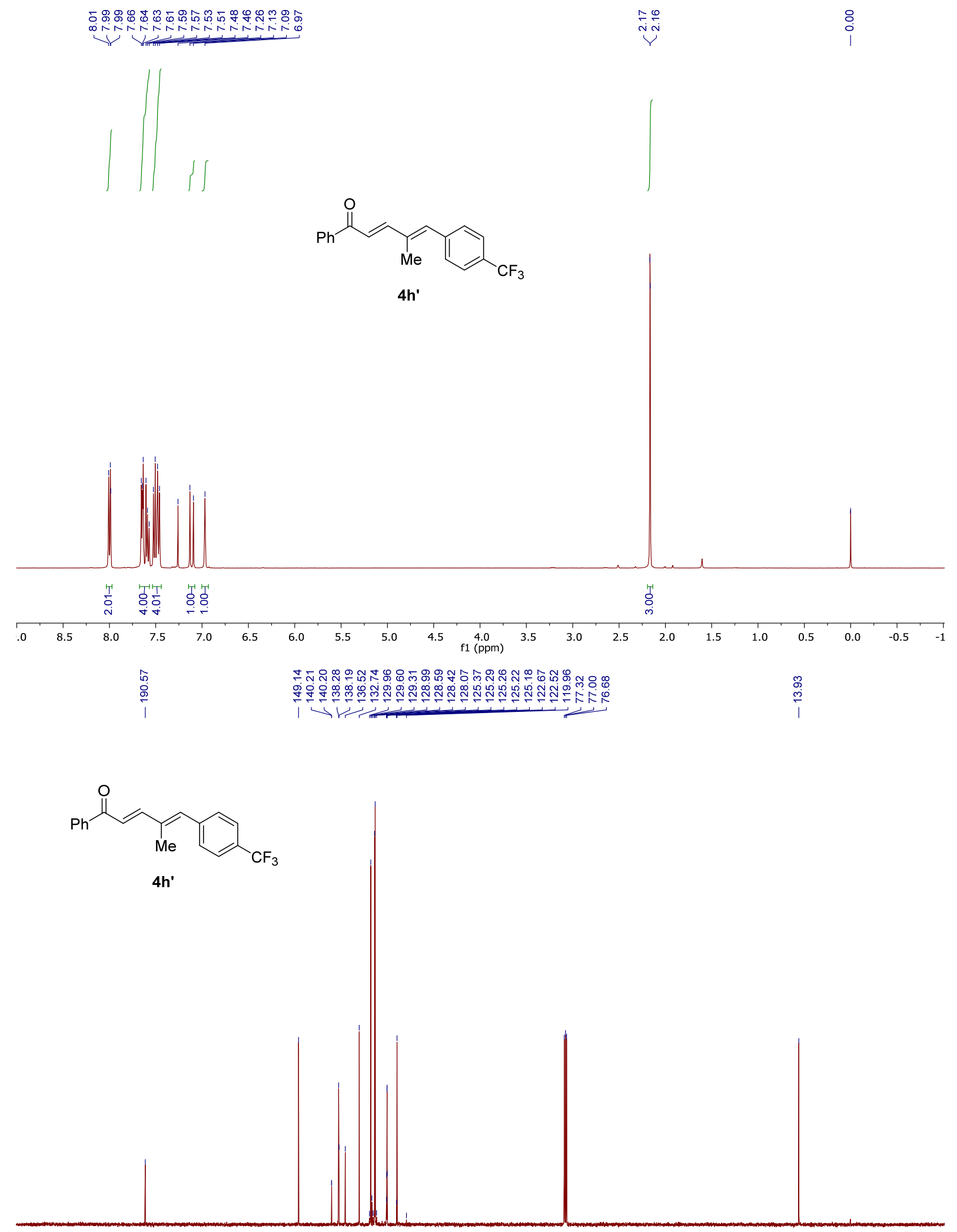

$\begin{array}{lllllllllllllllllllllllllllllllllllll}220 & 210 & 200 & 190 & 180 & 170 & 160 & 150 & 140 & 130 & 120 & 110 & 100 & 90 & 80 & 70 & 60 & 50 & 40 & 30 & 20 & 10 & 0 & -10 & -20\end{array}$ 


\section{${ }^{19}$ F NMR of $4 h$ '}

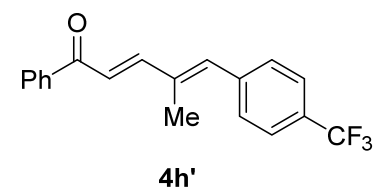

$\begin{array}{llllllllllllllllllllllllll}60 & 50 & 40 & 30 & 20 & 10 & 0 & -10 & -20 & -30 & -40 & -50 & -60 & -70 & -80 & -90 & -100 & -110 & -120 & -130 & -140 & -150 & -160 & -170 & -180\end{array}$ 

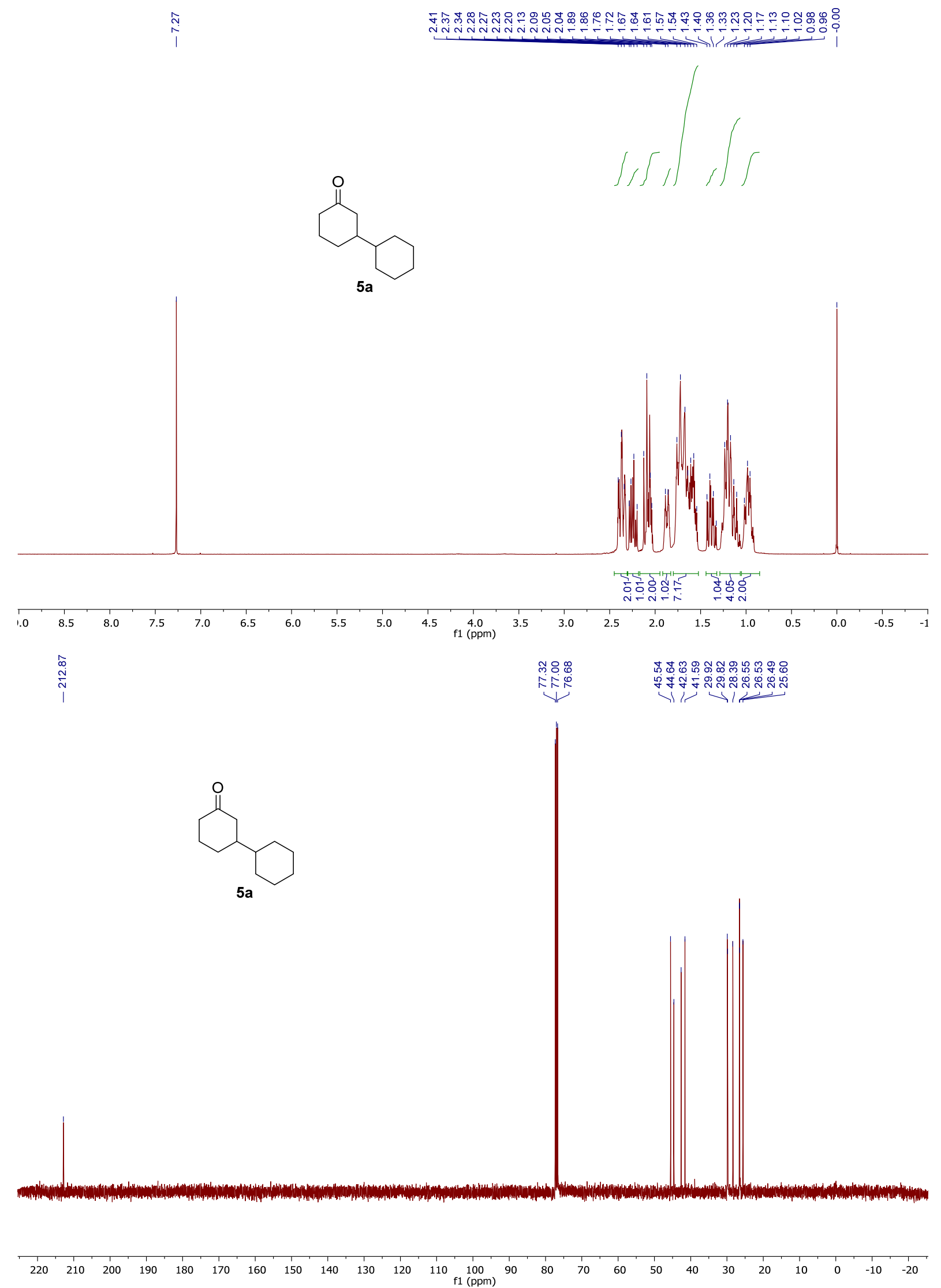

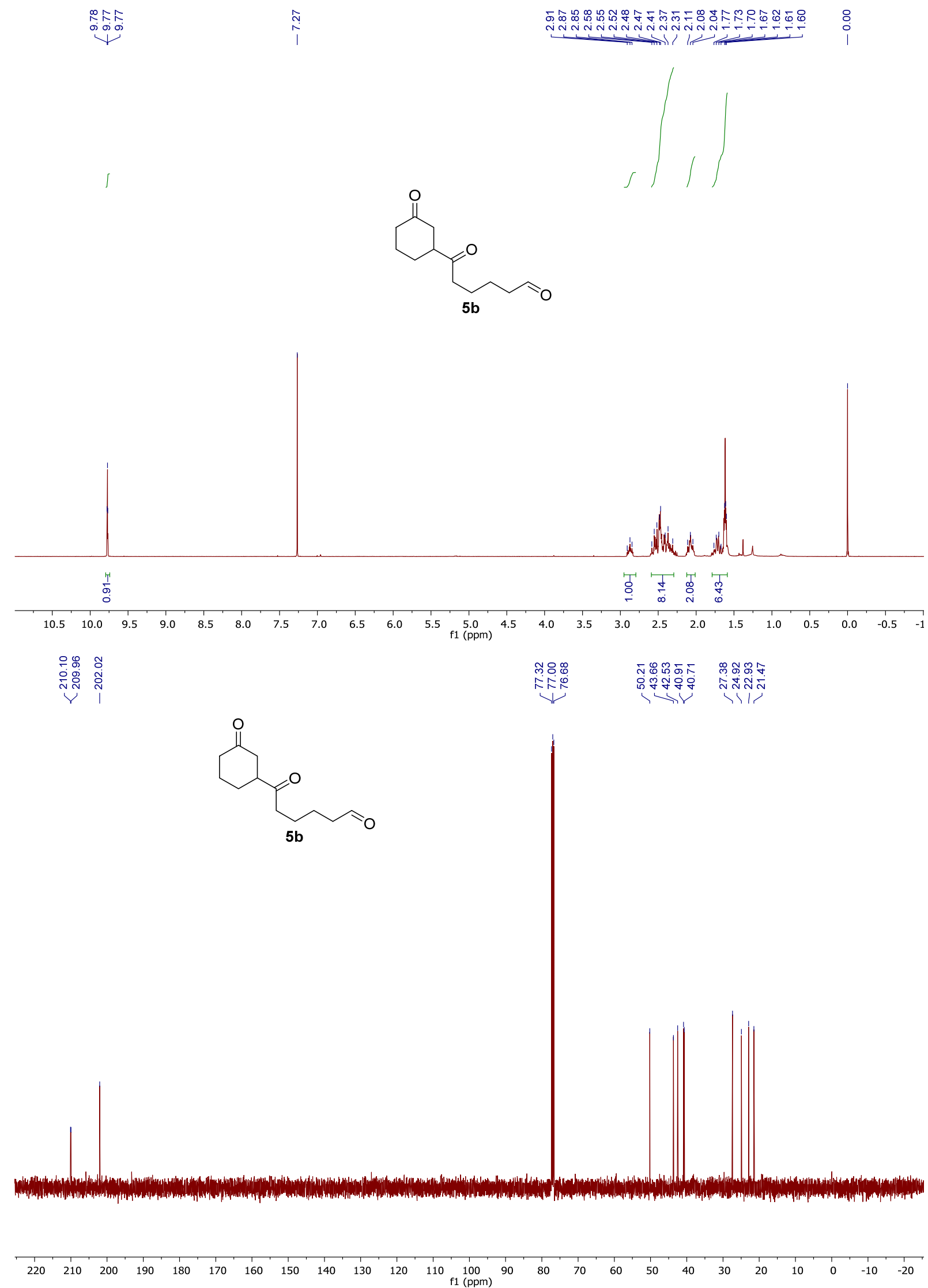


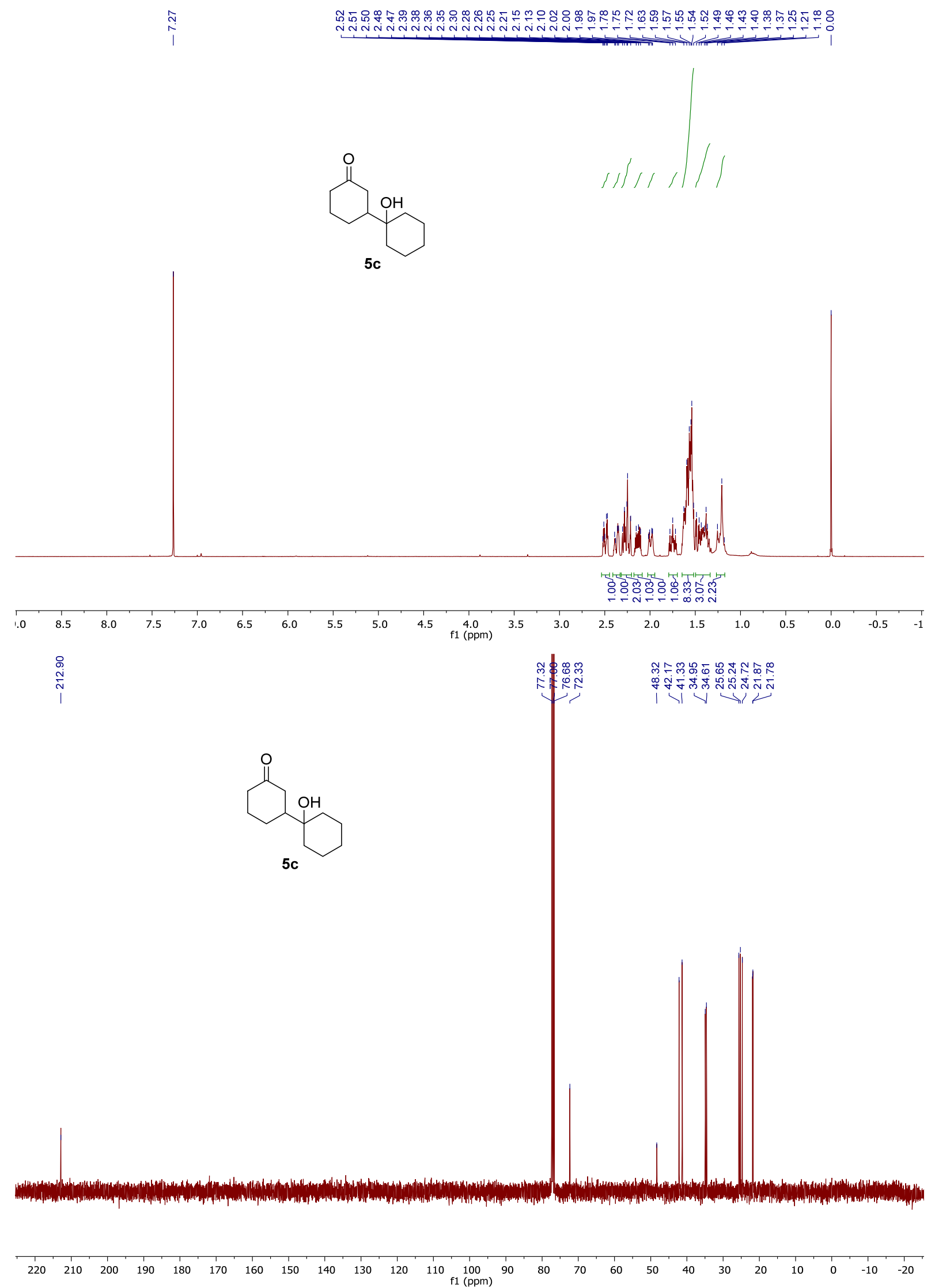



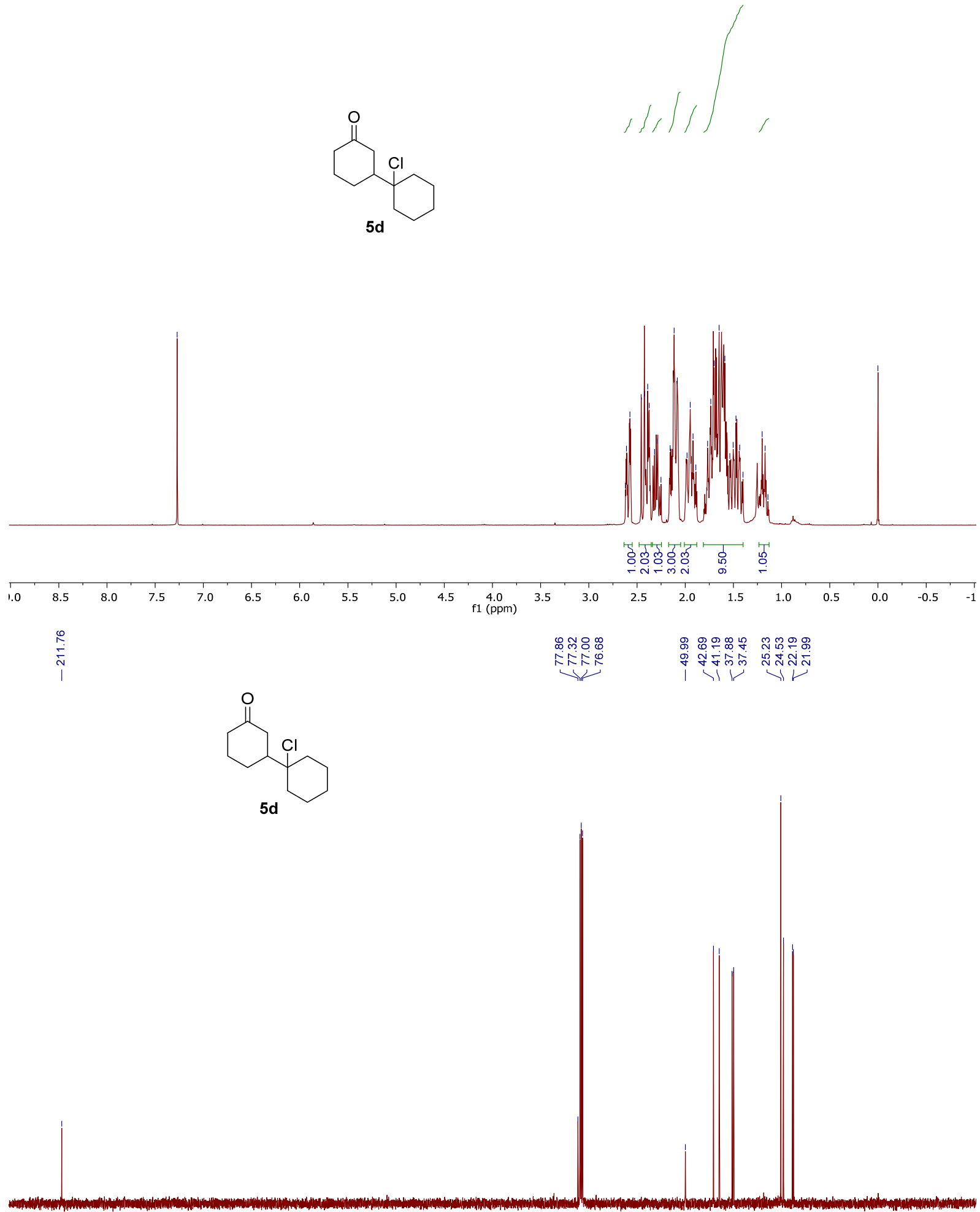

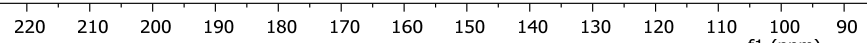

$\mathrm{f}_{1}(\mathrm{ppm})$

80

70

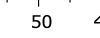

30

$\begin{array}{lllll}1 & 10 & 0 & -10 & -20\end{array}$ 


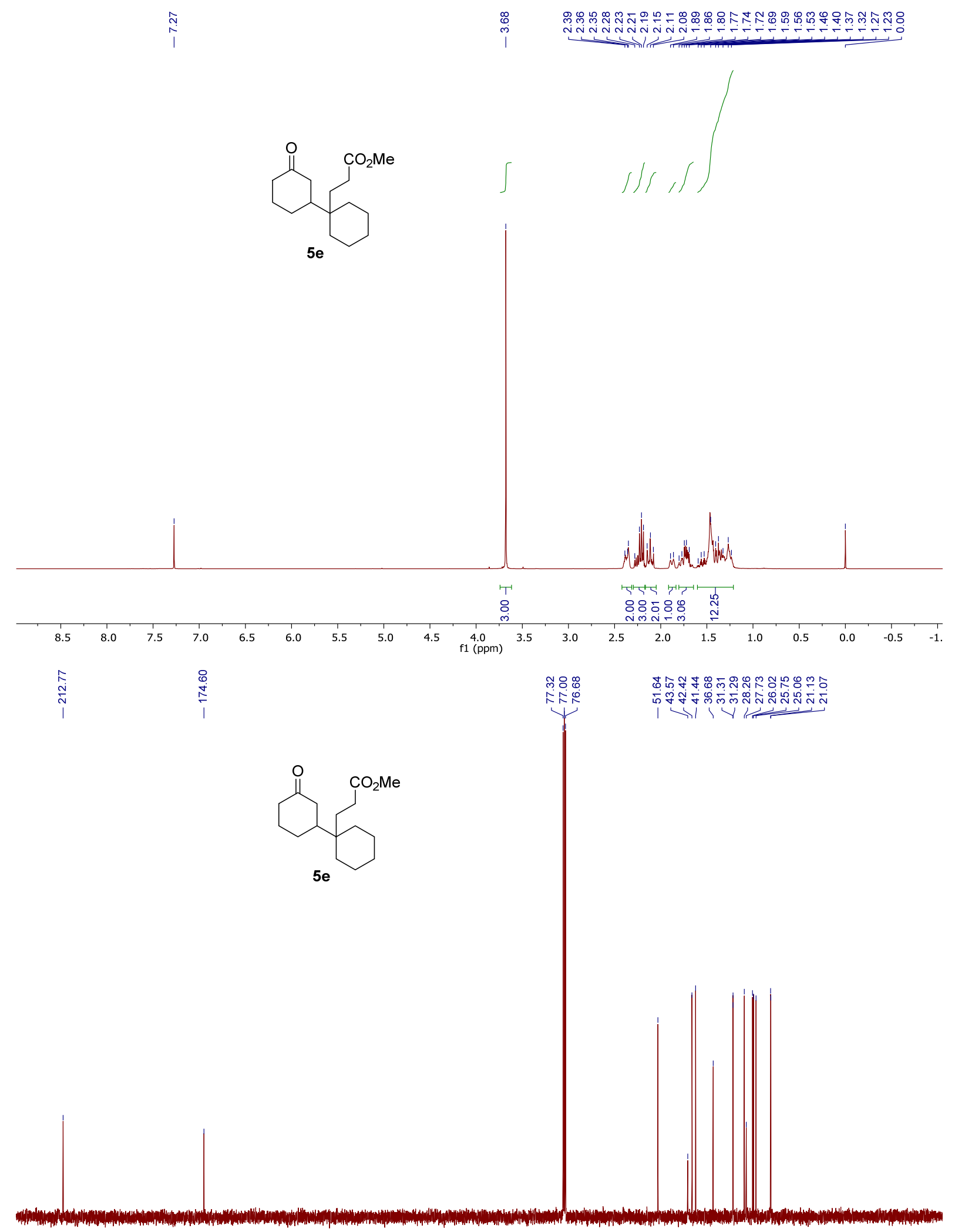

$\begin{array}{llllllllllllllllllllllllllllllll}220 & 210 & 200 & 190 & 180 & 170 & 160 & 150 & 140 & 130 & 120 & 110 & 100 & 90 & 80 & 70 & 60 & 50 & 40 & 30 & 20 & 10 & 0 & -10 & -20\end{array}$ 


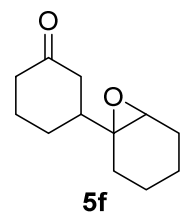

(d.r. $=1: 1)$

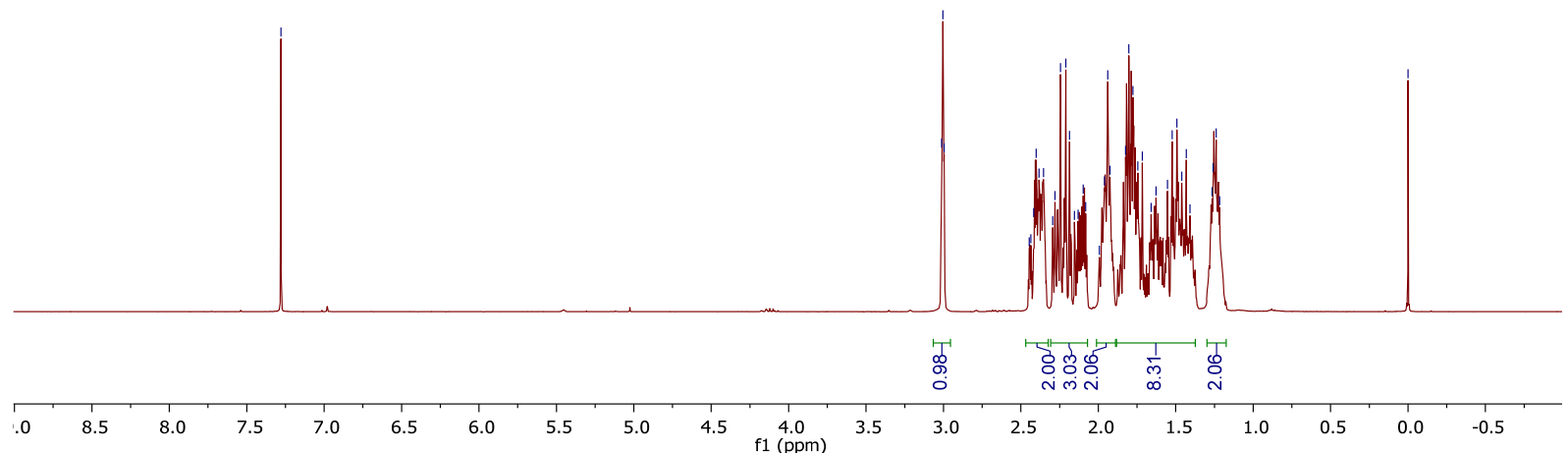

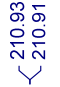

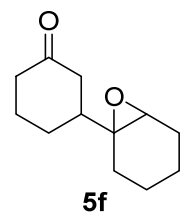

(d.r. $=1: 1)$

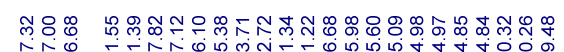

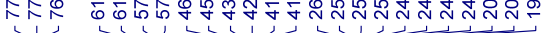

i.

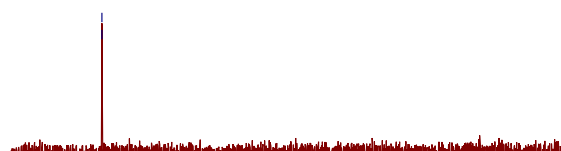

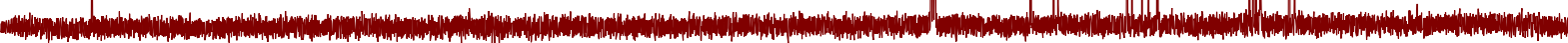

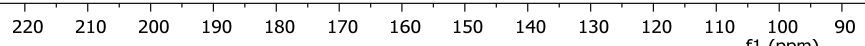

$f_{1}(\mathrm{ppm})$

80

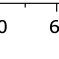

50

$-20$ 